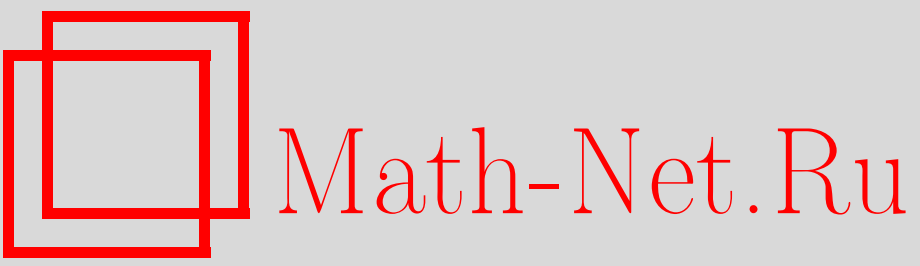

П. М. Ахметьев, Вложения компактов, стабильные гомотопические группы сфер и теория особенностей, УМH, 2000, том 55, выпуск 3, 3-62

DOI: https://doi.org/10.4213/rm290

Использование Общероссийского математического портала Math-Net.Ru подразумевает, что вы прочитали и согласны с пользовательским соглашением

http://www.mathnet.ru/rus/agreement

Параметры загрузки:

IP: 3.91 .87 .62

26 апреля 2023 г., 15:56:57 


\title{
ВЛОЖЕНИЯ КОМПАКТОВ, СТАБИЛЬНЫЕ ГОМОТОПИЧЕСКИЕ ГРУППЫ СФЕР И ТЕОРИЯ ОСОБЕННОСТЕЙ
}

\author{
П. М. Ахметьев
}

В обзоре рассмотрены новые приложения теории гомотопий и теории особенностей в геометрической и дифференциальной топологии. Получено решение проблемы Р. Давермана о вложении сфероподобного компакта в евклидово пространство. Найдены приложения в теории псевдоизотопий и топологии многообразий малых размерностей.

Библиография: 88 названий.

\section{СОДЕРЖАНИЕ}

Часть 1. Метод ортогонального проектирования в геометрической то-

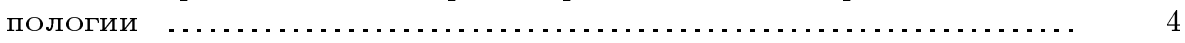

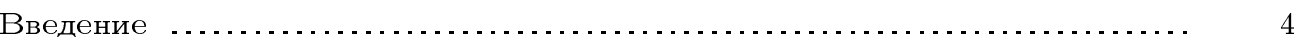

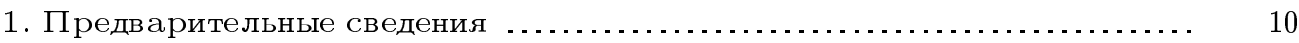

2. Дискретная реализация отображений $n$-мерной сферы $S^{n} \rightarrow S^{n}$ в

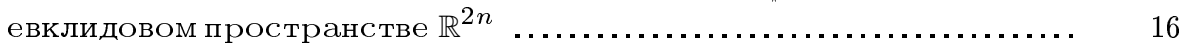

3. Дискретная реализация отображения $n$-мерной сферы $S^{n} \rightarrow S^{n}$ в

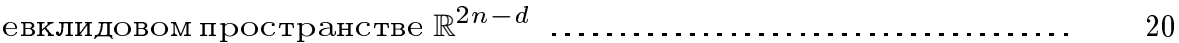

4. Вложение $S^{n}$-подобных компактов в евклидовы пространства $\quad . . . . . .22$

Часть 2. Метод ортогонального проектирования в дифференциальной

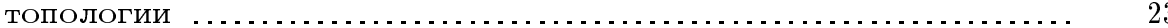

Введение . . . . . . 23

5. Формулы особенностей $I$-структуры погруженной 2-поверхности в $\mathbb{R}^{3} \quad 28$

6. Особенности $I$-структуры погруженного 3 -многообразия в $\mathbb{R}^{4} \quad \ldots \ldots . . \quad 37$

7. Диаграмма Серфа двупараметрического семейства функций $\ldots . . . . . .442$

8. Доказательство основной теоремы 3 . . . . . . . . . . . . . . . . . . . . . . . 46

9. Аппроксимация вложениями отображений общего положения поверхностей в объемлюшем пространстве $\mathbb{R}^{4}$. Теорема Рохлина о сигнатуре и аналоги инварианта странности для диаграммы узла .. 52

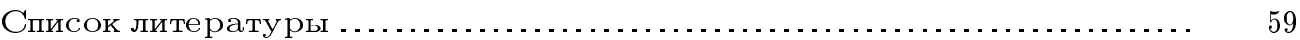

Работа выполнена при частичной финансовой поддержке Российского фонда фундаментальных исследований (грант № 99-01-00009). 
В философской работе А. Пуанкаре “Ценность науки” [62; с. 188] находим следующее. “Для того чтобы получить математическую непрерывность $n$-измерений, достаточно взять $n$ подобных шкал, ступени которых будут соответствовать различным значениям $n$ независимых величин, называемых координатами. Таким образом, получится изображение физической непрерьвности $n$ измерений ... " Далее в этой работе находим рассуждения, объясняюшие понятие размерности наблюдаемого пространства через мускульные ощушения и проекцию на сетчатку глаза.

Результаты, полученные в статье, относятся к различным разделам топологии. Тем не менее, все они объединены обшей идеей: при изучении фигуры $K \subset \mathbb{R}^{n+1}$ $(n+1)$-измерения, например, $n+1=4$, мы сможем упростить задачу и перейти к изучению фигуры $n$ измерений посредством анализа особенностей ортогонального проектирования $K \subset \mathbb{R}^{n+1} \rightarrow \mathbb{R}^{n}$. В рамках этой идеи будут получены новые приложения теории гомотопических групп сфер и теории особенностей в геометрической и дифференциальной топологии. Работа разбита на две части, каждая из которых снабжена введением, материал в каждой части излагается независимо.

В ч. 1 изучаются конечномерные компакты, имеющие “сложное" локальное строение, посредством более тонкого, по сравнению с размерностью компакта, топологического инварианта, а именно, минимальной размерности евклидова пространства, в которое данный компакт допускает вложение. В частности, получено положительное решение проблемы Р. Давермана о вложении сфероподобного $n$-мерного компакта, $n \neq 1,2,3,7$, в евклидово $2 n$-пространство (и частичные результаты в направлении ее обобшения). Новое в решении связано с приложением теории стабильных гомотопических групп сфер в рамках известного спектрального критерия вложимости.

В ч. 2 изучается пространство псевдоизотопий гладкого замкнутого (односвязного) многообразия высокой размерности. Определяется новая разновидность пространства функций с умеренными особенностями, которое доставляет важную информацию об основном пространстве псевдоизотопий. При изучении гомотопических свойств построенного пространства используются алгебраическая К-теория, классификационные теоремы теории особенностей и классификация погружений многообразий малых размерностей с точностью до кобордизма. Последний раздел посвящен приложениям в топологии гладких многообразий размерностей 3 и 4.

Работа состоит из 9 разделов, причем главные результаты работы, теоремы 1-3 и предложения 1,2 , имеют независимую нумерацию. Следствия и леммы нумеруются согласованно с разделами.

Написанию работы помогли обсуждения с М. М. Постниковым, Е. В. Щепиным и В. А. Васильевым. Полезньми оказались обсуждения с Н.Б. Бродским, В. М. Бухштабером, А. Ю. Воловиковым, А. В. Пажитновым, П. Е. Пушкарем, А. Б. Скопенковым, М. В. Смуровым, Ю. П. Соловьевым, А. Сючем, А. Ф. Харшиладзе, А. В. Хохловьм, А. В. Чернавским и П. Дж. Экклзомб которым я искренне благодарен.

\section{Часть 1. Метод ортогонального проектирования в геометрической топологии}

\section{Введение}

Одним из основных понятий, характеризуюших топологическое пространство, является понятие размерности. Это понятие было рассмотрено в работах А. Пуанка- 
ре и впервые строго определяется в работах П. С. Урысона. Различные подходы при определении понятия размерности связаны с вычислением кратности покрытия, задачей продолжения отображений, понятием гомологии циклов с различными областями коэффициентов. Один из наиболее простых и геометрических подходов предложен в работе П. С. Александрова [14] и связан с понятием $\varepsilon$-сдвига компакта на конечномерный полиэдр. Скажем, что метрический компакт $X$ имеет размерность, не превышающую $n$, если $\forall \varepsilon>0$ сушествует $\varepsilon$-отображение $f: X \rightarrow P^{n}$ компакта $X$ на $n$-мерньй полиэдр $P^{n}$, т.е. такое отображение, для которого $\operatorname{diam}\left(f^{-1}(p)\right)<\varepsilon, p \in P$. Ключевое свойство компактов размерности $\leqslant n$ выявляет следующая теорема, независимо доказанная Л. С. Понтрягиным [64] и Нёбелингом [61].

ТЕОРема ВЛОЖимости. Пусть $X-$ компакт размерности $\leqslant n$. В пространстве $C\left(X ; \mathbb{R}^{m}\right)$ непрерывных отображений этого компакта в $\mathbb{R}^{m}$ подпространство всех вложений образует открытое и всюду плотное подмножество при условии $m \geqslant 2 n+1$.

В самое последнее время рядом авторов [22], [48], [79] независимо были получены сильные обобшения теоремы Понтрягина-Нёбелинга. Укажем лишш на одно такое обобщение. Известно, что для компактов общего вида логарифмический закон сложения размерности имеет следующий вид $\operatorname{dim}(X \times X) \leqslant 2 \operatorname{dim}(X)$. Если для $n$-мерного компакта вьполнено строгое неравенство $\operatorname{dim}(X \times X)<2 n$, то для такого компакта пространство $E\left(X, \mathbb{R}^{2 n}\right)$ вложений является всюду плотньм подмножеством в пространстве $C\left(X, \mathbb{R}^{2 n}\right)$ всех непрерывных отображений. В настоящей работе теорема Понтрягина-Нёбелинга обобшается с другой точки зрения, что приводит к неожиданным приложениям стабильной теории гомотопий сфер. Нами рассматривается случай $m \leqslant 2 n+1$. Накладывая те или иные ограничения на топологию пространства $X$, pacсмотрим проблему нахождения минимальной размерности евклидова пространства, в которое компакт $X$ допускает вложение.

ОПРЕДЕЛЕНИЕ. Скажем, что метрический компакт $X$ является $S^{n}$-подобным, если $\forall \varepsilon>0$ сушествует $\varepsilon$-отображение $f: X \rightarrow S^{n}$ компакта на сферу размерности $n$.

ПРОБЛЕМА ДАВЕРМАНА О ВЛОЖЕНИИ $S^{n}$-ПОДОБНОГО КОМПАКТА [21; Проблема Е16]. Верно ли, что произвольный $S^{n}$-подобный компакт можно вложить в пространство $\mathbb{R}^{2 n}$ ?

Понятию $S^{n}$-подобного компакта можно дать эквивалентное определение в терминах обратного предела спектра отображений $n$-мерной сферы. Понятие спектра мы здесь рассматриваем, как в [37]. Это определение напоминается в разделе 4. Пусть $X$ - обратный предел этого спектра, снабженный стандартной метрикой. Произвольный $S^{n}$-подобньй компакт, в смысле предшествующего определения, гомеоморфен пространству $X$ для некоторого выбора спектра.

Главным результатом первой части работы является следующая теорема.

Теорема 1. B предположсении $n \neq 1,2,3,7$ произвольный $S^{n}$-подобныи компакт допускает вложение в пространство $\mathbb{R}^{2 n}$.

Близкий по формулировке результат другим методом получен в работе [45]. Там доказано, что произвольный $T^{n}$-подобный компакт, т.е. компакт, допускающий сколь 
угодно мальй $\varepsilon$-сдвиг на $n$-мерньй тор $T^{n}$, при условии $n \neq 1$ вкладывается в пространство $\mathbb{R}^{2 n}$. При $n=1$ проблема Давермана имеет отрицательное решение. Действительно, для произвольного натурального $p>1$ определено пространство $p$-адического соленоида, которое является косым произведением канторовского совершенного множества на окружность. Напомним, что это пространство получено в результате пересечения бесконечной последовательности вложенных полноториев, каждый из которых вложен в предыдуший в окрестности его средней линии со степенњ $p$, при этом диаметры полноториев стремятся к 0. Это пространство имеет размерность 1 , тем не менее не может быть вложено в плоскость. Можно ли обобшить понятие соленоида на старшие размерности? На основании предложения 1.Б (формулировку см. далее) выдвигается следующая гипотеза.

ГИПотЕЗА. Существуют $S^{3}-u S^{7}$-подобные компакты, которые не вкладьваются в евклидовы пространства размерностей 6 и 14 соответственно.

В случае положительного решения наиболее интересной представляется проблема явного описания таких компактов, обладающих богатой структурой симметрии.

Следуюшая проблема является естественным обобшением проблемы Давермана.

ОБОБЩЕННАЯ ПРОБЛЕМА ДАВЕРМАНА О ВЛОЖЕНИИ $S^{n}$-ПОДОБНОГО КОМПАКТА. Для данного натурального п найти наименьшую размерность $m=m(n)$ евклидова пространства $\mathbb{R}^{m}$, в которое вкладывается произвольный $S^{n}$-подобный компакт.

Автору не известно, верно ли, что функция $\bar{M}(n)=\min _{n^{\prime}>n}\left(2 n^{\prime}-m\left(n^{\prime}\right)\right)$ такова, что $\lim _{n \rightarrow \infty} \bar{M}(n)=+\infty$. В направлении решения обобшенной проблемы Давермана доказан следующий результат.

ТЕОРема 2. Для произвольного сколь угодно большого числа $k$ существует число $n=n(k)$ такое, что произвольныи $S^{n}$-подобный компакт вкладывается в пространство $\mathbb{R}^{2 n-k}$. Эквивалентно, $\sup _{n}(2 n-m(n))=+\infty$.

Решение проблем Давермана можно условно разбить на две части. Первая часть связана с геометрическими конструкциями, возникающими в процессе представления компакта как обратного предела спектра. В книге [37] доказано, что произвольньй $n$-мерный компакт $X$ допускает представление как обратный предел спектра, который состоит из отображений полиэдров размерности $n$. При этом $S^{n}$-подобньй компакт представляется обратным пределом спектра отображений $n$-мерной сферы. Критерий вложения обратного предела спектра, которьй мы используем, является прямым обобщением результатов независимых работ многих авторов, см., например, [73], [75].

Вторая часть решения проблемы связана с так назьваемой проблемой реализации отображений, которая в интересуюшем нас случае связана с вычислением и описанием гомотопических групп сфер. Результаты выгислений в некоторых случаях могут быть использованы для построения вложений конкретных компактов. Решение проблемы Давермана вытекает из положительного решения гипотезы Хопфа, полученного Адамсом. Решение обобшенной проблемы Давермана следует искать, исследуя обобщенный инвариант Хопфа и связанную с этим инвариантом ЕНР-последовательность Джеймса-Уайтхеда [41], геометрическое построение которой предложено в работе [46]. Сформулируем понятие реализации отображений как основной инструмент в решении задачи вложения компактов. 
ОПРЕДЕЛЕНИЕ ПОНЯТИЯ ДИСКРЕТНОЙ РЕАЛИЗАцИИ. Пусть $A, B, X$ - глаДКИе ( $A, B$ - замкнутые, если не оговорено противное) многообразия, $\operatorname{dim}(A)<\operatorname{dim}(X)$, $\operatorname{dim}(B)<\operatorname{dim}(X), f: A \rightarrow B$ - непрерьвное отображение и $I: B \subset X$ - гладкое вложение. Скажем, что отображение $f$ дискретно реализуется в $X(\operatorname{rel} I)$ (т.е. относительно $I$ ), если существует последовательность вложений $\left\{h_{n}\right\}, h_{n}: A \rightarrow X$, такая, что выполнено условие

$$
\lim _{n \rightarrow \infty} \sup _{x \in A}\left\|h_{n}(x)-I \circ f(x)\right\|=0 .
$$

Эквивалентно, отображение $f$ дискретно реализуется в $X(\mathrm{rel} I)$, если существует вложение $\bar{h}: A \rightarrow X$, сколь угодно близко аппроксимирующее композицию $I \circ f$ в $C^{0}$-метрике пространства отображений. Допуская вольность речи, будем говорить, что $f$ дискретно реализуется в $X$ (или реализуется в $X$ ), предполагая при этом, что $I$ задано. В интересующем нас случае $A, B$ суть $n$-мерные сферы, $X=\mathbb{R}^{m}$ - евклидово пространство размерности $m, I$ - стандартное вложение.

Близкое понятие изотопической реализации отображений было изучено Л. В. Келдьш в работе [44]. Строго говоря, мы рассматриваем аналогичное понятие, связанное с переходом от объемлемой изотопии пространства-образа к более часто используемому понятию изотопии вложения, а также с переходом из топологической категории в гладкую категорию. Понятие изотопической реализации используется в работе [73] для более тонкого анализа, связанного со спектральным критерием вложимости в случае, когда этажи обратного предела, определяюшие компакт, не являются многообразиями. Это же понятие является основным при определении понятия "ручного" и “дикого" вложения компактов в теории М. А. Штанько, см. [74].

ОПРЕДЕЛЕНИЕ ПОНЯТИЯ ИЗОТОПИЧЕСКОЙ РЕАЛИЗАЦИИ. СКажеМ, чТо ОТображение $f$ изотопически реализуется в $X(\mathrm{rel} I)$, если существует $C^{\infty}$-гладкая гомотопия (псевдоизотопия) $\left.\left.F_{t}: A \rightarrow X, t \in\right] 0 ; 1\right]$, удовлетворяющая следующим условияM.

1. Для каждого $t \in] 0 ; 1]$ отображение $F_{t}: A \rightarrow X$ является гладким вложением.

2. Гомотопия $F_{t}$ продолжается при $t \rightarrow 0+$ до непрерьвного отображения $I \circ f=$ $F_{0}: A \rightarrow X$.

Понятие изотопической реализации важно со многих точек зрения. Во-первых, на основании этого понятия в [73] доказаны не только достаточные, но и необходимые условия вложимости компактов. Во-вторых, уже в простейшем случае, когда $A, B$ являются $n$-сферами, проблема изотопической реализации приводит к нерешенным задачам в топологии контролируемых отображений. Очевидно, что изотопическая реализация отображения $f$ влечет его дискретную реализацию. Верно ли обратное?

В работе [56] доказано, что в случае, когда $f$ - кусочно-линейное отображение коразмерности $>2$, обратное утверждение истинно. В работе автора [8] доказано, что при $n=4 k+1, n>5$, всякое отображение $f: S^{n} \rightarrow S^{n}$ изотопически реализуется в пространстве $\mathbb{R}^{2 n}$. Этот результат является технически сложным, кроме того, в дальнейшем планируется исследовать возможные обобшения и результат нельзя считать окончательным. С другой стороны, в [76] построен пример отображения $f: S^{1} \rightarrow \mathbb{R}^{3}$, 
параметризующего дикую дугу, допускающего дискретную реализацию, но не допускающего изотопическую. ${ }^{1}$

С.А. Мелихов недавно построил удивительньй пример отображения $f: D^{3} \cup P^{3} \rightarrow$ $\mathbb{R}^{6}$, где $P^{3}$ - трехмерньй полноторий, $D^{3}$ - трехмерньй диск, $D^{3} \cup P^{3}$ - их несвязное объединение. Это отображение допускает дискретную реализацию, но не допускает изотопическую, см. [56]. (Для кусочно-линейных отображений, и даже для более шшрокого класса отображений, описанного в [56], понятия дискретной и изотопической реализации эквивалентны.) Легко этот пример обобщить и построить при каждом $n \geqslant 3$ отображение $f: S^{n} \rightarrow \mathbb{R}^{2 n}$, которое допускает дискретную реализацию, но не допускает изотопическую; это было сделано в совместной работе С. А. Мелихова и автора [11]. При условии метастабильного ранга в этой же работе удалось описать полное алгебраическое препятствие к изотопической реализации отображения $f$. В частности, из этого вытекает следующее утверждение.

ТЕОРЕМА ОБ ИЗОТОПИЧЕСКОЙ РЕАЛИЗАЦИИ В КОРАЗМЕРНОСТИ 1. При условии метастабильного ранга, т.е. при условии $m>\frac{3(n+1)}{2}$, для произвольного отобрахсения $f: S^{n} \rightarrow \mathbb{R}^{m}$, которое дискретно реализуется, отобрахсение $I \circ f: S^{n} \rightarrow \mathbb{R}^{m+1}, I: \mathbb{R}^{m} \subset \mathbb{R}^{m+1}$, реализуется изотопически.

Доказательство этого факта основано на достаточно трудоемком критерии, которьй построен в терминах изовариантных отображений в работе [56], по аналогии с критерием Хефлигера-Хирша изотопности двух гладких вложений, в форме, предложенной в книге [1].

Понятие изотопической реализации следует рассматривать в более общей категории. Пусть $E m b\left[A=S^{n} ; X=\mathbb{R}^{n+k}\right]$ - пространство $C^{0}$-гладких вложений $A \rightarrow X$, снабженное $C^{0}$-топологией. Рассмотрим пространство $W$, полученное пополнением пространства $E m b\left[S^{n} ; \mathbb{R}^{n+k}\right]$ в $C^{0}$-топологии пространства отображений. Точки из подпространства $W \backslash E m b\left[S^{n} ; \mathbb{R}^{n+k}\right]$, которое мы будем обозначать через $V$, представляются сингулярными непрерывными отображениями $f: S^{n} \rightarrow \mathbb{R}^{n+k}$, дискретно реализуюшимися в пространстве $\mathbb{R}^{n+k}$.

По аналогии с определением Квинна [67] (см. также [36]) определено пространство концов $e\left(E m b\left[S^{n} ; \mathbb{R}^{n+k}\right]\right)$ исходного пространства вложений, которое состоит из всевозможных отображений $w:\left[0 ; 1\left[\rightarrow E m b\left[S^{n} ; \mathbb{R}^{n+k}\right]\right.\right.$. Обозначим через $\bar{e} \subset e$ подпространство концов, допускающих непрерывную компактификацию $\bar{w}:[0 ; 1] \rightarrow W$. Определено отображение $F: \bar{e} \rightarrow W$ по формуле $F(\bar{w})=\bar{w}(1)$. Обозначим через $E$ образ пространства $\bar{e}$ при этом отображении.

Пространство концов $\bar{e}$ представляется теперь как пространство изотопических реализаций отображений из $E$. Проблема изотопической реализации может быть обобщена и переформулирована следующим образом: верно ли, что произвольной предельной точке $w_{0} \in W$ соответствует некоторьй конец в пространстве $\bar{e}$, иными словами, верно ли, что $E=W$ ? Конструкция Мелихова дает отрицательньй ответ на этот вопрос. Для отображений в метастабильном ранге пространство $W \backslash E$ оказьвается непустым. Каждая точка $W \backslash E$ принадлежит $V$, причем пространство $E m b\left[S^{n} ; \mathbb{R}^{n+k}\right]$ в точках из $W \backslash E$ не является локально-линейно-связным. Построенное обобщение

\footnotetext{
${ }^{1}$ Замечание при корректуре: Автору стало известно, что ранее этот пример был построен Л.В. Кнлдыш в работе "Псевдоизотопия некоторых локально-заузленных дуг в $\mathbb{E}^{3}$ ", Докл. АН CCCP. 1971. T. 200. №1. C. 21-23.
} 
в случае $A=X=S^{n}$ связано с проблемой аппроксимации клеточно-подобных отображений гомеоморфизмами. Напомню, что согласно теореме Чернавского [18] пространство Нотео $\left(S^{n} ; S^{n}\right)$ является локально-стятиваемым. Отсюда, как заметил С. А. Мелихов, легко вытекает, что проблема изотопической реализации для отображений $S^{n} \rightarrow S^{n}$ сфер (в сфере-образе) решается положительно, более того, сферу можно заменить на произвольное многообразие.

Теперь рассмотрим ряд простейших примеров, поясняющих понятие дискретной реализации. Очевидно, что любое отображение $A \rightarrow B$ при условии $\operatorname{dim}(A)=\operatorname{dim}(B)$ $=n$ реализуется в $(2 n+1)$-мерном многообразии $X$. Более тонкие рассуждения, приведенные в [75], показывают, что произвольное отображение $f: D^{n} \rightarrow D^{n}, D^{n}=$ $\left\{x \in \mathbb{R}^{n},\|x\|<1\right\}$, дискретно (в действительности, изотопически) реализуется относительно стандартного вложения $I: D^{n} \rightarrow D^{2 n}$. Кроме того, существует отображение $g: S^{2} \rightarrow S^{2}$, которое не реализуется относительно вложения $I: S^{2} \subset \mathbb{R}^{3}$. В качестве $g$ можно выбрать отображение, заданное в комплексных координатах на $S^{2}$ по формуле $z \rightarrow z^{2}$.

Основные теоремы 1,2 вытекают из нижеследующих предложений 1.А и 2. Предложения 1 и 2 являются важными теоремами в дифференциальной топологии. Тем не менее в рамках настояшей работы они имеют лиш вспомогательное значение.

ПРЕДЛОЖЕНИЕ 1. А. Произвольное отображение $f: S^{n} \rightarrow S^{n}$ в предположении $n \neq 7, n>4$ дискретно реализуется в пространстве $\mathbb{R}^{2 n}$.

Б. При $n=1,3,7$ существует отображсние $f: S^{n} \rightarrow S^{n}$, которое дискретно не реализуется в $\mathbb{R}^{2 n}$.

ПРЕДЛОЖЕНИЕ 2. Для произвольного натурального $d$ найдется такое число $n=n(d)$, ито любое отображение $f: S^{n} \rightarrow S^{n}$ дискретно реализуется в пространстве $\mathbb{R}^{2 n-d}$.

ЗАМЕчАнИЕ. Из теоремы об изотопической реализации в коразмерности 1 сразу вытекает, что предложение 2 справедливо и для случая изотопической реализации.

ЗАмечАниЕ. В предположении $m>\frac{3(n+1)}{2}$ из теоремы Хефлигера [34], [1] вытекает, что любые два вложения $S^{n} \subset \mathbb{R}^{m}$ связаны изотопией. Поэтому, не ограничивая обшности, можно считать, что в предложениях 1 и 2 говорится о реализации соответствуюшего отображения относительно стандартного вложения $S^{n} \subset \mathbb{R}^{m}$. Предложение 1.А было доказано в работе [8] в частном случае $n \neq 2^{l}-1$ и в работе [9] в общем случае. Краткое доказательство предложения 1.Б приводится в [8].

Теоремы 1 и 2 можно рассматривать как утверждения из теории особенностей. Рассмотрим динамическую систему $\left\{\alpha_{i}: S^{n} \rightarrow S^{n}\right\}$, заданную на сфере $S^{n}$. В важном частном случае $\alpha_{i}=\alpha$ не зависят от $i$. Пусть $X=\lim _{\leftarrow} \alpha_{i}$ - пространство траекторий динамической системы $\alpha_{i}$. Пространство $X$ совпадает с пространством обратного предела спектра $\left\{\alpha_{i}\right\}$ и к нему применимы теоремы 1 и 2 . Действительно, обобшенная проблема Давермана состоит в вычислении наименьшего числа координатных функций, определенных на пространстве траекторий $X$ и требуюшихся для полного разделения точек этого пространства.

Итак, доказательство основных теорем основано на новом приложении стабильной теории гомотопий и позволит по иному подойти к классическим проблемам вложения 
компактов в евклидово пространство. Укажем для примера на некоторые из них. Для данного компакта $X$ рассмотрим следуюшие условия.

(a) Существует $T$-эквивариантное отображение конфигурационных пространств:

$$
(X \times X \backslash \Delta ; T) \rightarrow\left(\mathbb{R}^{m} \times \mathbb{R}^{m} \backslash \Delta ; T\right) \approx\left(S^{m-1} ; T\right)
$$

Здесь $T$ - свободная инволюция на декартовом произведении пространств $X$ и $\mathbb{R}^{m}$ с удаленными диагоналями $\Delta$, которые называются "взрезанными квадратами" соответствующих пространств. Имеется естественное эквивариантное отображение $\left(S^{m-1} ; T\right) \rightarrow\left(\mathbb{R}^{m} \times \mathbb{R}^{m} \backslash \Delta ; T\right)$, которое является гомотопической эквивалентностью пространств с инволюциями, причем на $S^{m-1}$ инволюция является стандартной антиподальной, так что факторпространство является вешественньм проективным пространством.

(б) Сушествует подкомпакт $Y \subset \mathbb{R}^{m}$ такой, что $X$ и $Y$ квазигомеоморфны, т.е. $\forall \varepsilon>0$ сушествуют $\varepsilon$-отображения $X \rightarrow Y$ и $Y \rightarrow X$.

(в) Компакт $X$ вложим в $\mathbb{R}^{m}$.

Очевидно, что $($ в) $\Rightarrow(б),($ в) $\Rightarrow(\mathrm{a})$. В работе [38] замечено, что для случая, когда $X$ есть $n$-мерньй полиэдр и $n \leqslant m-3,3 n<2 m-4$, все три условия равносильны. Это замечание является непосредственным следствием теоремы Вебера [87], в которой обобщены результаты Хефлигера [34] с категории гладких многообразий на категорию полиэдров. Кроме того, в работе [38] построен пример компакта, которьй удовлетворяет условию (б), но не удовлетворяет условию (в). В работе [77] построен пример компакта, удовлетворяюшего условию (а) при $m=2$, но не удовлетворяющего условию (в). Таким компактом оказался 3-адический соленоид. Направление для последуюших обобщений дается работой М. А. Штанько [74].

Остановимся на структурепервой части работы. В разделе 1 излагаются необходимые предварительные сведения, которые включают в себя элементы теории погружений, в том числе принщип $C^{0}$-плотности Смейла-Громова, связь теории погружений со стабильньми гомотопическими группами сфер, отображение Кана-Придди, инвариант Хопфа, обобщенный инвариант Хопфа, теорему Р. Коэна о погружении гладких многообразий. Основным инструментом исследования выступает ЕНР-последовательность Джеймса-Уайтхеда. В разделах 2 и 3 изложены доказательства предложений 1 и 2 , и в заключительном разделе 4 напоминаются необходимые сведения из геометрической топологии, что приводит к доказательствам теорем 1 и 2 . Здесь используется спектральньй критерий вложимости компактов в евклидовы пространства, необходимая модификация которого (ср. [75], [73]), содержится в [8]. Заметим, что используется лишш более легкая часть критерия, связанная лишш с достаточшыми (необходимые условия опускаются) условиями вложения компактов.

\section{1. Предварительные сведения}

Пусть $\varphi: N^{n} \rightarrow \mathbb{R}^{n+m}$ - гладкое погружение, причем многообразие $N^{n}$ ориентировано. На пространстве погружений $n$-мерных многообразий введем отношение кобордантности. Скажем, что два погружения $\varphi_{0}, \varphi_{1}$ связаны кобордизмом, $\varphi_{0} \sim \varphi_{1}$, если существует погружение $\Phi:\left(W^{n+1}, \partial W=N_{0}^{n} \cup N_{1}^{n}\right) \rightarrow\left(\mathbb{R}^{n+m+1} \times[0,1]\right)$ такое, что выполнены граничные условия $\varphi_{i}=\left.\Phi\right|_{N_{i}^{n}}: N_{i}^{n} \rightarrow \mathbb{R}^{n+m} \times\{i\}, i=0,1$, причем 
выполнены стандартные условия согласования ориентаций на $W$ и $\partial W$. Множество классов кобордизмов погруженных ориентированных многообразий образует абелеву группу относительно операции дизъюнктного объединения погружений. Эту группу обозначим через $\mathrm{Imm}^{s o}(n, m)$. Если условие ориентируемости $N^{n}$ опустить, то определена другая разновидность группы кобордизмов погружений, которую обозначим через $\operatorname{Imm}^{o}(n, m)$. В предположении, что погружение $\varphi$ является оснашеншым, т.е. зафиксирован изоморфизм нормального расслоения $\nu(\varphi)$ и суммы Уитни $m$ экземпляров тривиального одномерного расслоения $\varepsilon$ над $N, \nu=k \varepsilon$, определяются групшы $\operatorname{Imm}^{\text {so; } f r}(n, m)$. При этом требуется, чтобы погружение $\Phi$, определяюшее кобордизм двух погружений $\varphi_{i}, i=0,1$, также было оснащенным, согласованно с оснашением границы. По аналогии с групшами $\operatorname{Imm}^{s o ; f r}(n, m)$ определены групшы $\operatorname{Im}^{o ; f r}(n, m)$. При этом требуется фиксировать изоморфизм $\nu(\varphi)=m \varkappa$, где $\varkappa$ - какое-нибудь одномерное расслоение над $N$. Заметим, что в случае нечетного $m$ расслоение $\varkappa$ необходимо совпадает с ориентируюшим расслоением над $M$. Определение кобордантности погружений дается аналогично, при этом используется равенство $\nu(\Phi)=m \varkappa \mathrm{c}$ соответствующими условиями согласования на $\partial W$. По построению очевидно, что $\operatorname{Imm}^{s o ; f r}(n, 1)=\operatorname{Imm}^{s o}(n, 1), \operatorname{Imm}^{o ; f r}(n, 1)=\operatorname{Imm}^{o}(n, 1)$. В работе [88] найдена модификация конструкции Понтрягина [65] для оснащенных кобордизмов, позволяющая доказать следующие изоморфизмы. (По поводу ориентируемого случая см. также [28].)

ПРЕДЛОЖЕНИЕ 1.1. Справедливы следующие изоморфизмы:

$$
\begin{gathered}
\operatorname{Imm}^{\text {so;fr }}(n, m)=\operatorname{Imm}^{\text {so }}(n, 1)=\lim _{k \rightarrow \infty} \pi_{n+k}\left(S^{k}\right)=\pi_{n+1}\left(\Omega^{\infty} \Sigma^{\infty}\left(S^{1}\right)\right) \\
\operatorname{Imm}^{o}(n, 1)=\lim _{k \rightarrow \infty} \pi_{n+k}\left(\Sigma^{k-1} \mathbb{R P}^{\infty}\right)=\pi_{n+1}\left(\Omega^{\infty} \Sigma^{\infty} \mathbb{R P}^{\infty}\right) .
\end{gathered}
$$

Для краткости введем обозначение $\lim _{k \rightarrow \infty} \pi_{n+k}\left(S^{k}\right)=\Pi_{n}$. Рассмотрим конкретные применения этой теоремы. В случае $n=1$ группа кобордизма $\operatorname{Imm}^{s o}(1,1)$ представлена ориентированной кривой (вообше говоря, несвязной), погруженной в плоскость. Изоморфизм $\operatorname{Im}^{s o}(1,1)=\mathbb{Z} / 2$ сопоставляет погруженной кривой общего положения четность числа ее точек самопересечения. Аналогично, $\operatorname{Im}^{o}(1,1)=\mathbb{Z} / 2$. В случае $n=2$ имеем изоморфизм $\operatorname{Imm}^{s o}(2,1)=\mathbb{Z} / 2$, см. [31], [88], [65], $\operatorname{Im}^{o}(2,1)=$ $\mathbb{Z} / 8$, см. [31], [88], [53]. Естественный гомоморфизм $\operatorname{Imm}^{s o}(2,1) \rightarrow \operatorname{Imm}^{o}(2,1)$ определяется мономорфизмом $\mathbb{Z} / 2 \hookrightarrow \mathbb{Z} / 8$. Образующая групшы $\operatorname{Imm}^{o}(2,1)$ представлена погружением Боя $\psi: \mathbb{R P}^{2} \rightarrow \mathbb{R}^{3}$, см. [27]. Образующая групшы $\operatorname{Imm}^{s o}(2,1)$ представлена некоторым погружением тора. Один из возможных вариантов выбора этого погружения, которое, вдобавок, удовлетворяет дополнительным замечательным свойствам, строится в разделе 5 на рис. 2-4. Это погружение называется погружением Константинова.

Операции композиции $f_{2} \circ f_{1}: S^{n_{1}} \rightarrow S^{n_{2}} \rightarrow S^{n_{3}}, \Pi_{n_{1}-n_{2}} \times \Pi_{n_{2}-n_{3}} \rightarrow \Pi_{n_{1}-n_{3}}$ в кольце стабильных гомотопических групп сфер соответствует операция прямого произведения погружений, задающих соответствующие элементы групп кобордизмов. Тем самым, определена операция

$\operatorname{Imm}^{s o}\left(n_{1}-n_{2}, 1\right) \times \operatorname{Imm}^{s o}\left(n_{2}-n_{3}, 1\right) \rightarrow \operatorname{Imm}^{s o ; f r}\left(n_{1}-n_{3}, 2\right) \approx \operatorname{Imm}^{s o}\left(n_{1}-n_{3}, 1\right)$. 
В частности, для образующей $\eta_{1} \in \operatorname{Imm}^{s o}(1,1)$ произведение $\eta_{1}^{2} \in \operatorname{Imm}^{s o}(2,1)$ также является образуюшей. Построение композиций Тоды на языке теории погружений проведено в работе [46].

Далее нам потребуется гомоморфизм

$$
\delta: \operatorname{Imm}^{s o}(n, 1) \rightarrow \operatorname{Imm}^{o}(n-1,1),
$$

который является обратным расщепляющим отображением для гомоморфизма Кана-Придди [2]. (По поводу связи с прочими конструкциями см. [54], [46].) Для построения гомоморфизма $\delta$ воспользуемся следуюшим утверждением, доказанньм Хиршем в [35].

ПреДЛОЖенИЕ 1.2. Пусть $f: N^{n} \rightarrow \mathbb{R}^{n+k}$ - погружение такое, что нормальное расслоение $\nu(f) \kappa$ погруженному многообразию $f(N)$ изоморфно прямой сумме $\mu \oplus \epsilon$, где $\epsilon$ - тривиальное одномерное расслоение $u k \geqslant 2$. Тогда существует регулярная гомотопия, которая связивает погружение $f$ с погружением $I \circ g, g: N^{n} \rightarrow \mathbb{R}^{n+k-1}, I: \mathbb{R}^{n+k-1} \hookrightarrow \mathbb{R}^{n+k}$, для которого $\nu(g)=\mu$. При этом погружение g удается определить с точностью до кобордантного.

Приступим к определению $\delta$. Пусть $\varphi: N^{n} \rightarrow \mathbb{R}^{n+1}$ - погружение, представляюшее элемент из групшы $\operatorname{Imm}^{s o}(n, 1)$. Согласно теореме о приведении в обшее положение отображение $\varphi$ регулярно гомотопно погружению общего положения $\varphi^{\prime}$, для которого множество $\Delta$ двукратных точек самопересечения представляет собой образ погруженного многообразия $\operatorname{dim}(\Delta)=n-1$. Далее, не ограничивая общности, мы предполагаем, что с самого начала $\varphi=\varphi^{\prime}$. Согласно наблюдению работы [47] (см. также [12]) нормальное расслоение $\nu=\nu(\Delta)$ каноническим образом представлено как сумма двух одномерных расслоений $\varkappa \oplus \epsilon$, где $\epsilon$ - тривиальное одномерное расслоение, $\varkappa$ - такое одномерное расслоение, что $w_{1}(\varkappa)$ совпадает с ориентируюшим классом $\Delta$. По предложению 1.2 существует погружение $g: \Delta \rightarrow \mathbb{R}^{n}$, представляющее требуемьй класс регулярной гомотопии. Отображение $\delta$ определено по формуле $\delta(\varphi)=g$. В случае $\delta: \operatorname{Imm}^{s o}(1,1) \rightarrow \operatorname{Imm}^{o}(0,1)$ имеем $\operatorname{Imm}^{s o}(1,1)=\mathbb{Z} / 2, \operatorname{Imm}^{o}(0,1)=\mathbb{Z} / 2$ и отображение $\delta$ ставит в соответствие погруженной кривой $\varphi: N^{1} \rightarrow \mathbb{R}^{2}$ класс кобордизма погружения $g: \Delta \rightarrow \mathbb{R}^{1}$ неориентированного нульмерного многообразия, который определяется четностью числа компонент. В случае $\delta: \operatorname{Imm}^{s o}(2,1) \rightarrow \operatorname{Imm}^{o}(1,1)$ погруженной поверхности $\varphi: N^{2} \rightarrow \mathbb{R}^{3}$ ставится в соответствие погружение $g: \Delta \rightarrow \mathbb{R}^{2}$, которое получено регулярной гомотопией оснашенной кривой двукратных точек $\Delta$ погружения $\varphi$ в пространстве $\mathbb{R}^{3}$ к погружению в плоскость, при этом первый вектор оснашения переводится регулярной гомотопией в вектор, перпендикулярный к плоскости, второй вектор переводится в касательный к плоскости. Инвариант кобордизма в группе $\operatorname{Imm}^{o}(1,1)=\mathbb{Z} / 2$ определяется числом точек самопересечения кривой $g(\Delta)$ в плоскости $\mathbb{R}^{2}$.

Напомним конструкцию стабильного инварианта Хопфа $h: \operatorname{Imm}^{s o}(n, 1) \rightarrow \mathbb{Z} / 2$. Исходное определение можно найти, например, в [59], предложенную ниже модификацию в рамках теории погружений можно найти в работах [12], [24], [46], [50], [80]. Пусть $[\varphi] \in \operatorname{Imm}^{s o}(n, 1) ; \psi=\delta(\varphi), \psi \in \operatorname{Imm}^{o}(n-1,1), \psi: M^{n-1} \rightarrow \mathbb{R}^{n}, M^{n-1}=$ $\Delta(\varphi)$. Определим характеристическое число $\left\langle w_{1}^{n-1}(M) ;[M]\right\rangle$ и обозначим это число через $h(\varphi)$. 
Лемма 1.3. Отображсене $h: \operatorname{Imm}^{s o}(n, 1) \rightarrow \mathbb{Z} / 2$ корректно определено.

ДокАЗАТЕльство. Пусть $\varphi_{1}: N_{1} \rightarrow \mathbb{R}^{n+1}, \varphi_{2}: N_{2} \rightarrow \mathbb{R}^{n+1}$ - два различных представителя элемента $[\varphi] \in \operatorname{Im}^{s o}(n, 1)$. Рассмотрим погружения $\psi_{1}, \psi_{2}$, построенные по соответствуюшим многообразиям кратных точек. Заметим, что многообразия $\Delta\left(\varphi_{1}\right)$ и $\Delta\left(\varphi_{2}\right)$ кобордантны, поскольку $\varphi_{1}$ и $\varphi_{2}$ по условию связаны кобордизмом. Соответствуюшие характеристические числа кобордантных многообразий совпадают. Лемма доказана.

ОПРЕДЕЛЕНИЕ 1.4. Скажем, что многообразие $M^{n-1}$ допускает ретракиию порядка $d$ (относительно коцикла $\varkappa$ ), если сушествует отображение $\bar{r}: M^{n-1} \rightarrow$ $\mathbb{R} \mathrm{P}^{n-d-2}$ такое, что коммутативна диаграмма

$$
\begin{gathered}
M^{n-1} \stackrel{r}{\longrightarrow} \mathbb{R P}^{\infty} \\
\bar{r} \nearrow_{I} I, \\
\mathbb{R P}^{n-d-2}
\end{gathered}
$$

в которой $I$ - стандартное вложение проективного подпространства, а отображение $r$ определяется коциклом $\varkappa \in H^{1}(M ; \mathbb{Z} / 2)$.

По определению произвольное многообразие $M$ допускает ретракцию порядка -1 . Действительно, по теореме о клеточной аппроксимации произвольное отображение $r: M^{n-1} \rightarrow \mathbb{R P}^{\infty}$ гомотопно отображению $\bar{r}: M \rightarrow \mathbb{R P}^{n-1}$ в остов проективного пространства размерности $n-1$.

Определим понятие обобшенного инварианта Хопфа и опишем ЕНР-последовательность, связанную с этим инвариантом, следуя построениям работы [47]. Вначале определим следуюшие разновидности групп кобордизма погружений. Опишем лишь представителей соответствующих груп, опуская описания отношения кобордантности. Группу кобордизма оснашенных вложений $\varphi: M^{n} \rightarrow \mathbb{R}^{m+n} n$-многообразий в коразмерности $m$ обозначим $E m b^{s o ; f r}(n, m)$. В соответствии с конструкцией Понтрягина имеется изоморфизм $E m b^{s o ; f r}(n, m)=\pi_{m+n}\left(S^{m}\right)$. Обозначим через $\operatorname{Imm}^{s o ; f r}(n, m ; r)$ группу кобордизма оснащенных вложений $\psi: M^{n} \subset \mathbb{R}^{m+n+r}$ таких, что отображение $\varphi=p \circ \psi: M^{n} \rightarrow \mathbb{R}^{m+n}$ является погружением, где $p: \mathbb{R}^{m+n+r} \rightarrow \mathbb{R}^{m+n}$ - стандартная проекция. При $0<m<2 n-2$ и произвольном $r \geqslant 0$ имеем изоморфизм $\operatorname{Imm}^{s o ; f r}(n, m ; r)=\pi_{m+n+r}\left(S^{r+m}\right)$. По поводу доказательств здесь и далее в этой конструкции см. [47].

Обозначим через $\operatorname{Im} m^{o ; f r}\left(n, m ; \mathbb{R} \mathrm{P}^{r}\right)$ группу кобордизма неориентированных оснащенных погружений $\varphi: M^{n} \rightarrow \mathbb{R}^{n+m}$, для которых зафиксирован изоморфизм $\nu(\varphi)=$ $m \varkappa$ (неориентированное оснашение) и таких, что многообразие $M^{n}$ допускает ретракцию порядка $n-r-1$ по отношению к коциклу, классифицируюшему расслоение $\varkappa$. При определении отношения кобордантности также предполагается, что многообразие с краем, задаюшее кобордизм, допускает ретракцию того же порядка, согласованную с ретракциями границы. Имеем очевидные равенства $\operatorname{Imm}^{s o ; f r}(n, m ; \infty)=$ $\operatorname{Imm}^{s o ; f r}(n, m), \operatorname{Imm}^{o ; f r}\left(n, m ; \mathbb{R P}{ }^{\infty}\right)=\operatorname{Imm}^{o ; f r}(n ; m)$.

Построенные группы связаны между собой длинной точной последовательностью:

(2) $\operatorname{Imm}^{o ; f r}\left(n-m-1, m+1 ; \mathbb{R} \mathrm{P}^{r}\right) \stackrel{P^{r+1}}{\longrightarrow} \operatorname{Emb}^{\text {so;fr }}(n, m) \stackrel{E^{r}}{\longrightarrow} \operatorname{Imm}^{s o ; f r}(n, m ; r)$

$$
\stackrel{H^{r}}{\longrightarrow} \operatorname{Im} m^{o ; f r}\left(n-m, m ; \mathbb{R P}^{r-1}\right) \stackrel{P^{r}}{\longrightarrow} \operatorname{Emb}^{\text {so } ; f r}(n-1 ; m-1) \rightarrow \cdots,
$$


в которой связуюший гомоморфизм $P$ определен лишь в предположении $0<m \leqslant$ $2 n-2$.

Опишем гомоморфизмы, входяшие в последовательность (2). Гомоморфизм $E^{r}$ индуцирован естественным включением $\mathbb{R}^{m+n} \subset \mathbb{R}^{m+n+r}$. Гомоморфизм $H^{r}$ сопоставляет классу кобордизма оснашенного погруженного многообразия $\varphi: N^{n} \rightarrow \mathbb{R}^{n+m}$ класс кобордизма погруженного многообразия $\Delta$ двукратных точек, причем фиксируется каноническое расшепление нормального расслоения (в евклидовом пространстве $\left.\mathbb{R}^{n+m}\right)$ к $\Delta$ в сумму одномерных. Подробнее, определен канонический изоморфизм нормального расслоения $\nu(\Delta)$ и расслоения $m \varepsilon \in \oplus m \varkappa$, где $\varkappa$ есть 1-расслоение, ассоциированное с коциклом $w_{1}(\varkappa)$ канонического накрытия $\bar{\Delta} \rightarrow \Delta$. Согласно предложению 1.2 определено погружение (с точностью до кобордизма) $\psi: \Delta^{n-m} \rightarrow \mathbb{R}^{n}$ и изоморфизм нормального расслоения $\nu(\psi)=m \varkappa$, тем самым построен элемент из групшы $\operatorname{Imm}^{o ; f r}(n-m ; m)$.

Отображение $f: \Delta^{n-m} \rightarrow \mathbb{R} \mathrm{P}^{\infty}$, классифицируемое коциклом $w_{1}(\varkappa)$, допускает ретракцию порядка $r$, поскольку является многообразием двойных точек самопересечения погружения, которое спроектировано из вложения из коразмерности $r$. Это показьвает, что погружение $\psi$ определяет элемент из групшы $\operatorname{Im}^{o} ; f r\left(n-m, m ; \mathbb{R P}^{r-1}\right)$.

В частном случае $m=1, r=\infty$ гомоморфизм

$$
\operatorname{Imm}^{s o}(n, 1)=\operatorname{Imm}^{s o ; f r}(n, 1 ; r) \stackrel{H^{\infty}}{\longrightarrow} \operatorname{Imm}^{o ; f r}\left(n-1,1 ; \mathbb{R P}^{\infty}\right)=\operatorname{Im}^{o}(n-1,1)
$$

обратен к гомоморфизму Кана-Придли [3]. В общем случае гомоморфизм $H^{r}$ называется обобщенным гомоморфизмом Хопфа. В случае $n=m, r=\infty$ композиция

$$
\operatorname{Im}^{s o}(n, 1) \rightarrow \operatorname{Imm}^{s o ; f r}(n, m) \rightarrow \operatorname{Im}^{o ; f r}(0, n)=\mathbb{Z} / 2
$$

совпадает со стабильным гомоморфизмом Хопфа $h$, определенным ранее. Конструкцию гомоморфизма $P$ мы опустим, так как для наших построений она непосредственно не понадобится. Она оказьвается полезной для алгебраических выгислений, например, может быть использована при доказательстве утверждения, сформулированного ниже в примере 1.6. Необходимые приложения точной последовательности основаны на следующем предложении.

ПРЕДЛОЖЕНИЕ 1.5. Пусть $\varphi: N^{n} \rightarrow \mathbb{R}^{n+1}$ - погружсение общего положения. Пусть $d<\left[\frac{n}{2}\right]$. Пусть каждая компонента связности погруженного многообразия $\delta(\varphi)=\psi: \Delta^{n-1} \rightarrow \mathbb{R}^{n}$ допускает ретракиию порядка $d$. Тогда композииия $I \circ \varphi: N^{n} \rightarrow \mathbb{R}^{n+1} \subset \mathbb{R}^{2 n-d}$ аппроксимируется $C^{\infty}$-близким вложением $\bar{\varphi}: N^{n} \subset \mathbb{R}^{2 n-d},\|\bar{\varphi}-I \circ \varphi\|<\varepsilon$.

ДокАЗАтЕльство. Погружение $\varphi$ определяет элемент в групе $\operatorname{Imm}^{s o}(n, 1)$. Рассмотрим включение $J: \mathbb{R}^{n+1} \subset \mathbb{R}^{n+\left[\frac{n}{2}\right]+1}$ и индуцированное отображение $J_{\left[\frac{n}{2}\right]+1}: \operatorname{Imm}^{s o}(n, 1) \rightarrow \operatorname{Imm}^{s o ; f r}\left(n,\left[\frac{n}{2}\right]+1 ; \infty\right)$. Приведем погружение $J \circ \varphi$ в общее положение в пространстве $\mathbb{R}^{n+\left[\frac{n}{2}\right]+1}$. Тогда многообразие $\Delta^{n-\left[\frac{n}{2}\right]-1}$ этого погружения становится вложенным и само допускает ретракцию порядка $d$, относительно коцикла, классифицирующего каноническое 2 -накрытие $\bar{\Delta}^{n-\left[\frac{n}{2}\right]} \rightarrow \Delta^{n-\left[\frac{n}{2}\right]}$. Следовательно, $\operatorname{Im}\left(J_{\left[\frac{n}{2}\right]+1}\right)$ лежит в подгруппе $\operatorname{Imm}^{s o ; f r}\left(n,\left[\frac{n}{2}\right]+1 ; n-\left[\frac{n}{2}\right]-d-1\right)$. 
Отображение $J^{\prime} \circ J \circ \varphi: N^{n} \rightarrow \mathbb{R}^{2 n-d}, J^{\prime}: \mathbb{R}^{n+\left[\frac{n}{2}\right]+1} \subset \mathbb{R}^{2 n-d}$ становится вложением после соответствующей малой деформации. Предложение 1.5 доказано.

Пользуясь предложением 1.5, сформулируем рецепт решения задачи дискретной реализации погружения $\varphi: N^{n} \rightarrow \mathbb{R}^{n+1}$ в пространстве $\mathbb{R}^{2 n-d}$. Вначале по классу кобордизма погруженного многообразия двукратных точек вычисляем обобщенньй инвариант Хопфа, принимающий значения в группе $\operatorname{Im}^{o ; f r}(d, n-d)$, как частичное алгебраическое препятствие в задаче устранения многообразия двукратных точек погружения $N^{n} \rightarrow \mathbb{R}^{n+1} \rightarrow \mathbb{R}^{2 n-d}$. Удобно воспользоваться следующей коммутативной диаграммой.

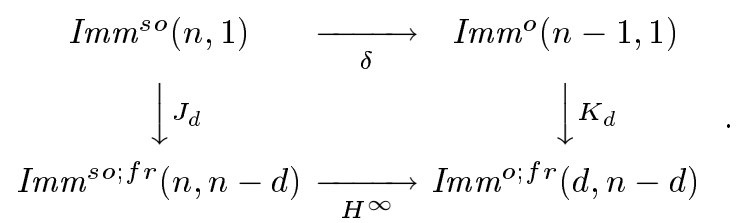

Пусть погружение $\varphi$, представляющее элемент $[\varphi] \in \operatorname{Imm}^{s o}(n, 1)$, таково, что $H^{\infty} \circ J_{d}([\varphi])=0$. Рассмотрим погружение $\delta(\varphi)=\psi: M^{n-1} \rightarrow \mathbb{R}^{n}$. Задача дискретной реализации погружения $\varphi$ в пространстве $\mathbb{R}^{2 n-d}$ имеет положительное решение, если погружение $\psi$ допускает ретракцию порядка $d$.

ПримеР 1.6. Согласно вычислениям [82] гомоморфизм надстройки

$$
E^{3}: \pi_{13+15}\left(S^{13}\right) \rightarrow \Pi_{15}
$$

является эпиморфизмом. Справедливо равенство $\Pi_{15}=\mathbb{Z} / 3 \oplus \mathbb{Z} / 5 \oplus \mathbb{Z} / 16$. Пусть $\alpha \in \Pi_{15}$ - образуюшая 2-компоненты.

Используя точность ЕНР-последовательности, получаем равенство $0=\delta \circ I_{2}(\alpha) \in$ $\operatorname{Imm}^{o ; f r}(2,13)$. В частности, имеем $h(\alpha)=0$. Следовательно, погружение $\varphi: N^{15} \rightarrow$ $\mathbb{R}^{16}$, представляюшее элемент $\alpha$, допускает ретракцию порядка 0 и композиция $N^{15} \rightarrow \mathbb{R}^{16} \rightarrow \mathbb{R}^{30}$ апшроксимируется вложением.

Нам потребуется одна из основных и наиболее сложных теорем теории погружений, доказанная Р. Коэном в [20].

ПРЕДЛОЖЕНИЕ 1.7. Обозначим через $\alpha(m)$ число единии в двоичной записи числа $m$. Произвольное замкнутое многообразие $M, \operatorname{dim}(M)=m$, допускает погружение $f: M^{m} \rightarrow \mathbb{R}^{2 m-\alpha(m)}$.

ЗАмЕЧАниЕ. Пользуясь предложением 1.2 , легко дать другую эквивалентную формулировку предложения 1.7 . Пусть $\nu \rightarrow M^{m}$-нормальное расслоение стабильной размерности, $\operatorname{dim}(\nu)=m+1$. Тогда расслоение $\nu$ допускает, по меньшей мере, $\alpha(m)+1$ линейно независимых сечений.

Следуюшее утверждение известно как принцип $C^{0}$-плотности и было подмечено М. Громовым в [30; предложение 1.2.2].

ПРЕДЛОЖЕНИЕ 1.8. Пусть $f: M \rightarrow(N ; b)$ гладкое погружение замкнутьх многообразий, причем многообразие $N$ снабжено метрикой $b, \operatorname{dim}(M)<\operatorname{dim}(N)$. Пусть $g: M \rightarrow N$ есть $C^{0}$-отображение, гомотопное отображению $f$. Тогда для любого $\varepsilon>0$ найдется погружсение $h: M \rightarrow N$, для которого $\|h-g\|_{C^{0}}<\varepsilon$ в метрике пространства отображений, индуцированной метрикой $b$. 


\section{2. Дискретная реализация отображений $n$-мерной сферы $S^{n} \rightarrow S^{n}$ в евклидовом пространстве $\mathbb{R}^{2 n}$}

Пусть $I: S^{n} \rightarrow \mathbb{R}^{n+1}, J: \mathbb{R}^{n+1} \rightarrow \mathbb{R}^{2 n}, H=J \circ I: S^{n} \rightarrow \mathbb{R}^{2 n}$ - стандартные вложения. Для доказательства предложения 1.А нам потребуется следующее определение. В большей обшности это определение сформулировано в [8].

ОПРЕДЕЛЕНИЕ 2.1. Пусть $f: S^{n} \rightarrow S^{n}$ есть $C^{0}$-отображение. Скажем, что $f$ 几окально дискретно реализуется $(\mathrm{rel} I)$ или, иначе, $C^{0}$-аппроксимируется погружением, если для любого $\varepsilon>0$ сушествует погружение $g=g(\varepsilon): S^{n} \rightarrow \mathbb{R}^{n+1}$ такое, что

$$
\sup _{a \in S^{n}}\|I \circ f(a)-g(a)\|<\varepsilon .
$$

Скажем, что отображение $f$ локально изотопически реализуется в $\mathbb{R}^{n+1}$ ( rel $I$ ) или, иначе, $C^{0}$-аппроксимируется в $\mathbb{R}^{n+1}$ семейством погружений, если сушествует регулярная гомотопия (т.е. гомотопия погружений)

$$
\left.\left.\Phi_{t}: S^{n} \rightarrow \mathbb{R}^{n+1}, \quad t \in\right] 0 ; 1\right]
$$

такая, что $\Phi_{t}$ продолжается по непрерьвности при $t \rightarrow+0$ до непрерывного отображения $\Phi_{0}: S^{n} \rightarrow \mathbb{R}^{n+1}$, причем $\Phi_{0}=I \circ f$.

ЛЕмма 2.2. Произвольное отображсние $f: S^{n} \rightarrow S^{n}$ локально изотопически реализуется $(\operatorname{rel} I)$ в пространстве $\mathbb{R}^{n+1}$.

Доказательство изложено в [8].

Приступим к доказательству первого главного результата (формулировку см. во введении).

ДОКАЗАТЕЛЬСТВО ПРЕДЛОЖЕНИЯ 1 .А. По лемме 2.2 сушествует погружение общего положения $g: S^{n} \rightarrow \mathbb{R}^{n+1}$, сколь угодно близкое к композиции $I \circ f: S^{n} \rightarrow \mathbb{R}^{n+1}$ в $C^{0}$-топологии. Рассмотрим погружение $\psi=\delta(g): \Delta^{n-1} \rightarrow \mathbb{R}^{n}$ (см. предложение 1.2) и разбиение многообразия $\Delta=\bigcup_{i} \Delta(i)$ на компоненты связности. Для погружения каждой связной компоненты рассмотрим инвариант Хопфа $h(i)=$ $\left\langle w_{1}^{n-1}(\Delta(i)) ;[\Delta(i)]\right\rangle \in \mathbb{Z} / 2$, см. лемму 1.3. В работах [24], [46], [50] замечено, что для каждого $n \neq 1,3,7$ вьполнено равенство $h(i)=0$. Доказательство этого использует результат Адамса [2] об инвариантах Хопфа.

ЛЕмма 2.3. При условиях $n \neq 1,3,7$ для кажсдого $i$ многообразие $\Delta(i)$ допускает ретракцию порядка 0 относительно коцикла, классифицирующего каноническое накрытие $\bar{\Delta}(i) \rightarrow \Delta(i)$, в смысле определения 1.4 .

ДокАЗАТЕльство. Построим диаграмму отображений, задающую ретракцию, в которой $r$ задан ориентирующим 1 -коциклом, $\bar{r}$ - ретракция порядка 0 :

$$
\begin{gathered}
\Delta^{n-1}(i) \stackrel{r}{\longrightarrow} \mathbb{R} \mathrm{P}^{\infty} \\
\bar{r} \searrow \\
\mathbb{R} \mathrm{P}^{n-2}
\end{gathered}
$$


По теореме о клеточной аптроксимации можно построить гомотопию $F$, переводяшую отображение $r$ в некоторое $r_{1}$, такую, что $\operatorname{Im}\left(r_{1}\right) \subset \mathbb{R P}^{n-1} \subset \mathbb{R} \mathrm{P}^{\infty}$. Перейдем к $\mathbb{Z} / 2$-эквивариантному отображению двулистных накрытий $r_{1}^{!}: \widehat{\Delta}^{n-1}(i) \rightarrow S^{n-1}$. Воспользуемся тем, что $r$ - ориентирующий коцикл. Многообразие $\widehat{\Delta}^{n-1}(i)$ ориентировано, и определена целочисленная степень отображения $\operatorname{deg}\left(r_{1}^{!}\right)$, причем $\operatorname{deg}\left(r_{1}^{!}\right)=$ $\operatorname{deg}\left(r_{1}\right)(\bmod 2)$. По теореме Адамса $\operatorname{deg}\left(r_{1}\right)=0(\bmod 2)$. Рассмотрим два случая. При $n=0(\bmod 2)$ многообразие $\mathbb{R} \mathrm{P}^{n-1}$ ориентировано, степень отображения $r_{1}^{!}$не зависит от выбора гомотопии $F$ и равна 0.

В случае $n=1(\bmod 2)$ условие $\operatorname{deg}\left(r_{1}\right)=0(\bmod 2)$ позволяет определить эквивариантную гомотопию $G$, переводящую $r_{1}^{!}$в $r_{2}^{!}$, причем $\operatorname{Im}\left(r_{2}^{!}\right) \subset S^{n-2} \subset S^{n-1}$. Переходя к факторотображению $\bar{r}=r_{2}^{!} / T$, где через $T$ обозначена инволюция на двулистном накрытии, получаем доказательство леммы 2.3 .

По лемме 2.3 , предложению 1.5 и конструкции, изложенной в разделе 1 сразу после предложения 1.5 , отображение $f \circ H: S^{n} \rightarrow \mathbb{R}^{n+1} \subset \mathbb{R}^{2 n}$ может быть сколь угодно близко $C^{0}$-аптроксимировано гладким вложением. Предложение 1.А доказано.

ДоКАЗАТЕЛЬСТво ПРЕДЛОЖЕНИя 1.Б. Построим отображения $S^{1} \rightarrow S^{1}, S^{3} \rightarrow$ $S^{3}, S^{7} \rightarrow S^{7}$, не реализуюшиеся в пространствах $\mathbb{R}^{2}, \mathbb{R}^{6}, \mathbb{R}^{14}$ соответственно. Результат для $n=1$ элементарен, в [75] замечено, что двулистное накрытие $S^{1} \rightarrow S^{1}$ не реализуется в плоскости. Изложим построение отображения $\Xi: S^{7} \rightarrow S^{7}$ с указанными свойствами. Построение для $S^{3}$ совершенно аналогично.

Конструкция. Пусть $\varphi: \mathbb{R P}^{6} \rightarrow S^{7}$ - погружение общего положения. Согласно теории погружений такое погружение сушествует, поскольку определена тривиализация расслоения $\tau \oplus \varkappa \rightarrow \mathbb{R P}^{6}$, где $\tau \oplus \varkappa-$ сумма Уитни касательного расслоения к $\mathbb{R} \mathrm{P}^{6}$ и одномерного ориентируюшего расслоения. Выбор такой тривиализации определяет погружение $\varphi$ с точностью до регулярной гомотопии. Далее, определим погружение $\widehat{\varphi}: S^{6} \rightarrow S^{7}$ как $\widehat{\varphi}=\varphi$ о $p$, где $p: S^{6} \rightarrow \mathbb{R P}^{6}$ - каноническое 2-накрытие.

Построим гомотопию $\xi_{t}: S^{6} \times I \rightarrow S^{7}, I=[0 ; 1]$, такую, что при $t \neq 1 \xi_{t}$ является погружением, $\xi_{0}=\widehat{\varphi}, \xi_{1}$ - отображение в точку и $\xi_{t}$ имеет стандартньй вид в окрестности значения параметра $t=1$. Для этого построения требуется следуюшая лемма.

ЛЕмма 2.4. Любъе два погружения $\xi_{i}: S^{6} \rightarrow \mathbb{R}^{7}, i=1,2$, связаны регулярной гомотопией.

ДокАЗАТЕЛЬСТво. Согласно результатам [78] достаточно доказать равенство $\pi_{6}(S O(7))=0$. Рассмотрим каноническое расслоение $j: S O(8) \rightarrow S^{7}$ со слоем $S O(7)$. Существует сечение $j^{-1}: S^{7} \rightarrow S O(8)$. Для построения $j^{-1}$ можно отождествить $S^{7}$ с унимодулярными числами Кэли $C a^{1}$. По произвольному числу из $C a^{1}$ каноническим образом строится оператор из $S O(8)$, см., например, [66]. Рассмотрим точную последовательность гомотопических групा, индуцированную расслоением $j$ :

$$
\cdots \longrightarrow \pi_{7}(S O(8)) \sharp \stackrel{j_{\sharp}}{\longrightarrow} \pi_{7}\left(S^{7}\right) \stackrel{\partial_{\sharp}}{\longrightarrow} \pi_{6}(S O(7)) \stackrel{i_{\sharp}}{\longrightarrow} \pi_{6}(S O(8)) \longrightarrow \cdots .
$$

В этой последовательности $\operatorname{Im}\left(\partial_{\sharp}\right)=0$, поскольку $j_{\sharp}-$ эпиморфизм. В соответствии с теоремой Ботта имеем $\pi_{6}(S O(8))=0$, см., например, [23]. Равенство $\pi_{6}(S O(7))=0$ и лемма 2.4 доказаны. 
Рассмотрим, пользуясь доказанной леммой, регулярную гомотопию $\xi_{t}$, связываюшую погружение $\widehat{\varphi}$ со стандартным вложением, параметризуюшим малую границу окрестности точки. Гомотопия $\xi_{t}$ задает продолжение отображения $\varphi: \mathbb{R} \mathrm{P}^{6} \rightarrow S^{7}$ на клетку старшей размерности в многообразии $\mathbb{R} \mathrm{P}^{7}$. Тем самым, определено отображение $\Lambda: \mathbb{R P}^{7} \rightarrow S^{7}$. Гомотопию $\xi_{t}$ выберем такой, чтобы отображение $\Lambda$ имело степень 1. Этот выбор осушествляется в соответствии со следуюшими простыми рассуждениями.

Сначала выберем гомотопию $\xi_{t}$ произвольной, что определяет некоторое предварительное отображение $\Lambda_{0}$, для которого $\operatorname{deg}\left(\Lambda_{0}\right)$, вообще говоря, не равно 1 . Далее определим модификации $\Lambda_{1}^{+}$и $\Lambda_{1}^{-}$отображения $\Lambda_{0}$, которые совпадают с $\Lambda_{0}$ всюду, за исключением малой окрестности точки $x_{0} \in \mathbb{R P}^{7}$ - центральной точки клетки старшей размерности. При этом выполнены равенства $\operatorname{deg}\left(\Lambda_{1}^{+}\right)=\operatorname{deg}\left(\Lambda_{0}\right)+1$, $\operatorname{deg}\left(\Lambda_{1}^{-}\right)=\operatorname{deg}\left(\Lambda_{0}\right)-1$.

Для построения модификаций удобно условиться о выборе ориентации на $\mathbb{R P}{ }^{7}$ такой, что отображение $\Lambda_{0}$, ограниченное на малую окрестность $U_{0}$ точки $x_{0}$, является диффеоморфизмом степени +1 . В частности, это определяет ориентацию на гранище $\partial U_{0}$ окрестности. Далее рассмотрим диффеоморфизм $I_{+}:\left(S^{7}, y_{0}\right) \rightarrow\left(S^{7}, \Lambda_{0}\left(x_{0}\right)\right)$ степени 1 . Ограничим $I_{+}$на $\partial V_{0}$, где $V_{0}$ - малая окрестность точки $y_{0} \in S^{7}$ такая, что $I_{+}\left(V_{0}\right)$ лежит внутри $\Lambda_{0}\left(U_{0}\right)$. Заметим, что $\partial V_{0}$ снабжена канонической ориентацией. Рассмотрим регулярную гомотопию $F_{t}: S^{6} \rightarrow \operatorname{Im}\left(\Lambda_{0}\left(U_{0}\right)\right)$ такую, что $F_{0}=\left.I_{+}\right|_{\partial V_{0}}$, $F_{1}=\left.\Lambda_{0}\right|_{\partial U_{0}}$. Выбор параметризаций $S^{6} \times\{0\} \cup\{1\}$ начальных и конечных значений гомотопии произведем так, чтобы отображение

$$
\left.\left.I_{+}\right|_{S^{7} \backslash V_{0}} \bigcup_{\partial U_{0}} F_{t} \bigcup_{\partial V_{0}} \Lambda_{0}\right|_{\mathbb{R P}^{7} \backslash U_{0}}=\Lambda_{1}^{+}
$$

являлось бы отображением ориентированного многообразия в соответствии с правилами выбора ориентации на границах окрестностей $V_{0}$ и $U_{0}$. Отображение $\Lambda_{1}^{+}$является искомым, а отображение $\Lambda_{1}^{-}$строится совершенно аналогично.

Теперь для построения искомого отображения $\Lambda$, для которого $\operatorname{deg}(\Lambda)=+1$, можно начать с произвольного отображения $\Lambda_{0}$ и далее применить одну из описанных модификаций необходимое число раз. Дополнительно потребуем выполнение следующего условия $(\mathrm{Y})$.

$(У)$ Гомотопия $\xi_{t}$ при $t \in[0 ;+\delta]$ определяет тривиализацию погруженной трубчатой окрестности $W$ погружения $\varphi\left(\mathbb{R} \mathrm{P}^{6}\right)$.

Завершим конструкцию и определим $\Xi$ как композицию $\Xi=\Lambda \circ p: S^{7} \rightarrow \mathbb{R P}^{7} \rightarrow S^{7}$. (Здесь допущена некоторая вольность в обозначениях, и через $p: S^{7} \rightarrow \mathbb{R P}^{7}$ обозначено каноническое 2-накрытие.) Требуемое отображение построено.

Приступим к доказательству того, что отображение $\Xi$ не реализуется в пространстве $\mathbb{R}^{14}$. Рассмотрим произвольное гладкое отображение $\Lambda^{\prime}: \mathbb{R} P^{7} \rightarrow S^{7}$, задающее малую $C^{0}$-аппроксимацию отображения $\Lambda$. Технически будет удобно помечать области определений аппроксимируюших отображений нижним индексом 1. Выбор константы $C^{0}$-аппроксимации $\varepsilon_{1}$ будет указан позднее. По свойствам обшего положения, которые всегда можно считать выполненными, после, быть может, дополнительной 
деформации отображения $\Lambda^{\prime}$ стратифицированноеподмногообразие критических значений отображения $\Lambda^{\prime}$ находится в общем положении с однопараметрическим семейством погружений $\xi_{t}$, задаюшим отображение $\Lambda$. Кроме того, $\Lambda^{\prime}$ регулярно вдоль семейства погружений, которое определяет многообразие $\varphi\left(\mathbb{R P}^{6}\right)$ и в точке $\Lambda\left(x_{0}\right)$. Для каждого $t \in\left[\varepsilon_{1} ; 1\left[\right.\right.$ определено множество $L_{t}^{\prime} \subset \mathbb{R} \mathrm{P}_{1}^{7}$ по формуле $L_{t}^{\prime}=\Lambda^{\prime-1}\left(\operatorname{Im}\left[\xi_{t}\right]\right)$. Определено также множество $\widehat{L}_{t}^{\prime} \subset S_{1}^{7}$ по формуле $\widehat{L}_{t}^{\prime}=p^{-1}\left(L_{t}\right)$.

По свойствам общего положения множество $L_{t}^{\prime}$ при всех значениях параметра $t$, кроме, быть может, их конечного числа, наделено структурой погруженного в $\mathbb{R} P_{1}^{7}$ многообразия. Такая структура вводится после разрешения особенностей, связанных с точками самопересечения образа погружения $\xi_{t}$, посредством погружения $q_{t}\left(\widehat{q}_{t}\right)$, для всех значений параметра $t$, за исключением, быть может, конечного множества $\Upsilon$ критических значений параметра. В произвольной малой $\delta$-окрестности критического значения $\tau \in \Upsilon$ погруженные подмногообразия $L_{\tau-\delta}^{\prime}$ и $L_{\tau+\delta}^{\prime}$ отличаются на морсовскую перестройку.

Определим $M^{\prime}=\bigcup_{t} L_{t}^{\prime}, t \in\left[\varepsilon_{1} ; 1-\varepsilon\right]$. Теперь заметим, что из условия (У) и свойств обшего положения вытекает, что при малых значениях параметра $\varepsilon_{1}$, много меншших диаметра регулярной окрестности погружения $\varphi\left(\mathbb{R P}^{6}\right)$ и диаметра окрестности $\Lambda\left(U_{0}\right)$, многообразие $M^{\prime}$ допускает вложение $M^{\prime} \subset M$, где $M$ - замкнутое многообразие, определенное теми же формулами, что и $M^{\prime}$, при $\left.t \in\right] 0 ; 1[$, а в окрестности ]0; $\varepsilon_{2}$ [ (где $\varepsilon_{2}$ - некоторое положительное число, не превьшающее $\varepsilon_{1}$ ) допускающее компактификацию подмногообразием $L_{0}^{\prime}$ - центральным подмногообразием нетривиальной трубчатой окрестности, погруженной в $\mathbb{R} \mathrm{P}_{1}^{7}$ и образованной семейством погруженных подмногообразий $L_{t}^{\prime}$.

По построению многообразия $M$ определены отображение $j: M \rightarrow \mathbb{R} \mathrm{P}_{1}^{7}$ и отображение $q^{\prime}: M^{\prime} \rightarrow S_{1}^{7}, q^{\prime}=\bigcup_{t} \Lambda_{t}^{\prime}: \bigcup_{t} L_{t}^{\prime} \rightarrow \operatorname{Im}\left(\xi_{t}\right)\left(\operatorname{Im}\left(\xi_{t}\right)\right.$ - погруженное подмногообразие в сфере $S^{7}$ ). Отображение $q^{\prime}$ продолжается до отображения $q: M \rightarrow \mathbb{R} P^{7}$. Кроме того, определено двулистное накрытие $\widehat{M} \rightarrow M$ и отображения $\widehat{q}: \widehat{M} \rightarrow S^{7}$, $\widehat{j}: \widehat{M} \rightarrow S_{1}^{7}$, двулистно накрываюшие $q$ и $j$ соответственно. Построенные выше отображения включены в следуюшую коммутативную диаграмму:

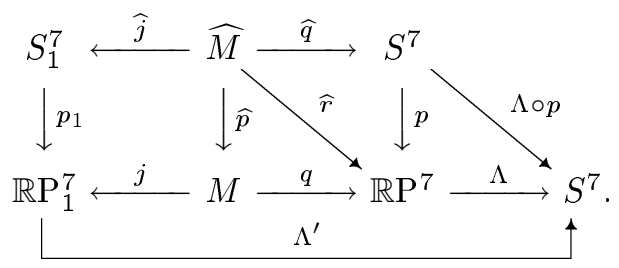

В этой диаграмме $\widehat{r}=q \circ \widehat{p}$. Важно заметить, что $\operatorname{deg}(\widehat{q})=1$.

Теперь рассмотрим предыдушее построение многообразия $\widehat{M}$ в более обшем случае. Предположим, что нам задано некоторое отображение $\Lambda^{\prime \prime}: S_{1}^{7} \rightarrow S^{7}$, аппроксимируюшее композицию $\Lambda \circ p$. Если вьполнено точное равенство $\Lambda^{\prime \prime}=\Lambda \circ p$, то применим описанную выше конструкцию и построим $\widehat{M}$ как двулистное накрытие над $M$. В обшем случае многообразие $M$ не определено, но, повторив аналогичные рассуждения, можно построить многообразие $\widehat{M}$ и определить коммутативную диаграмму гладких отображений 


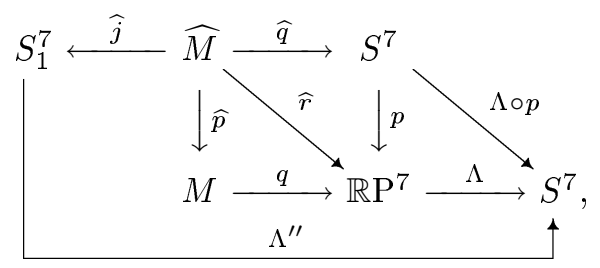

в которой снова $\widehat{r}=q \circ \widehat{p}$ и $\widehat{p}$ - отображения степени 1 .

Вернемся к доказательству предложения 1.Б. Предположим, что для любого $\varepsilon>0$, сушествует отображение $\bar{\wp}$, определяюшее $\varepsilon$-реализацию отображения $\Xi(\mathrm{rel} H)$. Пусть $\pi: U \rightarrow S^{7}$-проекция малой регулярной окрестности на стандартно вложенную сферу $H\left(S^{7}\right)$. Рассмотрим отображение $\wp=\pi \circ \bar{\wp}: S_{1}^{7} \rightarrow S^{7}$. Можно положить $\Lambda^{\prime \prime}=\wp$ и, применив предыдущие построения, получить многообразие $\widehat{M}$, поскольку, не ограничивая обшности, с самого начала можно считать, что вложение § таково, что для отображения $\wp$ выполнены все необходимые условия близости и регулярности. Сформулируем это наблюдение в виде отдельной леммы, доказательство которой очевидно вытекает из построения диаграммы (7).

Лемма 2.5. Существует число $\varepsilon>0$ такое, что по любой $\varepsilon$-реализачии § общего положения отображения $I \circ \Xi: S^{7} \rightarrow \mathbb{R}^{14}$ можно определить коммутативную диаграмму гладких отображсений:

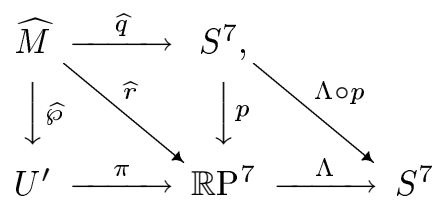

в которой $U^{\prime}=\Lambda^{*}(U)$ - трубчатая окрестность многообразия $\mathbb{R P}^{7}, U^{\prime} \approx$ $\mathbb{R P}^{7} \times D^{7}$, индуцированная как обратный образ окрестности $U$ при отображении $\Lambda, \widehat{\wp}$-отображсение, индуцированное как обратный образ при отображении $\Lambda$ из отображения §० $\widehat{j}: \widehat{M} \rightarrow U^{\prime}$.

Завершим доказательство предложения 1.Б. Предположим, что сушествует дискретная реализация §. Рассмотрим диаграмму (8), которая определена по лемме 2.5 . В работе [26] доказано, что для произвольного $\widehat{M}$, включенного в диаграмму (8) при условии $\operatorname{deg}(\bar{q})=1$, найдется такая пара точек $x, y \in \widehat{M}$, для которой $\widehat{q}(x)=T \circ \widehat{q}(y)$, $\widehat{\wp}(x)=\widehat{\wp}(y)$, где $T: S^{7} \rightarrow S^{7}$ - стандартная инволюция. С другой стороны, вьполнено неравенство $\bar{\wp}(\widehat{j}(x)) \neq \bar{\wp}(\widehat{j}(y))$, поскольку $\widehat{j}(x) \neq \widehat{j}(y)$ на $S_{1}^{7}$, а $\bar{\wp}$ - вложение. Следовательно, и $\widehat{\wp}(x) \neq \widehat{\wp}(y)$, что приводит к противоречию. Предложение 1.Б доказано.

ЗАмЕчАниЕ. Другое доказательство предложения 1.Б (в более слабой формулировке) имеется в работе [69].

\section{3. Дискретная реализация отображения $n$-мерной сферы $S^{n} \rightarrow S^{n}$ в евклидовом пространстве $\mathbb{R}^{2 n-d}$}

В этом разделе будет доказано второе основное утверждение - предложение 2 , сфор- 
мулированное во введении. Предположим, что число $d>0$ задано, и укажем такое число $n$, что произвольное отображение $S^{n} \rightarrow S^{n}$ дискретно реализуется в пространстве $\mathbb{R}^{2 n-d}$. Нам потребуется следующая лемма.

Лемма 3.1. Пусть $g: M^{2^{l+1}-2 d-1} \rightarrow \mathbb{R}^{2^{l+1}-2 d}, d>0,-$ произвольное погружение, представляюшее әлемент $[\varphi] \in \operatorname{Imm}^{o}\left(2^{l+1}-2 d-1,1\right)$. Рассмотрим ориентирующий коцикл $w_{1}(M) \in H^{1}(M ; \mathbb{Z} / 2)$. Тогда при $l \geqslant 3 d$ многообразие $M$ допускает ретракцию порядка $d$ относительно ориентирующего коцикла в смицсле определения 1.4 .

Пользуясь леммой 3.1 , докажем, что при любом $d>0$ произвольное отображение $f: S^{n} \rightarrow S^{n}, n=2^{l+1}-2 d, l>3 d$, дискретно реализуется в пространстве $\mathbb{R}^{2 n-d}$. Пусть $\varepsilon>0$ - произвольно малое положительное число. Мы докажем, что отображение $J \circ f: S^{n} \rightarrow \mathbb{R}^{2 n-d}, J: S^{n} \rightarrow \mathbb{R}^{2 n-d}$, аппроксимируется вложением с константой аппроксимации $\varepsilon$. Рассмотрим композицию $I \circ f: S^{n} \rightarrow \mathbb{R}^{n+1}$, $I: S^{n} \subset \mathbb{R}^{n+1}$. Воспользуемся леммой 2.2 и рассмотрим погружение $h: S^{n} \rightarrow \mathbb{R}^{n+1}$ такое, что $\|h-I \circ f\|_{C^{0}}<\varepsilon / 4$. Рассмотрим погружение $g=\delta(h): M^{n-1} \rightarrow \mathbb{R}^{n}$, построенное в разделе 1 . Согласно лемме 3.1 погружение $g$ допускает ретракцию порядка $d$. Следовательно, согласно предложению 1.5 композиция $J \circ h: S^{n} \rightarrow \mathbb{R}^{2 n-d}$, $J: \mathbb{R}^{n+1} \subset \mathbb{R}^{2 n-d}$, апшроксимируется вложением $\psi: S^{n} \subset \mathbb{R}^{2 n-d},\|J \circ h-\psi\|<\varepsilon / 2$. Утверждение о дискретной реализации и предложение 2 доказаны.

ДОПОЛНЕНИЕ К ДОКАЗАТЕЛЬСТВУ ПРЕДЛОЖЕНИЯ 2. Покажем, используя теорему об изотопической реализации в коразмерности 1, формулировка которой была приведена во введении, что предложение 2 остается справедливо при замене понятия дискретной реализации на понятие изотопической реализации. Для произвольного $d>0$ выберем число $n(d)$ по формуле $n=2^{(3 d+1)+1}-2(d+1)+1$. Согласно доказанному утверждению произвольное отображение $f: S^{n} \rightarrow S^{n}$ дискретно реализуется в пространстве $\mathbb{R}^{2 n-d-1}$. По теореме об изотопической реализации в коразмерности 1 (доказательство которой см. в [11]) отображение $f$ изотопически реализуется в пространстве $\mathbb{R}^{2 n-d}$.

ДокАЗАТЕЛЬСТво ЛЕмМЫ 3.1. Изложим основную идею доказательства, которое можно найти в работе [10]. Пусть $M_{0}^{2^{l}-1} \subset M^{2^{l+1}-2 d-1}$ - подмногообразие, определенное как эйлеров класс (подмногообразие нулей сечения обшего положения) расслоения $\left(2^{l}-2 d\right) \varkappa$. (Эйлеров класс векторного расслоения определяется как множество нулей сечения общего положения. Тем самым, применив теорему Тома о трансверсальности, эйлеров класс можно считать подмногообразием, которое определено с точностью до кобордизма вложенных подмногообразий.) По теореме Коэна (предложение 1.7) стабилизация нормального расслоения $\nu\left(M_{0}\right)$ размерности $2^{l}$ допускает $\alpha\left(2^{l}-1\right)+1$ линейно независимых сечений. Это число при достаточно больших $l$ (например, при $l>3 d$ ) превьшает $2 d$. С другой стороны, нормальное расслоение $\nu\left(M_{0}\right)$ размерности $2^{l}$, т.е. расслоение, возникающее при вложении $M_{0} \subset \mathbb{R}^{2^{l+1}-1}$, стабильно эквивалентно расслоению $\xi=\left(2^{l}-2 d+1\right) \varkappa$. В предположении, что число сечений этого расслоения достаточно велико (превышает $2 d$ ), удается доказать сушествование ненулевого сечения расслоения $\xi$. Это условие влечет существование ретракции порядка $d$ для многообразия $M_{0}$, а значит, и для исходного многообразия $M$. Идея доказательства изложена. 


\section{4. Вложение $S^{n}$-подобных компактов в евклидовы пространства}

В этом разделе мы докажем основные теоремы, пользуясь предложениями 1 и 2.

В категории топологических пространств обратным спектром $\left\{Y_{i} ; f_{i}\right\}$ (далее спектром) назьвается семейство топологических пространств $Y_{i}$ с индексами из направленного множества $\Lambda=\{i\}$ и семейство непрерывных отображений $f_{i}^{j}: Y_{j} \rightarrow Y_{i}$, определенных при условии $i \leqslant j$, для которых выполнены следующие условия:

$$
\begin{gathered}
f_{i}^{i}=\operatorname{Id}: Y_{i} \rightarrow Y_{i}, \quad i \in \Lambda, \\
f_{i}^{k}=f_{i}^{j} \circ f_{j}^{k}: Y_{k} \rightarrow Y_{i}, \quad i \leqslant j \leqslant k, \quad i, j, k \in \Lambda .
\end{gathered}
$$

Ниже рассматривается частный случай спектра, когда $\Lambda$ является множеством натуральных чисел. В этом случае мы пишем $f_{i}^{i+1}=f_{i+1}: Y_{i+1} \rightarrow Y_{i}$, а произвольное отображение $f_{i}^{j}, j>i$, определяется соответствующей композицией рассмотренных отображений.

Определен (обратный) предел $Y=\lim _{\leftarrow} f_{i}$ спектра $\left\{Y_{i}, f_{i}\right\}, i \in \mathbb{N}$, состоящий из всевозможных последовательностей $\left\{y_{i} \in Y_{i}\right\}$ таких, что $f_{i+1}\left(y_{i+1}\right)=y_{i}$.

Если каждое пространство $Y_{i}$ является компактным метрическим пространством с метрикой $\rho_{i}$ диаметра $\leqslant d$, то и предельное пространство $Y$ само является метрическим компактом с метрикой

$$
\operatorname{dist}(u ; v)=\sum_{i} 2^{-i} \rho_{i}\left(u_{i} ; v_{i}\right)
$$

где $\left\{u_{i}\right\}=u,\left\{v_{i}\right\}=v$.

В книге [37] доказано, что произвольный метрический компакт $X$ лебеговой размерности $n$ гомеоморфен обратному пределу $Y$ спектра $\left\{Y_{i} ; f_{i}\right\}$, состоящего из полиэдров размерности $n$ и симплициальных отображений. Следующая лемма является спецификацией этого в случае сфероподобных компактов.

ЛЕмма 4.1. Произвольный $S^{n}$-подобный компакт $X$ гомеоморфен пространству обратного предела $\lim _{\leftarrow} \alpha_{i}$ спектра $\left\{\alpha_{i}: S_{i+1}^{n} \rightarrow S_{i}^{n}\right\}, i \geqslant 1$, отображсений $n-c \oint e p$.

Доказательство изложено в [8].

ЛЕмма 4.2. Пусть $\left\{\alpha_{i}: S_{i+1}^{n} \rightarrow S_{i}^{n}\right\}, i \geqslant 1,-$ спектр отображений $n$-сфер. Пусть каждое отображение $\alpha_{i}$ дискретно реализуется относительно стандартного вложсения $S^{n} \subset \mathbb{R}^{2 n-d}, d \leqslant n / 2$. Тогда пространство $X$ обратного предела этого спектра, $X=\lim _{\leftarrow} \alpha_{i}$, вкладивается в пространство $\mathbb{R}^{2 n-d}$.

ДокАЗАТЕльство. Изложим лишш набросок доказательства. Согласно теореме Хефлигера [34] (см. также [1]) произвольное вложение $f: S^{n} \rightarrow \mathbb{R}^{2 n-d}$ при условии $d \leqslant n / 2$ изотопно стандартному. В частности, вложение $f$ является оснащенньм. Лемма доказывается аналогично следствию 5 из работы [8], где разбирается случай $d=0$. 
ДОКАЗАТЕЛЬСТВО ТЕОРЕмЫ 1. Согласно предложению 1.А каждое отображение спектра $\left\{S^{n} ; \alpha_{i}\right\}, n>4, n \neq 7$, реализуется в пространстве $\mathbb{R}^{2 n}$. Следовательно, согласно лемме 4.2 пространство $X$ допускает вложение в пространство $\mathbb{R}^{2 n}$. По лемме 4.1 произвольньй $S^{n}$-подобный компакт вкладьвается в пространство $\mathbb{R}^{2 n}$. Теорема 1 доказана.

ДОКАЗАТЕЛЬСТвО ТЕОРЕМЫ 2. В силу предложения 2 для произвольного натурального $d$ определено число $n=n(d)$ такое, что произвольное отображение $\alpha: S^{n} \rightarrow S^{n}$ дискретно (и даже изотопически) реализуется в пространстве $\mathbb{R}^{2 n-d}$. Следовательно, по лемме 4.2 пространство $X$ вкладьвается в пространство $\mathbb{R}^{2 n-d}$. По лемме 4.1 произвольный $S^{n}$-подобный компакт вкладывается в пространство $\mathbb{R}^{2 n-d}$. Теорема 2 доказана.

\section{Часть 2. Метод ортогонального проектирования в дифференциальной топологии}

\section{Введение}

Идея понижения размерности пространства, содержашего образ фигуры при ортогональном проектировании, лежит в основе геометрического построения обобшенного инварианта Хопфа и используется в первой части работы. Во второй части мы собираемся ту же идею применить для изучения диаграмм Серфа двупараметрического семейства функций, определенных на многообразии $M^{m} \times I$ со значениями на отрезке, где размерность $m$ многообразия $M^{m}$ достаточно велика. Коротко говоря, диаграммой Серфа многопараметрического семейства функций назьвается поверхность критических значений, которая рассматривается в пространстве значений+параметров. Диаграммы Серфа являются основньм инструментом при изучении гомотопического типа пространства псевдоизотопий многообразия $M^{m}$, т.е. пространства автодиффеоморфизмов многообразия $M^{m} \times I$, тождественных на нижнем основании. Для удобства мы напомним основные конструкции, связанные с понятием псевдоизотопии, полученные разными авторами в работах [17], [33], [32], [39], [40], [25], [58].

Результаты в этой части работы были доказаныпри попытке обобшить результаты В.И. Арнольда [16], полученные для функций одной переменной, на случай функций многих переменных. В этой работе В. И. Арнольда было подмечено, что конструкция Понтрягина и Тома, известная в теории кобордизмов, переносится на вешественнозначные функции. Изоморфизму Понтрягина между гомотопическими группами сфер и группами кобордизмов оснашенных многообразий соответствует изоморфизм между гомотопическими группами пространства функций на прямой с умеренными особенностями и группами кобордизма вложенных плоских кривых без горизонтальных касательных перегибов (в старших размерностях - вложенных гиперповерхностей, удовлетворяюших специальньм условиям на особенности ортогональной проекции).

Мы переходим от пространства функций на прямой или окружности к пространству функций с умеренными особенностями (обобшенными морсовскими особенностями), определенньми на гладком многообразии $M^{m} \times I$ высокой размерности. При этом мы дополнительно предполагаем, что на страте морсовских особых точек $A_{1}$ зафиксирована коориентация. Условие о коориентации является ключевым, идея определения принадлежит И. А. Володину. 
Строго говоря, идея Володина является более обшей. Она состоит в том, что при правильном определении пространства с высшими особенностями необходимо зафиксировать структурную группу, в которой лежат матрицы, отвечаюшие за примыкание стабильных сепаратрисных дисков вдоль страта фиксированного типа особенности. Нами рассмотрен первый шаг в осушествлении этой программы. Для этого мы определим пространство функций $P^{S O}\left(M^{m}\right)$, соответствующее редукции структурной группы $O$ ростка подмногообразия стабильных сепаратрисных дисков в особенностях типа "рождения-гибели" к подгрупе $S O \subset O$. Оказалось, что при выгислении гомотопических групп построенного функционального пространства $P^{S O}\left(M^{m}\right)$ естественно провести конструкцию Понтрягина в форме Уэллса. Приходится рассматривать уже не вложенные многообразия критических точек, а погруженные многообразия критических значений, т.е. диаграммы Серфа. При этом оказалось, что композиционному умножению (скобкам Тода) в стабильных гомотопических группах сфер (напоминание см. в разделе 1 ч. 1) соответствуют алгебраические соотношения, которьм удовлетворяют особенности диаграмм Серфа. Такие соотношения выявляются в рамках алгебраической К-теории. Открытая аналогия между скобками Тода и соотношениями, связанными с особенности диаграмм Серфа, может быть по аналогии продолжена на случай семейств функций с умеренными особенностями, зависящих от большего числа параметров. Это гипотеза.

Другой мотив в исследовании является методологическим и состоит в следующем. В теории псевдоизотопий (см. определение в разделе 7) введено большое число различных функциональных пространств, определение которых мы ниже напоминаем (см. диаграмму (11)). Задача вычисления гомотопического типа пространства псевдоизотопий сводится к соответствующей задаче в алгебраической К-теории. С другой стороны, вычисление гомотопического типа того или иного пространства функций с умеренными особенностями проводится методами алгебраической топологии при помоши построения соответствуюшего формального аналога как подпространства в пространстве сечений расслоения струй, см. [86]. Само же пространство псевдоизотопий не является гомотопически эквивалентньм своему формальному аналогу, см. [51], [25].

Естественно было бы выяснить, какие свойства пространства функций с той или иной структурой особенностей можно выявить при помоши методов алгебраической К-теории и сохраняются ли эти свойства при переходе от пространства функций к соответствуюшему формальному аналогу? При исследовании этого вопроса пришлось вьйти за рамки теории псевдоизотопий и развить теорию Васильева для особенностей плоской проекции погруженной замкнутой 2-поверхности в евклидово пространство. Дискриминант таких особенностей мы называем $I$-структурой. Ниже будут изучены лишш инварианты Васильева нулевого порядка в этой теории. Такие инварианты позволяют восстановить класс регулярной гомотопии (кобордизма) погруженной поверхности по структуре особенностей ее гиперпроекции. Эти формулы можно рассматривать как обобщение известной формулы Уитни, которая восстанавливает класс регулярной гомотопии плоской погруженной кривой, см., например, [15]. Для изучения инвариантов Васильева $I$-структуры порядка более чем 1 развитых методов оказывается недостаточно.

Приступим к определению необходимых понятий. Пусть $M^{m}$ - гладкое замкнутое многообразие, причем $m>6$. Обозначим через $\pi: M^{m} \times I(x) \rightarrow I(y)$ стандартную проекцию. Здесь и далее через $I(x)$ обозначен единичный отрезок числовой оси, снаб- 
женной вешественной координатой, которая обозначается через $x$. Аналогичные обозначения $I(y), I(t), I(\tau)$ также будут употребляться далее. В теории псевдоизотопий изучается пространство $H\left(M^{m}\right)$, состояшее из $C^{\infty}$-функций, заданных на многообразии $M \times I(x)$, которые принимают значения на отрезке $I(y)$, не имеют критических точек и совпадают (после отождествления $I(x)=I(y))$ с проекцией $\pi$ вблизи границы $\partial\left(M^{m} \times I(x)\right)=M^{m} \times\{0\} \cup M^{m} \times\{1\}$. Согласно конструкции Серфа [17] пространство $H\left(M^{m}\right)$ является ретрактом пространства псевдоизотопий многообразия $M^{m}$, т.е. пространства всех автодиффеоморфизмов многообразия $M^{m} \times I(x)$, тождественных в окрестности нижней границы $M \times\{0\}$.

Для изучения гомотопического типа пространства $H\left(M^{m}\right)$ в теории псевдоизотопий определяются пространства функций $P\left(M^{m}\right), P^{W}\left(M^{m}\right), P^{f r}\left(M^{m}\right)$. Подробности, необходимые для определения, будут даны в разделе 7 , ниже даются краткие определения с соответствуюшими ссылками.

Определим пространство $P\left(M^{m}\right)$ как пространство обобщенных функций Морса, т.е. функций $f: M^{m} \times I(x) \rightarrow I(y)$, совпадаюших в малой окрестности гранишы с проекцией $\pi$ и не имеющих особенностей, кроме особенностей следующих двух типов: морсовские критические точки $A_{1}$ и особые точки $A_{2}$ типа "рождения-гибели" пары морсовских особых точек соседних индексов $1 \leqslant i<i+1 \leqslant m$. Пространство $P\left(M^{m}\right)$ определяется и изучается в работе [39]. В этой работе, в частности, доказывается, что первая $m-1$ гомотопическая группа пространства $P\left(S^{m}\right), m-$ достаточно велико, описываются как группы кобордизма оснащенных многообразий $\left(N^{i} ; f r\right), i=1, \ldots, m-1$, снабженных отображениями $f: N^{i} \rightarrow B O$, причем само многообразие $N^{i}$ ограничивает как многообразие с заданным оснашением. В частности, $\pi_{1}\left(P\left(S^{m}\right)\right)=\mathbb{Z} / 2$.

Определим пространство $P^{f r}\left(M^{m}\right)$ оснашенных обобщенных морсовских функций как пространство троек $(f, \mu, \xi)$, где $f$ - обобщенная функция Морса, $\mu$-риманова метрика на $M^{m} \times I(x), \xi$ - некоторая дополнительная структура на множестве стабильных сепаратрисных дисков, восстановленных посредством $\mu$ в каждой морсовской особой точке функции. А именно, это - оснашение стабильного сепаратрисного диска, т.е. выбор базиса в касательном подпространстве к стабильному сепаратрисному диску в каждой критической точке. В случае, когда $f$ имеет особенности типа "рождения-гибели", структура $\xi$ должна быть определена на паре родившихся морсовских особых точек согласованным образом. Пространство $P^{f r}\left(M^{m}\right)$ определяется и изучается в работе [40] на языке симплициальных множеств. Оказалось, в частности, что $\pi_{i}\left(P^{f r}\left(S^{m}\right)\right)$, где $m$ достаточно велико, $m>i$, нулевые.

Пространство $P^{W}\left(M^{m}\right)$ определяется как пространство обобшенных функций Морса со следуюшей дополнительной структурой. Для каждой функции $f \in$ $P^{W}\left(M^{m}\right)$ морсовские особые точки разбиты на пары $x_{1} ; x_{2}$ точек соседних индексов, причем для каждой такой пары выделяется вложение стандартного $(m+1)$-мерного шара $U \hookrightarrow M^{m} \times I$, содержашее точки $x_{1} ; x_{2}$ и такое, что ограничение функции $f$ на этот шар задается стандартными формулами и имеет две критические точки. Указанная структура продолжается в окрестности точек "рождения-гибели", причем в каждой такой особой точке погибает или рождается пара морсовских точек соседних индексов, которые заключены внутрь одного стандартного диска. Пространство $P^{W}$, построенное в работе [25] (см. также [58]), оказьвается очень важным для изучения пространства $H\left(M^{m}\right)$. 
Наконец, пространство $F\left(M^{m}\right)$ определено как пространство всех гладких функций, совпадающих с $\pi$ в окрестности гранищы.

Мы определим новое функциональное пространство $P^{S O}\left(M^{m}\right)$ так, что задана следующая цепочка естественных отображений (все - вложения, за исключением предпоследнего $\left.i_{0}\right)$ :

$$
H\left(M^{m}\right) \stackrel{j}{\hookrightarrow} P^{W}\left(M^{m}\right) \stackrel{i_{W}}{\hookrightarrow} P^{f r}\left(M^{m}\right) \stackrel{i_{f r}}{\hookrightarrow} P^{S O}\left(M^{m}\right) \stackrel{i_{0}}{\longrightarrow} P\left(M^{m}\right) \stackrel{i}{\hookrightarrow} F\left(M^{m}\right) .
$$

Пространство $P^{S O}\left(M^{m}\right)$ определено как пространство пар $(O, f)$, где $f: M^{m} \times I \rightarrow I$ - обобщенная функция Морса, $O$ - ориентация стабильного сепаратрисного диска в каждой морсовской критической точке функции $f$. Подробнее, в окрестности морсовской критической точки $A$ можно определить систему координат $\left(a_{1}, \ldots, a_{m+1}\right)$, в которой функция $f$ определена по формуле

$$
f\left(a_{1}, \ldots, a_{m+1}\right)=\sum_{i=1}^{k} a_{i}^{2}-\sum_{i=k+1}^{m+1} a_{i}^{2} .
$$

Это канонический вид квадратичной формы, определенной на касательном пространстве $T_{A}\left(M^{m} \times I(x)\right)$ многообразия $M^{m} \times I(x)$ в точке $A$, называемой гессианом. Структура $O$ определяется как ориентация, заданная на базисе касательных векторов отрицательного подпространства гессиана. При замене системы координат рассматриваемая ориентация должна меняться инвариантным образом. Рассмотренная структура не зависит от выбора системы координат и определяет ориентацию в критической точке на ростке подмногообразия стабильного сепаратрисного диска функции $f$. При этом в окрестности критической точки типа "рождения-гибели” определенные ориентации сепаратрис для пары морсовских критических точек между собой никак не согласовьваются (о точках типа "рождения-гибели" см. раздел 7). Ниже, проявляя некоторую вольность, мы будем считать, что функции пространства $P^{S O}\left(M^{m}\right)$ могут иметь лишь критические морсовские точки пары соседних индексов $i$ и $i+1$, где $2<i<i+1<m-1$. В критических точках типа "рождения-гибели" рождается или погибает пара морсовских критических точек этих индексов. Подобное соглашение является обшепринятым в теории псевдоизотопий, поскольку в малых размерностях каждое функциональное пространство слабо гомотопически эквивалентно соответствуюшему своему подпространству, состоящему из обобщенных морсовских точек указанных индексов. В случае семейства функций с двумя параметрами, которьй для наших целей достаточен, доказательство изложено в [33].

Включения пространств в диаграмме (11) позволяют, в первую очередь, изучить свойства пространства $H\left(M^{m}\right)$. Как замечено в работе [17], очевидно, пространство $F\left(M^{m}\right)$ является стягиваемым. Игуса первым заметил, что включение пар $\left(F\left(M^{m}\right) ; H\left(M^{m}\right)\right) \hookleftarrow\left(P\left(M^{m}\right) ; H\left(M^{m}\right)\right)$ является $(m-1)$-гомотопической эквивалентностью, см., например, [58]. Это важное следствие позволяет рассматривать лишь диаграммы Серфа семейства функций с простейшими особенностями. Аналогичное свойство выполнено при замене пространства $P\left(M^{m}\right)$ на пространство $P^{W}\left(M^{m}\right)$. Более того, в работах [39], [40] определяются модели (формальные аналоги) пространств $P^{f r}\left(M^{m}\right), P^{S O}\left(M^{m}\right)$, т.е. некоторые пространства, определенные в терминах стабильной теории гомотопий, в которые исходные пространства 
естественно отображаются посредством гомотопических $(m-1)$-эквивалентностей. В работе [25] определяется формальный аналог пространства $P^{W}\left(M^{m}\right)$ как пространство ненулевых сечений кокасательного расслоения $T^{*}\left(M^{m} \times I\right)$, нигде не обращающихся в 0 и совпадающих со стандартным сечением вблизи гранищы. Естественное отображение переводит пространство $P^{W}\left(M^{m}\right)$ в это пространство сечений посредством конструкции, называемой “регуляризированный дифференциал”. Это отображение является слабой гомотопической эквивалентностью. Везде ниже, если не оговаривается противное, предполагается, что $M^{m}=S^{m}$. В этом случае конструкция формального аналога соответствуюшего функционального пространства упрошается. Переход к обшему случаю односвязного $M^{m}$ не вызывает сушественных трудностей.

В случае $\operatorname{dim}(M)=1, M^{1}=S^{1}$ или $M^{1}=\mathbb{R}^{1}$ задача изучения соответствуюших пространств сильно упрощается. В этом случае пространства $P^{f r}\left(M^{1}\right), P^{S O}\left(M^{1}\right)$ определять не приходится, поскольку все стабильные сепаратрисные диски морсовских критических точек снабжены каноническим оснащением и ориентацией. В.И. Арнольд в [16] предложил следующее полезное обобщение пространства $P\left(\mathbb{R}^{1}\right)$. Рассматривается пространство функций $f: \mathbb{R}^{1} \rightarrow \mathbb{R}^{1}$, которые совпадают с некоторой заданной функцией без особых точек вне некоторого отрезка, координаты которого не фиксируются. Говорят, что функция $f$ описанного вида принадлежит пространству $F_{k}\left(\mathbb{R}^{1}\right)$, если среди критических точек $x$ со значением $f(x)=0$ отсутствуют критические точки типа $A_{k}$. (То есть нули функции имеют кратность, не превьшаюшую $k+1$.) В [86] доказано, что $\pi_{n}\left(F_{k}\left(\mathbb{R}^{1}\right)\right)=\pi_{n-1}\left(S^{k}\right)$.

Пусть $\Lambda$ - двупараметрическое семейство функций, имеющих только обобшенные морсовские особые точки, с областью определения $S^{m} \times I(x)$, параметры которого $(t ; \tau)$ лежат в квадрате $I(t) \times I(\tau)$. В предположении, что $\Lambda$ при $(t ; \tau)=(0 ; 0)$ совпадает со стандартной проекцией $\pi$, а при $(t ; \tau) \in \partial(I(t) \times I(\tau)) \Lambda$ не имеет критических точек, семейство $\Lambda$ определяет некоторьй элемент из группы $\pi_{2}\left(P\left(S^{m}\right), H\left(S^{m}\right)\right)$. Аналогично, фиксировав выбор коориентации $O$ на стабильных сепаратрисных дисках семейства, получим семейство функций, представляющее элемент из группы $\pi_{2}\left(P^{S O}\left(S^{m}\right), H\left(S^{m}\right)\right)$. Для изучения гомотопического типа, не ограничивая обшности, можно считать, что функции семейства $\Lambda$ имеют лишш морсовские критические точки с парой соседних индексов $3 \leqslant i, i+1 \leqslant m-2$. Тогда поверхность $S$ критических значений, которая лежит (возможно, с самопересечениями) в пространстве $I(y) \times I(t) \times I(\tau)$ значений-параметров, разбивается кривой $\Sigma$ критических значений типа "рождения-гибели" на две, возможно несвязные, компоненты $S=S_{+} \cup S_{-}$. Компонента $S_{+}\left(S_{-}\right)$соответствует критическим значениям индекса $i+1(i)$. При ортогональной проекции пространства значений-параметров $I(y) \times I(t) \times I(\tau)$ на плоскость параметров $I(t) \times I(\tau)$ листы поверхности $S_{+}$и $S_{-}$проектируются без особенностей. Поверхность $S$ содержит кривую особенностей $\Sigma$, причем $S_{+}$примыкает к $\Sigma$ сверху, а $S_{-}$снизу. После малой деформации, сглаживаюшей углы вдоль $\Sigma$, поверхность превращается в погруженную поверхность $S^{\prime}$ общего положения, а проекция погруженной поверхности $S^{\prime}$ на плоскость $I(t) \times I(\tau)$ имеет особую кривую складки $\Sigma^{\prime}$, расположенную в малой окрестности кривой $\Sigma$. При этом по-прежнему кривая складки $\Sigma^{\prime}$ делит поверхность $S^{\prime}$ на компоненту $S_{+}^{\prime}$, примыкающую к кривой сверху, и компоненту $S_{-}^{\prime}$, примыкающую снизу. 
ОПрЕДЕЛЕниЕ. Скажем, что поверхность $S$, расположенная (возможно, с самопересечениями) в пространстве $I(y) \times I(t) \times I(\tau)$ и удовлетворяюшая описанным выше свойствам, моделирует диаграмму Серфа двупараметрического семейства функций, представляющего элемент из групшы $\pi_{2}\left(P^{S O}\left(S^{m}\right), H\left(S^{m}\right)\right)$.

ПРОБЛЕМА РАСПОЗНАВАНИЯ ЛОЖНЫХ ДИАГРАММ СЕРФА. Найти необходимые и достаточные условия, при которых поверхность, моделирующая диаграмму Серфа, действительно соответствует некоторой диаграмме Серфа, т.е. служит поверхностью критических значений какого-либо двупараметрического семейства функиий, представляющего әлемент $\pi_{2}\left(P^{S O}\left(S^{m}\right) ; H\left(S^{m}\right)\right)$.

Основным результатом второй части является следствие 7.2 теоремы 3 (см. раздел 7). Показано, что не любая поверхность $S$, моделирующая диаграмму Серфа, служит поверхностью критических значений. Причина состоит в следующем. Предположим, что для поверхности $S$ выполнены следующие два условия.

1. Каждая связная компонента $\Sigma_{0} \subset \Sigma$ при проекции на плоскость $I(t) \times I(\tau)$ самопересекается в четном числе точек.

2. Поверхность $S^{\prime}$ имеет ненулевой инвариант Арфа. (По поводу определения инварианта Арфа погруженной поверхности см. [31].)

В этом случае $S$ не соответствует никакой диаграмме Серфа двупараметрического семейства функций с коориентированным стратом особенностей $A_{1}$. Показано (см. следствие 7.2), что класс поверхностей, удовлетворяющих условиям 1 и 2 , не пуст.

Изложим краткое содержание ч. 2 . В разделах 5,6 развивается теория дискриминантов, что приводит к формулам (13), (15), восстанавливающим класс кобордизма погруженной поверхности $\varphi: M^{2} \rightarrow \mathbb{R}^{3}$ по особенностям проекции $\pi \circ \varphi: M^{2} \rightarrow \mathbb{R}^{3} \rightarrow$ $\mathbb{R}^{2}$ на плоскость. Для полноты изложения рассматриваются также формулы для неориентированной погруженной поверхности, хотя для доказательства основной теоремы 3 , которое завершается в разделе 8 , используется лишш ориентируемый случай. Эти результаты были получены в работе [4]. Здесь мы рассмотрим более простые доказательства формул.

В разделе 9 изложены результаты работ [6], [13]. Исследуется вопрос, при каких условиях отображение обшего положения $\varphi: M^{2} \rightarrow \mathbb{R}^{3}$ реализуется в пространстве $\mathbb{R}^{4}$ ? (Определение реализуемости см. в ч. 1.) Оказалось, что частичное препятствие к решению этой задачи связано с теоремой Рохлина о сигнатуре 4-многообразия. Попытка рассмотреть целочисленный аналог препятствия приводит к новой интерпретации первого инварианта Васильева $V_{2}$ как инварианта, обобщающего число странности (в смысле В.И. Арнольда [15]) плоской кривой общего положения.

\section{5. Формулы особенностей $I$-структуры погруженной 2 -поверхности в $\mathbb{R}^{3}$}

Под $I$-структурой ( $I=$ intersection) понимается список мультиособенностей проекции общего положения гладкого $n$-многообразия, погруженного в $(n+k)$-мерное евклидово пространство, на гиперпространство $\mathbb{R}^{n} \subset \mathbb{R}^{n+k}$. Нас будут интересовать случаи $n=2,3 ; k=1$.

В этом разделе мы развиваем технический аппарат, близкий по духу построениям работы [52]. А именно, будут перечислены особенности I-структуры, которые возникают в общем положении при $n=2, k=1$. Формулируется предложение 5.1 , согласно 
первой части которого класс кобордизма погруженной поверхности общего положения в трехмерном пространстве восстанавливается по списку особенностей $I$-структуры этой поверхности. Кроме того, дается определение так называемой пленочной поверхности, погруженной в пространство. Пленочная поверхность не является поверхностью обшего положения. Такая поверхность моделирует диаграмму Серфа двупараметрического семейства функций специального вида, так называемого канонического семейства (определение канонического семейства дается в разделе 8 , это понятие необходимо при доказательстве основной теоремы 3). Пленочная поверхность имеет особенности $I$-структуры специального вида, которые также перечисляются. Класс кобордизма пленочной поверхности (как погруженной ориентированной поверхности) восстанавливается по списку особенностей в третьей части предложения 5.1. Доказательство проводится в следуюшем разделе.

Пусть $\varphi: M^{2} \rightarrow \mathbb{R}^{3}$ - погружение, $\pi: \mathbb{R}^{3}(x, y, z) \rightarrow \mathbb{R}^{2}(x, y)$ - ортогональная проекция. Рассмотрим диаграмму мультиособенностей проекции $\pi \circ \varphi: M^{2} \rightarrow \mathbb{R}^{2}$ :

$$
\begin{array}{ccccc}
\varphi\left(M^{2}\right) & \supset & \Sigma^{1} & \supset & \Sigma^{1,1} \\
\cup & & \cup & & \\
\Delta_{2} & \supset & Q & & \\
\cup & & & & \\
\Delta_{3} & & & &
\end{array}
$$

В этой диаграмме $\Sigma^{1,1} \subset \Sigma^{1}$ - стратифицированная кривая складки с точками сборки, $\Delta_{3} \subset \Delta_{2}-$ стратифицированная кривая кратных точек погружения $\varphi, Q=$ $\Sigma^{1} \cap \Delta_{2}$. Диаграмму (12) можно рассматривать как одномерный полиэдр $K$, вложенный в пространство $\mathbb{R}^{3}$. Проекция $\pi(K)$ полиэдра на плоскость $\mathbb{R}^{2}$ имеет дополнительные изолированные особенности:

$\pi \Sigma \odot \pi \Sigma$ - точка самопересечения кривой $\pi \Sigma^{1}$;

$\pi \Sigma \odot \pi \Delta_{2}$ - точка пересечения двух ветвей кривых $\pi \Sigma^{1}$ и $\pi \Delta_{2}$, которая не получается проектированием никакой особенности на погруженной поверхности, а образуется на плоскости при проекции;

$\pi \Delta \odot \pi \Delta-$ точки самопересечения кривой $\pi \Delta_{2}$.

Для упрошения обозначений будем пользоваться сокрашениями $\pi \Sigma \Sigma, \pi \Sigma \Delta, \pi \Delta \Delta$. Иллюстрации особенностей приведены на рис. 1.

Заметим, что кривая $\pi \Sigma^{1}$ является коориентированной, причем стандартная ориентация плоскости позволяет определить на $\Sigma^{1}$ каноническую ориентацию такую, что при обходе кривой $\pi \Sigma^{1}$ в положительном направлении поверхность $\varphi(M)$ проектируется слева по направлению обхода. В соответствии с направлением вектора ориентации точки сборки делятся на два типа. В положительной точке сборки $x \in$ $\Sigma_{+}^{1,1} \subset \Sigma^{1,1}$ вектор ориентации $O(x)$ кривой складки направлен в положительном направлении оси проекции $\pi$.

Особенности $\pi Q$ и $\pi \Sigma \Sigma$ разделим на типы в соответствии со следующим правилом. Рассмотрим вектор ориентации $O(x), x \in Q$. Вектор $O(x)$ трансверсален листу поверхности $S \subset \varphi(M)$, не содержащему кривой $\Sigma$ в малой окрестности точки $x$. Пусть этот вектор в малой окрестности $U_{x}$ точки $x$ направлен в сторону полупространства $\Pi_{+}, \partial\left(\Pi_{+}\right)=S$, с границей, параллельной плоскости проекции, и содержащего положительную полуось проекции, отложенную из точки $x$. В этом случае скажем, что 


\begin{tabular}{|c|c|c|c|c|c|}
\hline$\pi \Sigma^{1,1}$ & $\pi \Delta_{3}$ & $\pi Q$ & $\pi \Sigma \Sigma$ & $\pi \Sigma \Delta$ & $\pi \Delta \Delta$ \\
\hline$B u \partial \Sigma_{+}^{1,1}$ & & $B u \partial Q_{+}$ & $B u \partial \Sigma \Sigma_{+}$ & $B u \partial_{1} \Sigma \Delta$ & \\
\hline Bud $\Sigma_{-}^{1,1}$ & & $B$ & & & \\
\hline & & $B u Q_{-}$ & $B u \partial \Sigma \Sigma_{-}$ & $B u \partial_{2} \Sigma \Delta$ & \\
\hline
\end{tabular}

Рис. 1

особенность $x$ имеет разновидность $\pi Q_{+}$. В другом случае, когда вектор направлен в направлении полупространства П_, содержащего отрицательную полуось проекции, скажем, что особенность имеет разновидность $\pi Q_{-}$. Особенность $\pi \Sigma \Sigma$ также разделим на две разновидности. К положительной разновидности $\pi \Sigma \Sigma_{+}$отнесем те особые точки, для которых упорядоченный базис (определение порядка векторов дается далее), составленный из векторов $\left(O\left(x_{1}\right) ; O\left(x_{2}\right)\right)$ ориентации к ветвям кривой $\pi \Sigma^{1}$, ориентирован положительно в плоскости $\mathbb{R}^{2}$. Для определения порядка векторы базиса выбирают так, что вектор $O\left(x_{1}\right)$ соответствует ветви кривой $\Sigma^{1}$, имеющей большую координату $z$.

Следующее определение выделяет класс погруженных поверхностей, моделируюших диаграммы Серфа двупараметрических канонических семейств обобщенных морсовских функций (определение см. в разделе 8). Коротко говоря, канонические семейства содержат лишш критические точки двух соседних индексов $i+1, i$. При этом все морсовские критические значения индекса $i+1$ лежат на интервале $] \frac{1}{2} ; 1[$, все морсовские критические значения индекса $i$ лежат на интервале $] 0 ; \frac{1}{2}[$, а все критические значения типа "рождения-гибели" равны $\frac{1}{2}$. Согласно предложению 8.1 подпространство канонических функций содержит всю необходимую информацию о гомотопическом типе пространства $P^{S O}\left(S^{m}\right)$, по крайней мере, в пределах изучаемых размерностей.

ОПРЕДЕЛЕНИЕ. Погруженная поверхность $\varphi: M^{2} \rightarrow \mathbb{R}^{3}$ называется пленочной, если кривая $\Sigma \subset M$ точек складки отображения $\pi \circ \varphi: M^{2} \rightarrow \mathbb{R}^{2}$ не содержит точек сборки и образ этой кривой при отображении $\varphi$ лежит в пересечении поверхности с плоскостью $\mathbb{R}^{2}(x, y) \subset \mathbb{R}^{3}(x, y, z), z=\frac{1}{2}$.

ЗАмЕчАниЕ. Из определения вытекает, что произвольная пленочная поверхность $M^{2}$ разделена кривой $\Sigma$ на две компоненты, вообще говоря, несвязные, обозначаемые $M_{+}$и $M_{-}$, так, что $M_{+} \bigcup_{\Sigma} M_{-}=M^{2}$. Поверхность $M_{+}\left(M_{-}\right)$расположена в положительном (отрицательном) полупространстве $R_{+}=\left\{z \geqslant \frac{1}{2}\right\}\left(R_{-}=\left\{z \leqslant \frac{1}{2}\right\}\right)$. Произвольная пленочная поверхность ориентирована. Условимся, что ориентация пленочной поверхности будет задаваться так, что вектор внешней нормали к ориентиро- 
ванной поверхности $M_{+}$направлен в положительном направлении оси проекции.

ЗАмЕчАниЕ. Естественно дается определение группы кобордизма пленочных поверхностей. Эту группу кобордизма мы будем обозначать через $\operatorname{Im} m \Sigma^{s o}(2,1)$. Операция забывания пленочной структуры на погруженной поверхности определяет гомоморфизм $\operatorname{Im} m \Sigma^{s o}(2,1) \rightarrow \operatorname{Imm}^{s o}(2,1)$. Из результатов работы [19] вытекает, что этот гомоморфизм является изоморфизмом.

Наша ближайшая задача состоит в том, чтобы исследовать особенности $I$-структуры погруженной поверхности, в частности, пленочной поверхности и найти инвариантные функции этих особенностей при регулярной гомотопии и даже при кобордизмах. Для этого определим следующие числовые характеристики особенностей.

$\sigma \in \mathbb{Z}$ определено как алгебраическое число особенностей типа $\Sigma^{1,1}$,

$$
\sigma=\operatorname{ord}\left(\Sigma_{+}^{1,1}\right)-\operatorname{ord}\left(\Sigma_{-}^{1,1}\right)
$$

$\bar{\sigma} \in \mathbb{Z}$ определено как алгебраическое число особенностей типа $\Sigma^{1,1}$, с учетом веса особенности.

Вес особенности определен ориентированным индексом проекции особенности относительно погруженной кривой проекции складки. Подробнее, пусть ind $(x)$ - целочисленньй индекс особой точки $x^{\prime}$ относительно ориентированной кривой $\pi \Sigma$, при этом точка $x^{\prime}$ получена из точки $x \in \pi \Sigma^{1,1}$ сдвигом в направлении острия точки возврата на малый вектор. Определим $\bar{\sigma}=\sum_{i} \pm \operatorname{ind}\left(x_{i}\right)$, где особенности типа $\Sigma_{+}^{1,1}$ суммируются с положительными знаками, а особенности типа $\Sigma_{-}^{1,1}$ суммируются с отрицательньми знаками.

$\bar{q} \in \mathbb{Z}$. Пусть ind $(x), x \in \pi Q,-$ индекс точки $x^{\prime}$ относительно кривой $\pi \Sigma$, где $x^{\prime}$ получена из точки $x$ сдвигом на мальй вектор в направлении коориентирующего вектора к кривой $\pi \Sigma$. Определим $\bar{q}=\sum_{i} \pm \operatorname{ind}\left(x_{i}\right)$, где особенности разновидности $\pi Q_{+}$ суммируются с положительными, а особенности разновидности $\pi Q-$ с отрицательными знаками.

$\sigma \sigma \in \mathbb{Z}$ определим как алгебраическое число особенностей типа $\pi \Sigma \Sigma$,

$$
\sigma \sigma=\operatorname{ord}\left(\pi \Sigma \Sigma_{+}\right)-\operatorname{ord}\left(\pi \Sigma \Sigma_{-}\right)
$$

$\bar{\sigma} \bar{\sigma} \in \mathbb{Z}$. Пусть $m \subset \mathbb{R}^{3}$ - прямая, параллельная оси проекции $\pi$, проходящая через точки $x_{1}, x_{2} \in \Sigma^{1}, \pi_{1}\left(x_{1}\right)=\pi_{2}\left(x_{2}\right)=x, x \in \pi \Sigma \Sigma$. Обозначим через $m_{\text {int }}$ число точек пересечения $m$ с поверхностью $\varphi\left(M^{2}\right)$, лежащих внутри отрезка $\left[x_{1} ; x_{2}\right]$, включая концевые точки. Обозначим через $m_{\text {ext }}$ число точек пересечения $m$ с поверхностью $\varphi\left(M^{2}\right)$, лежаших снаружи интервала ] $x_{1} ; x_{2}$ [, исключая концевые точки. Определим $\overline{\sigma \sigma}=\sum_{i} \pm\left[2 m_{\text {int }}\left(x_{i}\right)-m_{\text {ext }}\left(x_{i}\right)\right]$, где суммирование ведется по всем особенностям $x_{i} \in \pi \Sigma \Sigma$, причем положительный знак слагаемого выбирается для особенностей разновидности $\pi \Sigma \Sigma_{+}$и отрицательньй в случае $x_{i} \in \Sigma \Sigma_{-}$.

$\delta \delta(\bmod 2)$ определяется как четность числа особенностей типа $\pi \Delta \Delta$.

Перейдем к рассмотрению случая пленочной поверхности. По особенностям I-структуры определим следующие числа по модулю 2.

Кривая $\pi \Delta$ точек самопересечения пленочной поверхности получена как объединение двух одномерных многообразий (компонент) вдоль краев $\pi \Delta_{+,+} \cup \pi \Delta_{-,-}=\pi \Delta$ в 
соответствии с разновидностями листов поверхности, образуюших кривую самопересечения. При этом граничные точки каждой компоненты $\pi \Delta_{+,+}$и $\pi \Delta_{-,-}$совпадают с множеством точек самопересечения кривой складки $\varphi(\Sigma)$ на плоскости $\mathbb{R}^{2}(x, y)$.

$\delta \delta^{\prime}(\bmod 2)$ определим как четность числа точек самопересечения компонент $\pi \Delta_{+,+}$ и $\pi \Delta_{-},-$на плоскости проекции. Для наших дальнейших целей будет удобно ввести обозначения (которые будут использоваться при доказательстве предложения 5.1) $\left(\delta_{++} ; \delta_{++}\right)$и $\left(\delta_{--} ; \delta_{--}\right)$для четности чисел точек самопересечения кривых $\pi \Delta_{+,+}$ и $\pi \Delta_{-,-}$соответственно. Теперь число $\delta \delta^{\prime}(\bmod 2)$ можно определить по формуле $\delta \delta^{\prime}=\left(\delta_{++} ; \delta_{++}\right)+\left(\delta_{--} ; \delta_{--}\right)$. Отсюда, в частности, можно заметить, что при подсчете числа $\delta \delta$ значение $\left(\delta_{++} ; \delta_{--}\right.$) (которое определяется как четность числа точек пересечения кривых $\pi \Delta_{+,+}$и $\Delta_{-,-}$, исключая граничные точки этих компонент) не учитывается.

$i \sigma \sigma(\bmod 2)$. Пусть $x \in \pi \Sigma \Sigma$. Определим $\operatorname{Ind}(x)$ как индекс точки $x^{\prime}$ относительно кривой $\pi \Delta$, причем точка $x^{\prime}$ получена из точки $x$ в результате сдвига на мальй вектор по направлению острия сборки на кривой $\pi \Delta$, две ветви которой, $\pi \Delta_{+,+}$и $\pi \Delta_{-,-}$, примыкают к особой точке $\pi \Sigma \Sigma$. Определим $i \sigma \sigma=\sum_{i} \operatorname{Ind}\left(x_{i}\right)$, где суммирование ведется по всем особым точкам множества $\pi \Sigma \Sigma$.

ПРЕДЛОЖЕНИЕ 5.1. Пусть $\varphi: M^{2} \rightarrow \mathbb{R}^{3}$ - погружение общего положсения замкнутой, вообще говоря, неориентированной поверхности.

1. Класс кобордизма погружения $\varphi$ в группе $\operatorname{Imm}^{\circ}(2,1)=\mathbb{Z} / 8$ определяется особенностями I-структуры по формуле

$$
G(\varphi)=\sigma+2 \sigma \sigma+4 \delta \delta \quad(\bmod 8)
$$

2. Справедлива челочисленная формула

$$
\bar{\sigma}+2 \overline{\sigma \sigma}+\bar{q}=0
$$

3. Пусть $\psi$ - пленочная поверхность. Класс кобордизма $\psi$ в группе $\operatorname{Imm}^{\text {so }}(2,1)=\mathbb{Z} / 2$ определяется по особенностям I-структуры по формуле

$$
H(\psi)=\delta \delta^{\prime}+i \sigma \sigma \quad(\bmod 2)
$$

ЗАмЕчАниЕ. Используя алгебраическую конструкцию инварианта Арфа, определенного для некоторой специальной квадратичной формы, заданной на группе гомологий неориентированных 1-циклов поверхности, погружение которой изучается, можно записать $H(\psi)=\operatorname{Arf}(\psi)$. Аналогично, в случае неориентированной поверхности значение $G(\varphi)$ можно приравнять к обобшенному инварианту Брауна $\mathbb{Z} / 4$-значной квадратичной формы, определяемой на той же группе. Соответствующие конструкции таких форм и определения инвариантов Арфа и Брауна изложены в [31].

ЗАмечАниЕ. Формула (13) может быть обобщена на произвольные размерности для численного выражения Arf-инварианта (сигнатуры) погруженного замкнутого ориентированного многообразия $M^{2 k} \rightarrow \mathbb{R}^{3 k}, k>1$. Формула (14) представляется интересной в связи с общей проблематикой работы [16]. Основной результат в этой части обзора (теорема 3) доказан при помощи формулы (15). 
ПрИмеР КонстАнтиновА. Построим пример пленочной поверхности $\psi: T^{2} \rightarrow$ $\mathbb{R}^{3}$, назьваемой тором Константинова. Эта поверхность является основным геометрическим объектом исследования в ч. 2. Она используется в следствии 7.3 основной теоремы 3 , а также в разделе 9 , пример 9.8 .

НЕФОРМАЛЬНОЕ ЗАМЕЧАНИЕ. По Поводу названия "Тор Константинова" сообщу, что в 1979 году Николай Николаевич Константинов рассказал мне об удивительной кривой на плоскости, которая была им открыта в 1961 году (подтверждением является доклад на семинаре Л. В. Келдьш и неопубликованная рукопись, датированная этим годом). Эта кривая была впоследствии независимо переоткрыта Дж. Милнором; ее рисунок можно найти, например, в книге [30; рис. 1]. Кривая Милнора доставляет нетривиальньй пример пленочной поверхности. Тем не менее, мне не удалось выявить никаких интересных свойств этой пленочной поверхности в связи с проблемой распознавания ложных диаграмм Серфа. Пример пленочного тора, которьй строится ниже, является вариацией на тему, заданной кривой Милнора. Ряд интересных свойств этого тора выявляется следствием 7.3 теоремы 3 и далее, в последнем разделе второй части, который посвящен проблеме реализации отображений 2-поверхностей в 4-пространстве.

Пусть $\Pi_{i}, i=1,2,3$, три двумерных поверхности с краем, причем $\Pi_{1} \approx D^{2} ; \Pi_{2} \approx$ $S^{1} \times I ; \Pi_{3} \approx T^{2} \backslash D^{2}$. Поверхность тора получается в результате отождествления компонент $\Pi_{1} ; \Pi_{2}$ вдоль обшей гранишы $\Sigma_{0}$ и компонент $\Pi_{2}$ и $\Pi_{3}$ вдоль обшей границы $\Sigma_{1}$. Отображение $\psi$ будет построено так, что при проекции $\pi: \mathbb{R}^{3} \rightarrow \mathbb{R}^{2}$ кривые $\Sigma_{0}$ и $\Sigma_{1}$ являются кривыми складки и не содержат точек сборки.

Опишем погружение $g_{3}=\left.\pi \circ \psi\right|_{\Pi_{3}}: \Pi_{3} \rightarrow \mathbb{R}^{2}$. Рассмотрим на рис. 2 криволинейньй плоский шестиугольник $k=A B C D E F$.

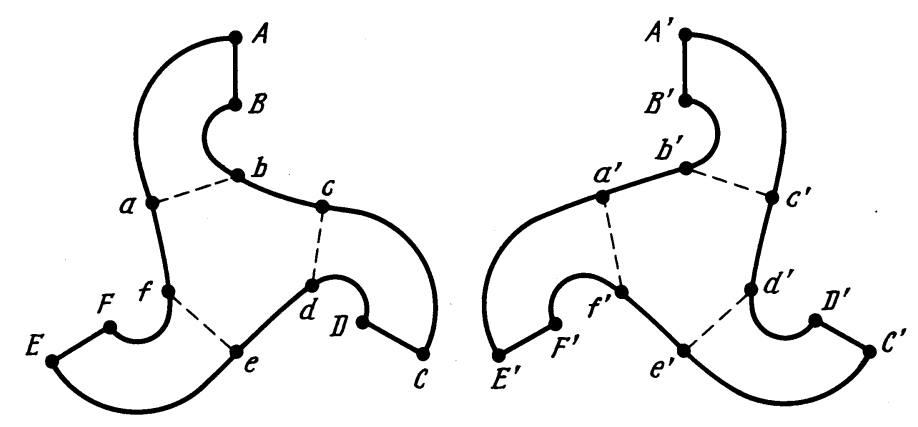

Рис. 2

Второй экземпляр $k^{\prime}=A^{\prime} B^{\prime} C^{\prime} D^{\prime} E^{\prime} F^{\prime}$ шестиугольника выбираем симметричньм первому. Определена поверхность $\Pi_{3}=k \bigcup_{\partial} k^{\prime}$, при этом пары отрезков $A B, A^{\prime} B^{\prime}$, $C D, C^{\prime} D^{\prime}, E F, E^{\prime} F^{\prime}$ на гранищах шестиугольников отождествляются между собой. Построим погружение $g_{3}: \Pi_{3} \rightarrow \mathbb{R}^{2}$ так, что $g_{3}(k)$ пересекается с $g_{3}\left(k^{\prime}\right)$ по шестиугольнику $a b c d e f$. Образ погружения $g$ ограничен кривой $\pi \Sigma_{1}=g\left(\partial \Pi_{3}\right)$. Опишем погружение $g_{2}=\left.\pi \circ \psi\right|_{\Pi_{2}}: \Pi_{2} \rightarrow \mathbb{R}^{2}$ в соответствии с рис. 3 . 


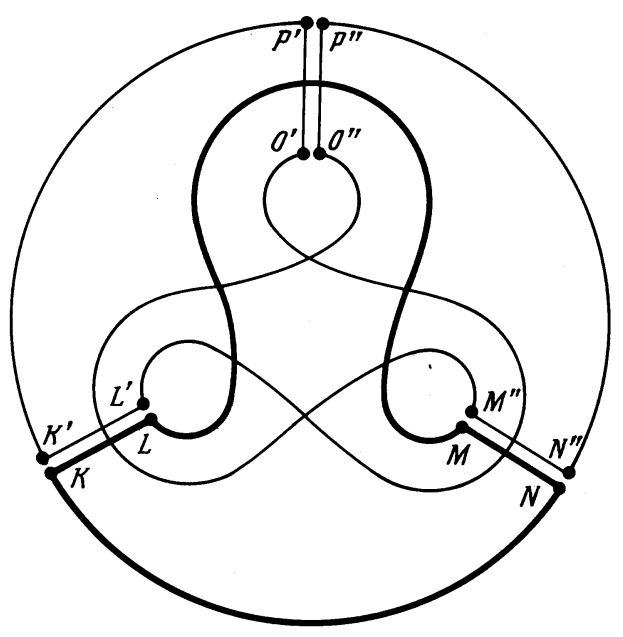

Рис. 3

Рассмотрим криволинейный четырехугольник $l=K L M N$, изображенный жирной линией. Еще два экземпляра четырехугольника, конгруэнтные исходному, обозначим через $l^{\prime}=K^{\prime} L^{\prime} O^{\prime} P^{\prime}$ и $l^{\prime \prime}=K^{\prime \prime} L^{\prime \prime} M^{\prime \prime} N^{\prime \prime}$. Отождествим соответствующие отрезки в четырехугольниках $l, l^{\prime}, l^{\prime \prime}$, помеченные одинаковыми буквами. В результате отождествления получим цилиндр $L \bigcup_{\partial} L^{\prime} \bigcup_{\partial} L^{\prime \prime}=\Pi_{2}$, ограниченньй кривыми $\Sigma_{0}, \Sigma_{1}$. Этот цилиндр, погруженньй в плоскость посредством отображения $g_{2}$, осуществляет гомологию кривых $\pi \Sigma_{0}$ и $\pi \Sigma_{1}$, изображенных на рис. 4.

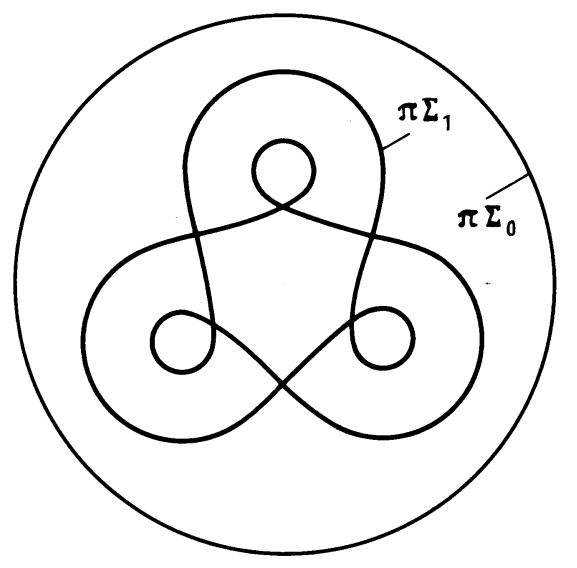

Рис. 4

Погружение $g_{1}: \Pi_{1} \rightarrow \mathbb{R}^{2}$ параметризует диск с граничной кривой $\pi \Sigma_{0}$. Отождествив три построенные поверхности в соответствии с указанным выше правилом, получим проекцию тора $g: T^{2} \rightarrow \mathbb{R}^{2}$, имеюшую лишш особенности складки на кривых $\Sigma_{0}$, $\Sigma_{1}$. Чтобы построить погружение $\psi$ такое, что $\pi \circ \psi=g$, достаточно погрузить эти кривые в плоскость, части поверхности $\Pi_{1}, \Pi_{3}$ погрузить в верхнюю полуплоскость с 
границей $\mathbb{R}^{2}$, а поверхность $\Pi_{2}$ погрузить в нижнюю полуплоскость посредством задания подходяших функций $\rho_{i}, i=1,2,3$, высоты вдоль оси проекции. При этом функцию высоты следует выбирать тождественно равной 0 на $\Sigma_{j}, j=1,2$, положительной на $\Pi_{1} \backslash \partial \Pi_{1}$ и отрицательной на $\Pi_{2} \backslash \partial \Pi_{2}$. Построение примера закончено.

Изучим $I$-структуру погруженной пленочной поверхности Константинова. Удобно воспользоваться методом "шляпных диаграмм", описанным в книге [27]. Заметим, что “шляпные диаграммы” и $I$-структура поверхности зависят от выбора функций высоты $\rho_{i}$. Для некоторого выбора этих функций $I$-структура пленочной поверхности Константинова изображена на рис. 5 .

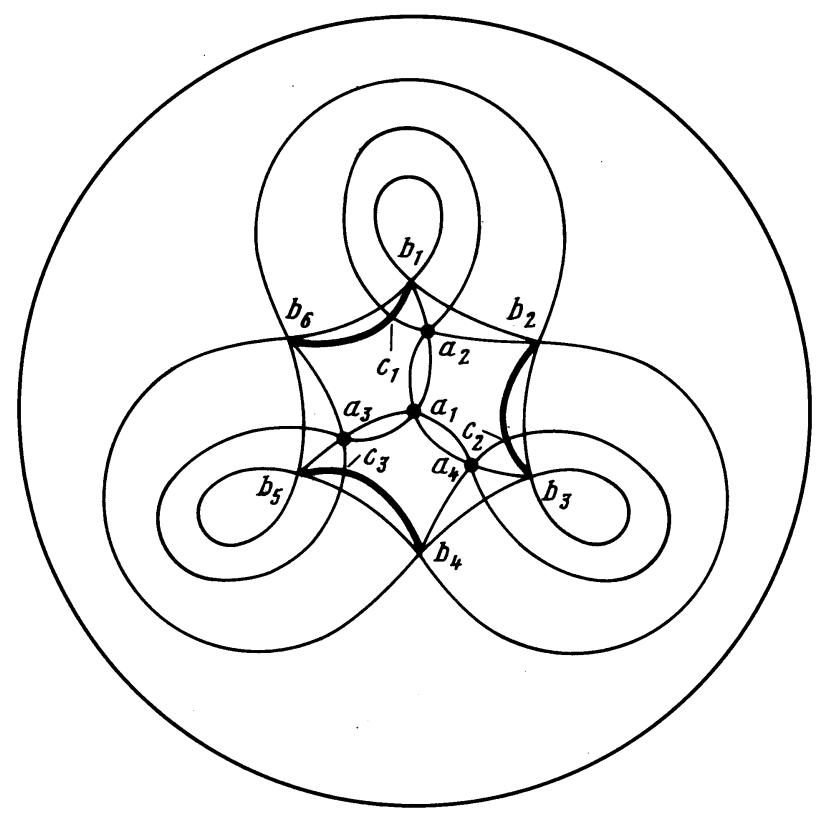

Рис. 5

Заметим, что функции $\rho_{i}$ выбраны так, что поверхность Константинова симметрична относительно поворота на угол $2 \pi / 3$ относительно оси, проходящей через центральную точку $a_{1}$ типа $\pi \Delta_{3}$ и перпендикулярной плоскости проекции. Отрезки линии самопересечения изображены на рисунке линиями различной толщины. Три отрезка $\left[b_{6}, b_{1}\right],\left[b_{2}, b_{3}\right],\left[b_{4}, b_{5}\right]$, изображенные жирной линией, соответствуют кривой $\pi \Delta_{+}$и образованы в результате самопересечения поверхности $\psi\left(\Pi_{3}\right)$. Точки $b_{i}, i=1, \ldots, 6$, на концах этих отрезков имеют особенность типа $\pi \Sigma \Sigma$. Участки линии самопересечения, помеченные тонкой линией, соответствуют кривой $\pi \Delta_{-}$и образованы самопересечением поверхности $\psi\left(\Pi_{2}\right)$. Точки самопересечения кривых $\pi \Delta_{+}$и $\pi \Delta_{-}$обозначены на рисунке через $c_{i}, i=1,2,3$.

Рассмотрим малую деформацию общего положения $\psi \rightarrow \varphi$, где $I$-структура поверхности $\varphi\left(T^{2}\right)$ изображена на рис. 6. 


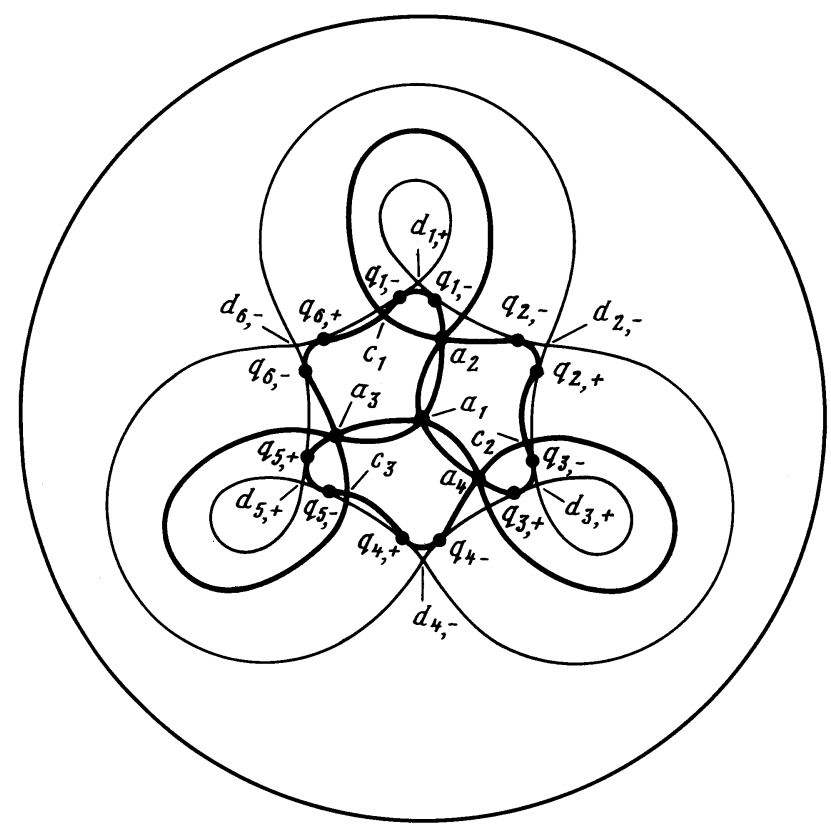

Рис. 6

Важно проследить за деформацией в окрестности точек типа $\pi \Sigma \Sigma$. Выбор деформации определяется типами точек, помеченных на рис. 6. При этом каждый касп $b_{i}$ деформируется в пару точек $q_{i,+}, q_{i,-}$ типа $\pi Q$ противоположных знаков. Вне окрестности указанных особенностей $I$-структура поверхности не изменяется.

Вычислим значения слагаемых в формулах (12)-(14) на примере погружения Константинова. Очевидны равенства $\sigma(\varphi)=\bar{\sigma}(\varphi)=0$. Далее,

$$
\begin{gathered}
\sigma \sigma\left(d_{i,+}\right)=1, \quad \sigma \sigma\left(d_{i,-}\right)=-1, \quad i=1, \ldots, 6 ; \quad \delta\left(c_{j}\right)=1, \quad j=1,2,3 ; \\
m_{\mathrm{ext}}\left(d_{i,+}\right)=m_{\mathrm{ext}}\left(d_{i,-}\right)=0, \quad m_{\mathrm{int}}\left(d_{i,+}\right)=m_{\mathrm{int}}\left(d_{i,-}\right)=2 ; \\
\overline{\sigma \sigma}=\sum_{i}\left[2 m_{\mathrm{int}}\left(d_{i,+}\right)-m_{\mathrm{ext}}\left(d_{i,+}\right)\right]-\left[2 m_{\mathrm{int}}\left(d_{i,-}\right)-m_{\mathrm{ext}}\left(d_{i,-}\right)\right]=0 ; \\
\bar{q}\left(q_{i,+}\right)=+1, \quad \bar{q}\left(q_{i,-}\right)=-1 ; \quad\left(\delta_{++}, \delta_{++}\right)=\left(\delta_{--}, \delta_{--}\right)=0 ; \\
\operatorname{Ind}\left(b_{2 j}\right)=0, \quad \operatorname{Ind}\left(b_{2 j-1}\right)=1, \quad i \sigma \sigma=\sum_{j} \operatorname{Ind}\left(b_{2 j-1}\right)=1(\bmod 2), \quad j=1,2,3 ; \\
\left(\delta_{+,+}, \delta_{-,-}\right)=1 ; \quad \delta \delta=1, \quad \delta \delta^{\prime}=0 .
\end{gathered}
$$

При подстановке значений в формулы (13) и (15) получаем

$$
G(\varphi)=4 \quad(\bmod 8), \quad H(\psi)=1 \quad(\bmod 2)
$$

ЗАмЕчАниЕ. Формула (14) содержит ненулевые слагаемые для поверхностей Боя и для погруженной сферы, двулистно накрьвающей эту поверхность. Эти вычисления можно найти в [4]. 


\section{6. Особенности $I$-структуры погруженного 3 -многообразия в $\mathbb{R}^{4}$}

В этом разделе будет доказано предложение 5.1. Пусть $N^{3}$ - гладкое трехмерное многообразие с краем $\partial\left(N^{3}\right)=M^{2}, \psi:\left(N^{3}, M^{2}\right) \rightarrow\left(\mathbb{R}^{3} \times[0 ;+\infty), \mathbb{R}^{3} \times\{0\}\right)$ - погружение общего положения этого многообразия в 4-мерное евклидово пространство, $\pi: \mathbb{R}^{3} \times[0 ;+\infty) \rightarrow \mathbb{R}^{2} \times[0 ;+\infty)$ - стандартная ортогональная проекция. Если край не пуст, предположим, что особенности $I$-структуры проекции $\pi$, ограниченные на край $M^{2}$, находятся в общем положении. Мультиособенности проекции $\pi \circ \psi: N^{3} \rightarrow \mathbb{R}^{2} \times[0 ;+\infty)$ включены в следуюшую диаграмму:

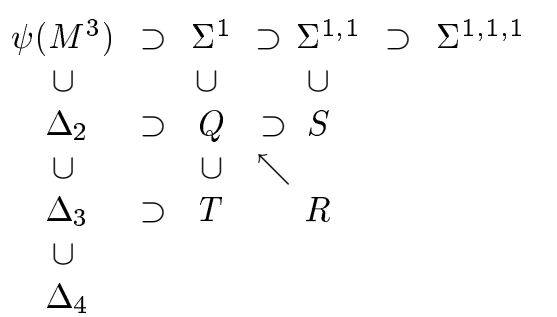

В этой диаграмме $\Sigma^{1,1,1} \subset \Sigma^{1,1} \subset \Sigma^{1}$ - стратифицированная поверхность сборки, содержашая кривые складки и изолированные особенности типа "ласточкин хвост", $\Delta_{4} \subset \Delta_{3} \subset \Delta_{2}-$ стратифипшированная поверхность кратных точек погружения $\psi$, причем точки кратности 3 образуют кривые $\Delta_{3}$, вдоль которых поверхность $\Delta_{2}$ самопересекается с кратностью 3 , точки $\Delta_{4}$ кратности 4 образуют конечное множество, причем в окрестности каждой точки пересекаются 6 листов поверхности $\Delta_{2}$ и 4 ветви кривой $\Delta_{3}$. Поверхности $\Sigma^{1}$ и $\Delta_{2}$ на многообразии $\psi\left(N^{3}\right)$ пересекаются вдоль кривой $Q$. Конечное множество особенностей типа $T$ состоит из точек пересечения кривой $\Delta_{3}$ и поверхности $\Sigma^{1}$ на погруженном многообразии $\psi\left(M^{3}\right)$. Аналогично, конечное множество $S$ образовано пересечением кривой $\Sigma^{1,1}$ и поверхности $\Delta_{2}$. Конечное множество особенностей типа $R$ образовано в результате самопересечения поверхности $\psi\left(\Sigma^{1}\right)$ в пространстве $\mathbb{R}^{4}$. Другим способом особые точки типа $R$ могут быть определены как критические точки проекции $\pi: \Delta_{2} \rightarrow \mathbb{R}^{3}$. Особенности, определенные диаграммой (16), образуют двумерный полиэдр специального вида, причем проекция этого полиэдра в $\mathbb{R}^{3}$ имеет особенности самопересечения общего положения. Проекцию этого полиэдра будем называть полиэдром $I$-структуры погружения $\psi$. Тем самым, после введения очевидных обозначений возникают следующие три типа особых кривых, лежащих в пространстве $\mathbb{R}^{3}$ :

$$
\pi \Delta_{2} \odot \pi \Sigma^{1}, \quad \pi \Delta_{2} \odot \pi \Delta_{2}, \quad \pi \Delta_{2} \odot \pi \Sigma^{1},
$$

и 10 типов изолированных особых точек:

$$
\begin{gathered}
\pi \Delta_{2} \odot \pi \Delta_{2} \odot \pi \Delta_{2}, \quad \pi \Delta_{2} \odot \pi \Delta_{2} \odot \pi \Sigma^{1}, \quad \pi \Delta_{2} \odot \pi \Sigma^{1} \odot \pi \Sigma^{1}, \\
\pi \Sigma^{1} \odot \pi \Sigma^{1} \odot \pi \Sigma^{1}, \quad \pi \Delta_{3} \odot \pi \Delta_{2}, \quad \pi \Delta_{3} \odot \pi \Sigma^{1}, \\
\pi Q \odot \pi \Delta_{2}, \quad \pi Q \odot \pi \Sigma^{1}, \quad \pi \Sigma^{1,1} \odot \pi \Sigma^{1}, \quad \pi \Sigma^{1,1} \odot \pi \Delta_{2} .
\end{gathered}
$$

Заметим, например, что множество особенностей $\pi \Delta_{2} \odot \pi \Sigma^{1}$ не совпадает, вообще говоря, с множеством $\pi \Delta_{2} \cap \pi \Sigma^{1}$, так как к первому относятся не все точки пересечения указанных кривых на плоскости, а лишь те, которым соответствуют различные 
прообразы при проектировании. Для сокращения обозначений будем использовать символы $\pi \Delta \Delta, \pi \Sigma \Sigma, \pi \Sigma \Delta$ не только для изолированых особенностей $I$-структуры для $n=2$ в диаграмме (12), но и для соответствующих ветвей особых кривых в диаграмме (16) при $n=3$.

Согласно мультиструйному аналогу теоремы Тома о трансверсальности множество погружений, имеющих только описанные особенности $I$-структуры, образует открытое и всюду плотное подпространство в пространстве всех погружений $\operatorname{Imm}\left(N^{3} ; \mathbb{R}^{4}\right)$.

ДоКАЗАТЕльСТво формУЛЫ (13). Приступим к доказательству формул предложения 5.1. Докажем, что выражение $G(\varphi)$ является инвариантом кобордизма. Пусть $\varphi_{i}: M_{i} \rightarrow \mathbb{R}^{3} ; i=1,2$, погружения, $\psi: N^{3} \rightarrow \mathbb{R}^{3} \times I$ - кобордизм, связывающий погружения $\varphi_{1}, \varphi_{2}$, такой, что $\partial N^{3}=M_{1} \cup M_{2} ;\left.\psi\right|_{M_{1}}=\varphi_{1} ;\left.\psi\right|_{M_{2}}=\varphi_{2}$. Особенности $I$-структуры погружений $\varphi_{1}, \varphi_{2}$ связаны кобордизмом особенностей $I$-структуры погружения $\psi$. Это означает, что полиэдры вида (16) особенностей $I$-структуры погружений $\varphi_{1}, \varphi_{2}$ ограничивают двумерный полиэдр особенностей $I$-структуры погружения $\psi$, заданный диаграммой вида (15). Рассмотрим список изолированных особых точек $I$-структуры погружения $\psi$, примыкающих к особой кривой $\pi \Sigma^{1} \odot \pi \Sigma^{1}$ :

$$
\pi R, \quad \pi \Sigma^{1} \odot \pi \Sigma^{1} \odot \pi \Sigma^{1}, \quad \pi \Sigma^{1} \odot \pi \Sigma^{1} \odot \pi \Delta_{2}, \quad \pi Q \odot \pi \Sigma^{1} .
$$

Следуюший список составляют изолированные особенности, содержащие в малой своей окрестности особые кривые $\pi \Delta_{2} \odot \pi \Delta_{2}$ :

$$
\pi T, \quad \pi R, \quad \pi \Delta_{2} \odot \pi \Delta_{2} \odot \pi \Delta_{2}, \quad \pi \Delta_{2} \odot \pi \Delta_{2} \odot \pi \Sigma^{1}, \quad \pi Q \odot \pi \Delta_{2} .
$$

Следуюший список составляют изолированные особенности, содержашие в малой своей окрестности кривые $\pi Q$ :

$$
\pi T, \quad \pi R, \quad \pi S, \quad \pi Q \odot \pi \Sigma^{1}, \quad \pi Q \odot \pi \Delta_{2} .
$$

Выражение $G$ и каждое слагаемое $\sigma, \sigma \sigma$ и $\delta \delta$ определяют одномерные коцепи на 1 -остове полиэдра $I$-структуры. Проверка инвариантности выражения $G$ при кобордизмах состоит в том, что мы доказьваем, что коцеп $G$ является коциклом по модулю 8. Аналогичное утверждение ранее использовалось в [84].

Среди перечисленных особенностей имеются три, в окрестности которых коцеп $G$ имеет особенность, связанную с примыканием 1-ребер с носителем $G$ различных типов, а именно:

$$
\pi \Sigma^{1,1,1}, \quad \pi R, \quad \pi \Sigma^{1,1} \odot \pi \Sigma^{1} .
$$

Рассмотрим каждую особенность по отдельности.

К особой точке $\pi \Sigma^{1,1,1}$ примыкают два ребра $\alpha, \alpha^{\prime}$ особенности $\pi \Sigma^{1,1}$ и одно ребро $\gamma \subset \pi \Sigma^{1} \odot \pi \Sigma^{1}$. Легко проверяется тождество:

$$
\sigma(\alpha)+\sigma\left(\alpha^{\prime}\right)+2 \sigma \sigma(\gamma)=0
$$

где слагаемые выражения обозначают значения коцепей на соответствующих 1-симплексах. Здесь и далее проверка этого и аналогичных тождеств происходит в соответствии с изложением [2]. 
$\mathrm{K}$ особенности $\pi R$ примыкают две пары вертикальных ребер $\pi \Sigma^{1} \odot \Sigma^{1}$, обозначаемых через $\left(\alpha_{1,1}, \alpha_{1,2}\right),\left(\alpha_{2,1}, \alpha_{2,2}\right)$, и ребро $\varepsilon \in \pi \Delta \Delta$. Справедливо равенство:

$$
2 \sigma \sigma\left(\alpha_{1,1}\right)+2 \sigma \sigma\left(\alpha_{2,1}\right)+4 \delta \delta(\varepsilon)=2 \sigma \sigma\left(\alpha_{1,2}\right)+2 \sigma \sigma\left(\alpha_{2,2}\right) .
$$

Заметим, что коцепь $\delta \delta$ не является целочисленной и равенство следует понимать по модулю 8.

К особенности $\pi \Sigma^{1,1} \odot \pi \Sigma^{1}$ примыкает пара вертикальных ребер $\alpha_{1}, \alpha_{2}$ типа $\pi \Sigma^{1,1}$ и пара ребер $\gamma, \gamma^{\prime} \in \pi \Sigma^{1} \odot \pi \Sigma^{1}$. Справедливы равенства:

$$
\sigma \sigma(\gamma)+\sigma \sigma\left(\gamma^{\prime}\right)=0, \quad \sigma\left(\alpha_{1}\right)=\sigma\left(\alpha_{2}\right) .
$$

Проведенные вычисления доказывают, что $G\left(\varphi_{1}\right)=G\left(\varphi_{2}\right)$, если $\varphi_{1}$ и $\varphi_{2}$ лежат в одном классе кобордизма группы $\operatorname{Imm}^{o}(2,1)$. В соответствии с вычислением значения $G$ из раздела 5 для погружения Константинова $\varphi: T^{2} \rightarrow \mathbb{R}^{3}$ имеем $G(\varphi)=4$ $(\bmod 8)$. По предложению 1.1 гомоморфизм $I: \operatorname{Imm}^{s o}(2,1) \rightarrow \operatorname{Imm}^{o}(2,1)$ является мономорфизмом. Поскольку гомоморфизм $G \circ I: \operatorname{Imm}^{s o}(2,1) \rightarrow \mathbb{Z} / 8$ является мономорфизмом, отображение $G: \operatorname{Imm}^{\circ}(2,1) \rightarrow \mathbb{Z} / 8$ также является мономорфизмом. Формула (13) доказана.

ДоКАЗАТЕЛЬСТвО ФОРМУЛЫ (14). Проверим, что коцеп $B$ является целочисленным коциклом. Перечислим изолированные особенности $I$-структуры, в окрестности которых имеются 1-симплексы с носителем $B$ :

$$
\begin{gathered}
\pi \Sigma^{1,1,1}, \quad \pi S, \quad \pi T, \quad \pi R, \\
\pi \Sigma^{1,1} \odot \pi \Sigma^{1}, \quad \pi \Sigma^{1} \odot \pi \Sigma^{1} \odot \pi \Sigma^{1}, \quad \pi Q \odot \pi \Sigma^{1} .
\end{gathered}
$$

К особой точке $\pi \Sigma^{1,1,1}$ примыкают два ребра $\alpha, \alpha^{\prime} \subset \pi \Sigma^{1,1}$ и ребро $\gamma \subset \pi \Sigma^{1} \odot \pi \Sigma^{1}$. Справедливо равенство:

$$
\bar{\sigma}(\alpha)=\bar{\sigma}\left(\alpha^{\prime}\right)=-2\left[-m_{\text {ext }}(\gamma)+2 m_{\text {int }}(\gamma)\right] .
$$

$\mathrm{K}$ особой точке $\pi S$ примыкает пара вертикальных ребер $\beta_{1}, \beta_{2} \subset \pi Q$ и пара вертикальных ребер $\alpha_{1}, \alpha_{2} \subset \pi \Sigma^{1,1}$. Имеем $\bar{q}\left(\beta_{1}\right)=\bar{q}\left(\beta_{2}\right), \bar{\sigma}\left(\alpha_{1}\right)=\bar{\sigma}\left(\alpha_{2}\right)$.

К особой точке $\pi T$ примыкают две пары $\beta_{i, 1}, \beta_{i, 2}, i=1,2$, вертикальных ребер $\pi Q$, пара вертикальных ребер $\pi \Delta_{3}$ и две пары вертикальных ребер $\varepsilon_{i, 1}, \varepsilon_{i, 2} \subset \pi \Sigma^{1} \odot \pi \Delta_{2}$. Справедливо равенство $\bar{q}\left(\beta_{i, 1}\right)=\bar{q}\left(\beta_{i, 2}\right)$.

$\mathrm{K}$ особой точке типа $\pi R$ примыкают пара вертикальных ребер $\gamma_{1}, \gamma_{2} \subset \pi \Sigma^{1} \odot \pi \Sigma^{1}$, две пары вертикальных ребер $\beta_{i, 1}, \beta_{i, 2}, i=1,2$, типа $\pi Q$ и одно ребро $\pi \Delta_{3}$. Справедливо равенство:

$$
\overline{\sigma \sigma}\left(\gamma_{1}\right)+\bar{q}\left(\beta_{1,1}\right)+\bar{q}\left(\beta_{2,1}\right)=\overline{\sigma \sigma}\left(\gamma_{2}\right)+\bar{q}\left(\beta_{1,2}\right)+\bar{q}\left(\beta_{2,2}\right) .
$$

К особой точке типа $\pi \Sigma^{1,1} \odot \pi \Sigma^{1}$ примыкают два ребра $\gamma, \gamma^{\prime} \subset \pi \Sigma^{1} \odot \pi \Sigma^{1}$ и пара вертикальных ребер $\alpha_{1}, \alpha_{2} \subset \pi \Sigma^{1,1}$. Справедливо тождество:

$$
\overline{\sigma \sigma}(\gamma)+\overline{\sigma \sigma}\left(\gamma^{\prime}\right)+\bar{\sigma}\left(\alpha_{1}\right)=\bar{\sigma}\left(\alpha_{2}\right) .
$$


К особой точке типа $\pi \Sigma^{1} \odot \pi \Sigma^{1} \odot \pi \Sigma^{1}$ примыкают три пары вертикальных ребер $\gamma_{i, 1}, \gamma_{i, 2}, i=1,2,3$, типа $\pi \Sigma^{1} \odot \pi \Sigma^{1}$. Справедливо равенство:

$$
\sum_{i} \overline{\sigma \sigma}\left(\gamma_{i, 1}\right)=\sum_{i} \overline{\sigma \sigma}\left(\gamma_{i, 2}\right)
$$

(Заметим, что $\overline{\sigma \sigma}\left(\gamma_{i, 1}\right) \neq \overline{\sigma \sigma}\left(\gamma_{i, 2}\right)$.)

К особой точке $\pi Q \odot \pi \Sigma^{1}$ примыкают пара вертикальных ребер $\beta_{1}, \beta_{2}$ особой кривой $\pi Q$, пара $\alpha_{1}, \alpha_{2}$ вертикальных ребер особой кривой $\pi \Sigma^{1} \odot \pi \Sigma^{1}$ и пара вертикальных ребер особой кривой $\pi \Sigma^{1} \odot \pi \Delta_{2}$. Справедливо равенство

$$
\bar{q}\left(\beta_{1}\right)+\overline{\sigma \sigma}\left(\alpha_{1}\right)=\bar{q}\left(\beta_{2}\right)+\overline{\sigma \sigma}\left(\beta_{2}\right) .
$$

Проведенные вычисления доказывают, что для погружений $\varphi_{1}, \varphi_{2}$, лежаших в одном классе кобордизма $\operatorname{Im}^{o}(2,1)$, справедливо равенство $B\left(\varphi_{1}\right)=B\left(\varphi_{2}\right)$. По теореме 1.1 имеем $\operatorname{Im}^{o}(2,1) \otimes Q=0$. Формула (14) доказана.

ДоКАЗАТЕльСТво фОРмУлы (15). Определим группу $\operatorname{Im} m \Sigma^{s o}(2,1)$ как одну из разновидностей групшы кобордизмов погружений, следуя построениям работы [19]. Пусть $\varphi: M^{2} \rightarrow \mathbb{R}^{3}$ - погружение ориентированной поверхности $M^{2}$. Скажем, что погружение $\varphi$ имеет регулярную складку, если проекция $\pi \circ \varphi: M^{2} \rightarrow \mathbb{R}^{2}$ не имеет точек сборки и вектор внешней нормали к поверхности в точках кривых складки $\Sigma^{1} \subset M^{2}$ проектируется в коориентируюший вектор к кривой $\pi \Sigma$, т.е. внешним образом по отношению к проекции поверхности. Определено разбиение $M=M_{+} \bigcap_{\Sigma} M_{-}$ в соответствии с направлениями векторов внешней нормали вдоль оси проекции.

Естественным образом определяется отношение кобордантности на множестве погружений с регулярными складками. Скажем, что два погружения $\varphi_{1}, \varphi_{2}$ кобордантны в группе $\operatorname{Imm} \Sigma^{s o}(2,1)$, если кобордизм $\psi: N \rightarrow \mathbb{R}^{3} \times I$, связываюший построенные погружения в групе $\operatorname{Imm}^{s o}(2,1)$, не имеет кривых сборки при проекции $\pi \circ \psi: N \rightarrow$ $\mathbb{R}^{2} \times I$ и имеет регулярную поверхность складки в смысле предшествуюшего определения. Определено естественное отображение $p: \operatorname{Imm}^{s o} \Sigma(2,1) \rightarrow \operatorname{Imm}^{s o}(2,1)$, которое согласно [19] является изоморфизмом.

Пусть $\psi: M^{2} \rightarrow \mathbb{R}^{3}$ - пленочная поверхность. Ориентируем поверхность $M^{2}$ так, что вектор внешней нормали к листу $M_{+}\left(M_{-}\right)$направлен в положительную (отрицательную) сторону оси проекции $\pi$. Заметим, что при таком выборе ориентации погружение $\psi$ обладает регулярной складкой.

Пусть $\varphi: M^{2} \rightarrow \mathbb{R}^{3}$ есть $C^{\infty}$-близкое к $\psi$ погружение общего положения. По особенностям $I$-структуры погружения $\varphi$ определим следующее числовое выражение:

$$
\bar{H}(\varphi)=\left(\delta_{++} ; \delta_{++}\right)+\left(\delta_{--} ; \delta_{--}\right)+\left(\delta_{+-} ; \delta_{+-}\right)+\left(\delta_{++}, \delta_{+-}\right)+i\left(q_{-}\right) \quad(\bmod 2) .
$$

Определим каждое слагаемое в этом выражении. Числа $\left(\delta_{++} ; \delta_{++}\right)$и $\left(\delta_{--} ; \delta_{--}\right)$были определены ранее (в частном случае пленочной поверхности) при формулировке предложения 5.1. Разобьем кривую $\pi \Delta$ на три компоненты $\pi \Delta_{++}, \pi \Delta_{--}, \pi \Delta_{+-}$в соответствии с обозначениями листов $M_{+}$и $M_{-}$поверхности $M$, образующих в пересечении рассматриваемую компоненту. 
Число $\left(\delta_{++} ; \delta_{++}\right)$определено как четность числа точек самопересечения кривой $\pi \Delta_{++}$на плоскости, которые возникают при проектировании. В частности, это означает, что точка тройного самопересечения поверхности не вносит вклада в выражение. Аналогично определим

$$
\begin{aligned}
& \left(\delta_{--} ; \delta_{--}\right)=\operatorname{ord}\left(\pi \Delta_{--} \odot \pi \Delta_{--}\right)(\bmod 2), \\
& \left(\delta_{+-} ; \delta_{+-}\right)=\operatorname{ord}\left(\pi \Delta_{+-} \odot \pi \Delta_{+-}\right)(\bmod 2) \text {, } \\
& \left(\delta_{++}, \delta_{+-}\right)=\operatorname{ord}\left(\pi \Delta_{++} \odot \pi \Delta_{+-}\right)(\bmod 2) \text {. }
\end{aligned}
$$

Определим разновидности $\pi Q_{+}$и $\pi Q_{-}$особенностей типа $\pi Q$. Скажем, что $q \in$ $\pi Q_{+}$, если один из листов поверхности, не содержаший кривую складки, имеет разновидность $M_{+}$. В противном случае $q \in \pi Q_{-}$. Для каждой точки $q_{j} \in \pi Q_{-}$определим $i\left(q_{-}\right)$как индекс точки $q_{j}^{\prime}$ относительно кривой $\pi \Delta$, где $q_{j}^{\prime}$ получена из $q_{j}$ в результате малого сдвига вдоль коориентирующего вектора к кривой $\pi \Sigma$. Далее определим $\operatorname{ind}\left(q_{-}\right)=\sum_{j} \operatorname{ind}\left(q_{j}\right)(\bmod 2), q_{j} \in \pi Q_{-}$

ЛЕмМа 6.1. Для поверхности ч с регулярной складкой выражение $\bar{H}(\varphi)$ не зависит от выбора погружения в классе кобордизма в группе $\operatorname{Imm} \Sigma^{\text {so }}(2,1)$.

ДокАЗАТЕльство. Пусть $\psi: N^{3} \rightarrow \mathbb{R}^{3} \times I-$ кобордизм погружений $\varphi_{1}$ и $\varphi_{2}$. Исследуем список изолированных особенностей $I$-структуры, малая окрестность которых содержит компоненты особых кривых $\pi \Delta \Delta, \pi Q$, но не содержит ветви кривой $\pi \Sigma^{1,1}$. Список состоит из следующих особенностей:

$$
\pi T, \quad \pi R, \quad \pi Q \odot \pi \Sigma^{1}, \quad \pi Q \odot \pi \Delta, \quad \pi \Delta \odot \pi \Delta \odot \pi \Delta, \quad \pi \Delta \odot \pi \Delta \odot \pi \Sigma^{1} .
$$

Докажем, что 1-коцикл $\bmod 2$, построенный по $\bar{H}$, когомологичен $0 \bmod 2$. Содержательная проверка возникает для случаев особенностей типов $\pi T, \pi R, \pi Q \odot \pi \Delta$.

Рассмотрим особенность $\pi Q \odot \pi \Delta$. Разберем два случая в зависимости от разновидности особой кривой $\pi Q_{+}$или $\pi Q_{-}$в окрестности особенности. К особенности, образованной $\pi Q_{+}$, примыкают две вертикальные ветви особой кривой $\pi \Delta \Delta$, которые имеют одну из трех возможных пар разновидностей:

$[(++),(++) ;(+-),(++)], \quad[(++),(--) ;(+-),(--)], \quad[(++),(+-) ;(+-),(+-)]$.

Напомним, что разновидности $(+-)$ и $(-+)$ совпадают. Значения коцепи $\bar{H}$ в каждом случае на паре вертикальных ветвей особой кривой совпадают. Две вертикальные ветви кривой $\pi Q_{+}$также имеют совпадающие значения коцепи $\bar{H}$.

Рассмотрим особенность $\pi Q \odot \pi \Delta$, в окрестности которой расположена кривая $\pi Q_{-}$. В окрестности этой особенности расположены две вертикальные ветви особой кривой $\pi \Delta \Delta$, которые имеют одну из трех возможных пар разновидностей:

$[(+-),(++) ;(--),(++)], \quad[(+-),(--) ;(--),(--)], \quad[(+-),(+-) ;(--),(+-)]$.

Значения коцепи $\bar{H}$ в каждом случае на паре вертикальных ветвей особой кривой различаются. В окрестности этой особенности расположено две ветви вертикальной кривой $\pi Q_{-}$, причем значения коцепи $\bar{H}$ на ветвях особой кривой различаются. 
Следовательно, в каждом разобранном случае коцепь $\bar{H}$ является коциклом в окрестности особой точки $\pi Q \odot \pi \Delta$.

Рассмотрим случай особенности $\pi T$. К особой точке примыкают пара вертикальных ребер особенности $\pi \Delta \Delta$ и две пары вертикальных ребер особенности $\pi Q$. Перечислим разновидности пары особых кривых типа $\pi Q$ :

$$
\left(\pi Q_{1,+}, \pi Q_{2,+}\right), \quad\left(\pi Q_{1,-}, \pi Q_{2,-}\right), \quad\left(\pi Q_{1,-}, \pi Q_{2,-}\right)
$$

Две пары вертикальных ребер $\pi \Delta \Delta$ имеют разновидности

$[(++),(+-) ;(+-),(++)], \quad[(+-),(+-) ;(--),(++)], \quad[(+-),(--) ;(--),(+-)]$.

В первом и третьем случаях значения коцикла $\bar{H}$ на соответствующих парах вертикальных ребер особенности $\pi \Delta \Delta$ совпадают. В первом случае значения $\bar{H}$ на каждой паре вертикальных ребер особенности $\pi Q$ совпадают, в третьем на каждой паре различаются. Во втором случае значение коцикла $\bar{H}$ на паре вертикальных ветвей кривой $\pi Q_{+}$совпадают, на паре ветвей кривой $\pi Q$ - различаются и на паре ветвей особой кривой $\pi \Delta \odot \pi \Delta$ различаются. Следовательно, $\bar{H}(\bmod 2)$ является коциклом в окрестности рассмотренных особенностей.

Рассмотрим случай особенности $\pi R$. К особой точке примыкают две пары вертикальных ребер особенности $\pi Q$ и одно ребро особенности $\pi \Delta \Delta$. Значение коцикла $\bar{H}$ на ребре $\pi \Delta \Delta$ равно 0 . Значения $\bar{H}$ на соответствуюших парах вертикальных ребер особенности $\pi Q$ совпадают. Мы доказали, что $\bar{H}$ является коциклом. Лемма 6.1 доказана.

Завершим доказательство формулы (15). Пусть $\psi: M^{2} \rightarrow \mathbb{R}^{3}$ - пленочная поверхность, $\varphi: M^{2} \rightarrow \mathbb{R}^{3}$ - погружение обшего положения, $C^{\infty}$-близкое к погружению $\psi$. При этом кривая складки $\Sigma$ ортогональной проекции поверхности на плоскость уже не лежит в этой выделенной плоскости.

Рассмотрим выражение $\bar{H}(\varphi) \bmod 2$. По лемме 6.1 это выражение не зависит от представителя в классе кобордизма. С другой стороны, очевидно равенство $H(\psi)=$ $\bar{H}(\varphi)$. Последнее утверждение легко проиллюстрировать примером. I-структура для поверхностей Константинова и $C^{\infty}$-близкой поверхности общего положения изображена на рис. 4 и 5 соответственно. По вычислениям предыдущего раздела получим $\bar{H}(\varphi)=H(\psi)=1(\bmod 2)$. Предложение доказано.

\section{7. Диаграмма Серфа двупараметрического семейства функций}

Пусть $C(M)$ - пространство псевдоизотопий гладкого односвязного многообразия $M, m=\operatorname{dim}(M)>5$. Для простоты изложения рассмотрим случай $M=S^{m}$, хотя все построения переносятся на случай произвольного ориентируемого многообразия. Зафиксируем на сфере $S^{m}$ стандартную риманову метрику и напомним определения, следуя [17]. Элемент пространства $C\left(S^{m}\right)$ представляется диффеоморфизмом $\alpha: S^{m} \times I \rightarrow S^{m} \times I$, причем $\left.\alpha\right|_{S^{m} \times\{0\}}=\mathrm{Id}$. Определено отображение $p: C=$ $C\left(S^{m}\right) \rightarrow H=H\left(S^{m}\right) \subset F=F\left(S^{m}\right)$, где $F-$ пространство функций $f: S^{m} \times I \rightarrow I$, $f\left(S^{m} \times i\right)=i, i=\{0\} ;\{1\}$, совпадающих со стандартной проекцией в малой окрестности границы, $H \subset F$ - подпространство функций, не имеющих критических точек. 
Отображение $p$ определено по формуле $\alpha \rightarrow \pi \circ \alpha$, где $\pi: S^{m} \times I \rightarrow I$ - стандартная проекция. Отображение $p$ является гомотопической эквивалентностью, и пространство $F$ является стягиваемым. Таким образом, при всех $s$ имеем $\pi_{s}(C)=\pi_{s}(H)=$ $\pi_{s+1}(F, H)$. Элемент этой гомотопической группы в интересующем нас случае $s=1$ может быть описан следующим образом.

Обозначим через $I(x), I(y), I(t), I(\tau)$ четыре экземпляра пространства единичного отрезка [0;1] с соответствуюшей координатой. Пусть $\Lambda: S^{m} \times I(x) \rightarrow I(y)$ - двупараметрическое семейство функций общего положения, $(t, \tau) \in I(t) \times I(\tau)$ такое, что ограничение семейства $\Lambda$ на границу $\partial(I(t) \times I(\tau))$ значений параметров не имеет критических значений. Ограничение семейства $\Lambda$ в точке $(t, \tau)$ пространства параметров будем обозначать через $\lambda(t, \tau)$. Внутри пространства значений+параметров множество критических значений семейства $\Lambda$ образует поверхность $S \rightarrow I(y) \times I(t) \times I(\tau)$. Гомотопиям представителей в заданном классе эквивалентности $\pi_{2}(F, H)$ соответствуют деформации семейства $\Lambda$ в классе описанных семейств в пространстве 3 параметров. В частности, семейство $\Lambda$ представляет нулевой элемент в относительной гомотопической группе тогда и только тогда, когда сушествует деформация $\Lambda \rightarrow \Lambda^{\prime}$, при которой деформация на границе пространства параметров $\partial(I(t) \times I(\tau))$ происходит в классе функций без критических значений, причем семейство $\Lambda^{\prime}$ также не имеет критических значений.

Особенности функций семейства $\Lambda$ описьваются локальньми нормальными формами, которые в интересуюшем нас случае $s=1$ имеют следуюший вид.

1. Невырожденная критическая точка (особенность типа Морса)

$$
-x_{1}^{2}-\ldots-x_{i}^{2}+x_{i+1}^{2}+\cdots+x_{m}^{2}+x_{m+1}^{2} .
$$

2. Критическая точка типа рождения или гибели пары морсовских критических точек при деформации в однопараметрическом семействе функций

$$
-x_{1}^{2}-\ldots-x_{i}^{2}+x_{i+1}^{2}+\cdots+x_{m}^{2}+x_{m+1}^{3} .
$$

3. Критическая точка типа "ласточкин хвост", образованная в результате слияния или распада пары особых точек типа "рождения или гибели" при деформации в двупараметрическом семействе функций

$$
-x_{1}^{2}-\ldots-x_{i}^{2}+x_{i+1}^{2}+\cdots+x_{n}^{2} \pm x_{n+1}^{4} .
$$

При двупараметрических деформациях функций общего положения критических точек более высокой кратности вырождения не встречается.

Согласно результатам [37] произвольное семейство $\Lambda$, определяющее элемент гомотопической группы $\pi_{2}(F, H)$, может быть продеформировано в семейство, имеюшее лиш особые точки типа 1 двух соседних индексов $i, i+1$ и точки типа 2 .

ОПРЕДЕЛЕНИЕ 7.1. Семейство функций $\Lambda$ называется $(i+1, i)$-нормальным (при этом подразумевается, что каждая функция $\lambda(t, \tau)$ семейства лежит в пространстве $\left.P^{S O}\left(S^{m}\right)\right)$, если выполнены два следующих условия.

1. Семейство $\Lambda$ не содержит особых точек типа “ласточкин хвост" и содержит только морсовские особые точки сигнатуры $i+1, i$, где $m>i+1>i>1$.

2. На каждом сепаратрисном диске семейства, восстановленном в критической точке семейства индекса $i+1$ или $i$, введена ориентация, непрерывно зависящая от выбора сепаратрисного диска в семействе. 
ЗАмЕчАниЕ. Правильное обобщение настоящего определения на случай семейств с большим числом параметров, содержаших высшие особенности, вероятно, связано с результатами В. А. Васильева [84], который построил комплекс особенностей вещественнозначных функций с учетом естественной коориентации стратов особенностей. Наш результат связан с рассмотрением простейших стратов $\Sigma^{1}=A_{1} \supset \Sigma^{1,1}=A_{2}$, при этом естественная коориентация на страте $\Sigma^{1}$ не определена, но вводится дополнительно условием 2 из определения 7.1. Важные результаты при изучении комплекса Васильева получены М.Э. Казаряном в работе [43].

Перейдем к формулировке основной теоремы. Для любого нормального (точнее, $(i, i+1)$-нормального) семейства определена поверхность критических значений $S$ и кривая $\Sigma \subset S$ критических значений типа “рождения-гибели”. При этом на кривой $\Sigma$ имеется дополнительная структура, так что определено разбиение на компоненты $\Sigma=\Sigma_{+} \cup \Sigma_{-}$, вообще говоря, несвязные. $\mathrm{K}$ компоненте $\Sigma_{+}$отнесем те критические точки, в окрестности которых рождается (погибает) пара морсовских особых точек индексов $(i+1, i)$, для которых коэффициент инцидентности сепаратрисных траекторий равен +1 . Если этот коэффищиент равен -1 , то соответствующая компонента кривой обозначается $\Sigma_{-}$. Определены групшы кобордизмов $\operatorname{Imm} L M(s), s=1,2$, $s$-параметрических нормальных семейств, причем отношение кобордантности задается $(s+1)$-параметрическими семействами, также удовлетворяющими условиям 1,2 . Определены гомоморфизмы

$$
\begin{aligned}
& \varkappa_{s}: \operatorname{ImmLM}(s) \longrightarrow \operatorname{Imm}^{s o}(s, 1), \\
& \rho_{s}: \operatorname{ImmLM}(s) \longrightarrow \operatorname{Imm}^{s o}(s-1,1) .
\end{aligned}
$$

Гомоморфизм $\varkappa_{2}$ сопоставляет двупараметрическому нормальному семейству $\Lambda$ класс кобордизма сглаженной погруженной поверхности $S^{\prime}$ критических значений в трехмерном пространстве $I(t) \times I(\tau) \times I(y)$ значений и параметров. При этом ориентация на поверхности критических значений нормального семейства выбирается такой, что вектор внешней нормали к листу поверхности, образованному критическими значениями индекса $i+1$, направлен в положительном направлении вдоль оси значений $I(y)$.

Гомоморфизм $\rho_{2}$ сопоставляет двупараметрическому нормальному семейству $\Lambda$ класс кобордизма кривой $\Sigma_{-}$критических значений типа "рождения-гибели" отрищательной разновидности (тот же класс кобордизма определен кривой $\Sigma_{+}$ положительной разновидности), которая проектируется на пространство параметров $I(t) \times I(\tau)$.

Теорема 3. Следующая диаграмма

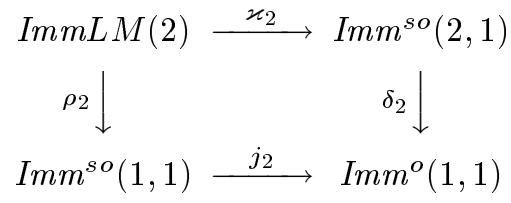

(в которой гомоморфизм $\delta_{2}$ определен в первой части работы как обратный $\kappa$ гомоморфизму Кана-Придди, изоморфизм $j_{2}$ индуцирован забыванием ориентации) оказывается коммутативной, причем оба гомоморфизма и2 и $\rho_{2}$ являются эпиморфизмами. 
(Для удобства читателя напомним, что в случае рассматриваемых размерностей оба гомоморфизма $\delta_{2}$ и $j_{2}$ являются изоморфизмами.)

ЗАмЕчАниЕ. В случае $s=1$ также определена коммутативная диаграмма

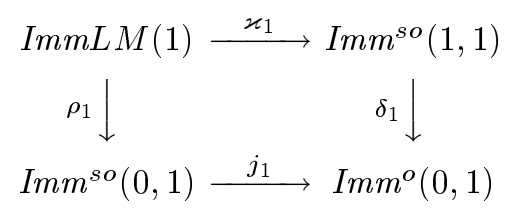

посредством аналогичных построений. Автору не известно, обобщается ли теорема 3 на случай семейств с числом параметров $s>2$ в предложенной форме. В работе [32] имеются обсуждения, относящиеся, возможно, к проблеме построения диаграммы при $s=3$.

ЗАмечАниЕ. Теорема 3 допускает эквивалентную переформулировку. Пусть $\varkappa_{i} \in \Pi_{i}, i=1,2,-$ элемент стабильной гомотопической групшы сфер, определенньй диаграммой Серфа $i$-параметрического семейства, $\rho_{i} \in \Pi_{i-1}$ - элемент стабильной гомотопической группы сфер, представленньй $(i-1)$-мерным подмногообразием особенностей сборки отрицательной разновидности. Справедливы тождества: $\alpha \circ \rho_{i}=\varkappa_{i}$, где $\alpha \in \Pi_{1}-$ образующая, $\Pi_{1} \circ \Pi_{i-1} \rightarrow \Pi_{i}-$ композиция Тоды в стабильных гомотопических группах сфер (см. введение).

ЗАмЕчАНИЕ. Ниже в предложении 8.3 приводится пример двупараметрического нормального семейства $\Lambda(t, \tau)$ такого, что погруженная поверхность $\varkappa_{2}(\Lambda)$ является образуюшей в группе кобордизмов $\operatorname{Imm}^{s o}(2,1)$. Этот пример строится по аналогии с примером образующей группы $\pi_{2}\left(F_{3}\left(\mathbb{R}^{1}\right)\right.$ ) (по поводу определений см. введение к ч. 2 ) из работы В. А. Васильева [86], см. также книгу [85; рис. 20]. Формально говоря, этот пример потребуется для доказательства эпиморфности гомоморфизма $\varkappa_{2}$. Эпиморфность гомоморфизма $\rho_{2}$ вытекает тогда из коммутативности диаграммы.

С ЛЕДСТВИЕ 7.2. Пусть поверхность $S$, моделирующая диаграмму Серфа двупараметрического нормального семейства функций, удовлетворяет условиям 1 и 2, сформулированным во введении, т.е. после сглаживания углов погруженная поверхность $S^{\prime}$ в пространстве $I(y) \times I(t) \times I(\tau)$ имеет ненулевой Arf-инвариант и, кроме того, проекиия каждой компоненты кривой критических значений типа "рождения-гибели" на плоскость $I(t) \times I(\tau)$ самопересекается в четном числе точек. Тогда поверхность $S$ не мохет быть реализована как поверхность критических значений диаграммы Серфа.

ДокАЗАТЕЛЬСТво. Пусть нашлось семейство $\Lambda$, для которого $S$ служит поверхностью критических значений. По условию $\varkappa_{2}(\Lambda)=1, \rho_{2}(\Lambda)=0$. По теореме 1.2 ч. 1 гомоморфизм $\delta: \operatorname{Imm}^{s o}(2,1) \rightarrow \operatorname{Imm}^{o}(1,1)$ является изоморфизмом. Эти условия противоречат коммутативности диаграммы (17). Следствие 7.2 доказано.

СлеДСТВИЕ 7.3. Пусть поверхность $S$ моделирует диаграмму Серфа канонического семейства функиий (определение см. в следующем разделе), при этом после сглаживания углов поверхность $S$ превращается в погруженную поверхность Константинова. Тогда поверхность $S$ не может служить диаграммой Серфа ни для какого канонического двупараметрического семейства функиий $\Lambda$. 
ЗАмЕчАниЕ. Если вдобавок потребовать выполнение нижеперечисленњых условий $1,2,3$, то предыдушее следствие вытекает из результатов Игусы [40].

1. Ограничение семейства $\Lambda$ на границу пространства параметров совпадает со стандартной проекцией на сомножитель $I(x)$.

2. Все компоненты кривой сборки имеют положительньй тип.

3. Все элементы из группы $\pi_{1}(S O(i+1))$, которые задают функцию склейки стабильных сепаратрисных дисков вдоль каждой компоненты кривой сборки, обрашаются в нуль.

ДОКАЗАТЕЛЬСТВО СЛЕДСТВИЯ 7.3. В соответствии с вычислениями раздела 5 Arf-инвариант поверхности Константинова равен 1, и, следовательно, поверхность удовлетворяет условиям в следствии 7.2. Доказательство закончено.

\section{8. Доказательство основной теоремы 3}

Изложим предварительные алгебраические сведения, следуя книге [57]. Пусть $L-$ кольцо (для простоты изложения коммутативное). Рассмотрим подгруппу $E(n, L) \subset$ $G L(n, L)$ в группе обратимых матрищ размера $n \times n$, порожденную всевозможными элементарными матрицами $e_{i, j}^{\lambda}$, где элемент $\lambda \in L$ стоит на $(i, j)$-месте, $1 \leqslant i, j \leqslant n$, $i \neq j$, все диагональные элементы равны 1 , а на остальных местах стоят нули. Определим $E(L)=\lim _{n} E(n, L)$ и $K_{2}(L)=H_{2}(E(L) ; \mathbb{Z})$. Группа $K_{2}(L)$ допускает описание в терминах образуюших и соотношений.

Пусть $n \geqslant 3$. Рассмотрим групу $S t(n ; L)$, порожденную образующими $x_{i, j}^{\lambda}$ и соотношениями

$$
\begin{gathered}
x_{i, j}^{\lambda} x_{i, j}^{\mu}=x_{i, j}^{\lambda+\mu}, \\
{\left[x_{j, i}^{\lambda}, x_{l, j}^{\mu}\right]=x_{l, i}^{-\lambda \mu}, \quad i \neq l, \quad \text { где }[a, b]=a b a^{-1} b^{-1},} \\
{\left[x_{i, j}^{\lambda}, x_{k, l}^{\mu}\right]=1, \quad j \neq k, \quad i \neq l .}
\end{gathered}
$$

Далее определим $S t(L)=\lim _{n} S t(n, L)$. Определен канонический гомоморфизм $\varphi: S t(L) \rightarrow G L(L)$ формулой $\varphi\left(x_{i, j}^{\lambda}\right)=e_{i, j}^{\lambda}$. Группа $K_{2}(L)$ совпадает с подгруппой $\operatorname{ker} \varphi$ и является центром группы $S t(L)$.

Нас будет интересовать случай, когда кольцо $L$ совпадает с кольцом целых чисел. Согласно теореме Милнора справедливо равенство $K_{2}(\mathbb{Z})=\mathbb{Z} / 2$, причем изоморфизм $t: K_{2}(\mathbb{Z}) \rightarrow \pi_{1}(S O)=\mathbb{Z} / 2$ устанавливается следуюшим правилом. Пусть $\prod_{s=1}^{k} x_{i_{s}, j_{s}}^{\lambda_{s}}$ представляет элемент из $K_{2}(\mathbb{Z})$. Определим отображение $h: S^{1} \rightarrow G L(\mathbb{R})$ по формуле $h(t)=\prod_{s=1}^{k} \varphi\left(x_{i_{s}, j_{s}}^{\lambda_{s} t}\right), 0 \leqslant t \leqslant 1$, где матрица $h(t)$, зависящая от параметра, представляется произведением обратимых унимодулярных матрищ над полем вешественных чисел. По построению $h(0)=h(1)=E$. Гомотопическая эквивалентность $S O \subset G L(\mathbb{R})$ определяет элемент $\pi_{1}(S O)=\mathbb{Z} / 2$ (см., например, [23], [66]), и гомоморфизм $t$ является изоморфизмом.

Нам потребуется несколько технических обозначений. Для числа $u \in \mathbb{Z}$ определим символ $w_{i, j}(-1)=x_{i, j}^{-1} x_{j, i}^{1} x_{i, j}^{-1}, h_{i, j}(-1)=w_{i, j}^{2}(-1)$. Матрица $\varphi\left(h_{i, j}(-1)\right)$ является диагональной, причем отличается от единичной только элементами $a_{i, i}=a_{j, j}=-1$. Определим $p_{i, j, k}=w_{i, j}(-1) h_{k, j}(-1)$, где все числа $i, j, k$ попарно различны. Матрица $\varphi\left(p_{i, j, k}\right)$ переставляет элементы базиса с номерами $(i, j)$ и обрашает вектор базиса 
с номером $k$. Далее для сокрашения формул будем использовать обозначение $p_{i, j, k}$ (и аналогичные обозначения) не только для символа, но и для матрицы.

Напомним основные конструкции работы [33], в которой соответствуюшие построения проводились для более общего случая неодносвязного многообразия. Пусть $\lambda: S^{m} \times I \rightarrow I-$ функция Морса, имеющая лишь невырожденные критические точки индексов $i+1, i$ с попарно различными критическими значениями. Предположим, что в окрестности каждой критической точки индекса $i+1$ зафиксирована ориентация стабильного сепаратрисного диска размерности $i+1$ и в окрестности критической точки индекса $i$ зафиксирована ориентация нестабильного сепаратрисного диска размерности $m+1-i$. Упорядочим множества $\left\{a_{1}, \ldots, a_{q}\right\},\left\{b_{1}, \ldots, b_{q}\right\}$ критических точек индексов $i+1, i$ в соответствии с возрастанием (убыванием) критических значений. Определен цепной комплекс $\mathbb{Z}$-модулей

$$
0 \longrightarrow C_{i+1} \stackrel{A}{\longrightarrow} C_{i} \longrightarrow 0
$$

порожденный клеточным разбиением многообразия $S^{m} \times I(x)$, построенным по функции $\lambda$ и ориентации сепаратрисных дисков. Комплекс (21) точен, и группы цепей снабжены выделенными базисами, согласованными с нумерацией критических точек.

Пусть $\lambda(t): S^{m} \times I \rightarrow I$ - однопараметрическое семейство функций общего положения, $t \in I(t)$. Для всех значений параметра $t$, кроме конечного числа особых значений, функция $\lambda(t)$ является морсовской с различными критическими значениями. На многообразии $S^{m} \times I$ зафиксируем стандартную риманову метрику. Для конечного числа значений параметра $t$ функция $\lambda(t)$ имеет особенности одного из следующих типов.

А1. Особенность типа "рождения или гибели" пары морсовских критических точек. В соответствии со значениями коэффициентов инщидентности сепаратрисных дисков различаются две разновидности особенности $\Sigma_{+}, \Sigma_{-}$. Условимся, что в окрестности каждой такой особенности стабильньй $(i+1)$-мерный и нестабильньй $(m-i+1)$ мерньй сепаратрисные диски двух морсовских особых точек не содержат никаких сепаратрисных траекторий, за исключением единственной, которая сами точки соединяет. Этого можно добиться в результате дополнительной деформации функции (не являющейся, вообще говоря, деформацией общего положения) в малой окрестности критической точки, после которой все дополнительные траектории сокращаются при помощи особенностей А2, А4 (см. ниже).

А2. Особенности $V_{+}\left(V_{-}\right)$образованы градиентными траекториями, соединяющими два критических морсовских значения одного индекса $i+1$ ( $i$ соответственно). При этом для сокращения формулировок под особенностью указанного типа мы иногда будем понимать концевые точки особой траектории, что позволит нам рассматривать эти особенности не только в многообразии $S^{m} \times I(x)$, но и в пространстве параметров+значений. Для удобства читателя опишем эту особенность подробнее.

Пусть при $t=t_{0}-\varepsilon$ функция $\lambda(t)$ имеет две критических точки $a, b$ индекса $i$ с различными критическими значениями $\lambda(a)<y_{0}<\lambda(b)$. Пусть $D_{+}(a)$ - сепаратрисньй диск, образованньй входящими градиенто-подобными траекториями функции $\lambda(t)$ в критической точке $a$. Этот диск имеет размерность $n+1-i$ и пересекает подмногообразие $\lambda^{-1}\left(y_{0}\right) \subset S^{n} \times I$ по вложенной сфере $S_{a}^{n-i}(t)$ размерности $n-i$. Аналогично, пусть $D_{-}(b)$ - сепаратрисный диск размерности $i$, образованный выходящими градиенто-подобными траекториями функции $\lambda(t)$ в критической точке $b$. Этот диск 
пересекает то же многообразие $\lambda^{-1}\left(y_{0}\right)$ по вложенной сфере $S_{b}^{i-1}(t)$. Особенность $V_{-}$ связана с точкой трансверсального пересечения сфер $S_{a}^{n-i}(t), S_{b}^{i-1}(t)$ в многообразии $\lambda^{-1}\left(y_{0}\right)$, образованной в момент времени $t=t_{0}$. Особая сепаратрисная траектория, соединяющая критические точки $a, b$ индекса $i$, проходит через точку пересечения $S_{a}^{n-i}\left(t_{0}\right)$ и $S_{b}^{i-1}\left(t_{0}\right)$. Описание особенности $V_{+}$аналогично.

А3. Особенность $\Delta_{+}\left(\Delta_{-}\right)$совпадения двух критических значений одинаковых индексов $i+1$ ( $i$ соответственно). Особенность $\Delta_{+,-}$совпадения двух критических значений разных индексов.

A4. Особенность сокращения пары сепаратрисных траекторий с противоположными алгебраическими знаками, соединяющих пару критических точек соседних индексов.

Рассмотрим особенности двупараметрического нормального семейства общего положения $\Lambda(t, \tau)$, представляюшего элемент $\operatorname{Im} m L M(2)$. Поверхность критических значений такого семейства имеет особенности типов А1-A4 и изолированные особые точки следуюших четырех типов.

В1. Изолированные особенности $I$-структуры поверхности критических значений в пространстве значений-параметров.

В2. Особенности пересечения обшего положения особых кривых $V_{+}, V_{-}$с особенностями $I$-структуры поверхности критических значений.

В3. Изолированные особые сепаратрисные траектории $W_{+}$и $W_{-}$, связанные с соотношениями $(19),(20)$ в групше $S t(\mathbb{Z})$. Концевые точки этих траекторий служат вершинами одномерного графа с ребрами, образованными концевыми точками особых траекторий $V_{+}, V_{-}$.

В4. Особенности типа “обмена” сепаратрисных траекторий. Эти особенности связаны с перестройками особых траекторий, соединяющих критические точки одинакового индекса. (Определения и свойства см. в [37].) Каждая особенность этого вида является отрезком сепаратрисы в $S^{m} \times I(x)$, конщы которого соединяют вершины одномерного графа особых кривых $V_{+}, V_{-}$. Эту особенность можно рассматривать не только в многообразии $S^{m} \times I(x)$, но и на диаграмме Серфа. Для удобства читателя опишем эту особенность подробнее.

Пусть при значениях параметров $t=t_{0}, \tau=\tau_{0}$ функция $\Lambda(t, \tau)$ имеет критическую точку $a$ индекса $i$ и критическую точку $b$ индекса $i+1$, причем $\Lambda(a)>y_{0}>\Lambda(b)$, так что функция $\Lambda(t, \tau)$ в окрестности рассматриваемых значений параметров не является правильной. Пусть $D_{-}(a)$ - сепаратрисный диск точки $a$, образованньй выходящими сепаратрисными градиенто-подобньми траекториями размерности $i$. Пусть $D_{+}(b)-$ сепаратрисный диск точки $b$ размерности $n-i$, образованный входящими градиенто-подобными траекториями размерности $n-i$. Диски $D_{-}(a)$ и $D_{+}(b)$ пересекают подмногообразие уровня $\lambda^{-1}\left(y_{0}\right)$ по сферам $S_{a}^{i-1}(t, \tau), S_{b}^{n-i-1}(t, \tau)$ размерности $i-1$, $n-i-1$ соответственно. При $(t, \tau)=\left(t_{0}, \tau_{0}\right)$ сферы $S_{a}^{i-1}\left(t_{0}, \tau_{0}\right), S_{b}^{n-i-1}\left(t_{0}, \tau_{0}\right)$ имеют точку самопересечения, неустранимую при рассмотрении деформации особенности в двупараметрическом семействе. Отрезок особой сепаратрисной траектории проходит через эту точку.

Для дальнейших рассмотрений нам потребуется случай специальных семейств не общего положения.

ОПРЕДЕЛЕНИЕ КАНОНИЧЕСКОГО ДВУПАРАМЕТРИЧЕСКОГО СЕМЕЙСТВА ФУНК- 
ций. Скажем, что семейство $\Lambda$ является каноническим, если вьполнено следующее условие: поверхность критических значений $S \hookrightarrow I_{y} \times I_{t} \times I_{\tau}$ является пленочной в смысле определения из раздела, при этом особые кривые типа "рождения-гибели", соответствуюшие точкам складки пленочной поверхности, имеют критические значения $1 / 2$.

Чтобы иметь возможность перестраивать особенности В4 типа “обмена" сепаратрисных траекторий, требуется, чтобы диаграмма Серфа канонического семейства допускала особенности $\pi \Sigma \Sigma$ двух различных разновидностей. Сформулируем это утверждение подробнее.

C1. Особенности $\pi \Sigma \Sigma$ допускают две разновидности. Особенности первой разновидности являются границами пары ветвей каждой из кривых $V_{+}$и $V_{-}$. Окрестность особенности $\pi \Sigma \Sigma$ второй разновидности не содержит кривых $V_{+}$и $V_{-}$.

Поясним, как устроены особенности обеих разновидностей. Пусть $\lambda\left(t, \tau_{0}-\varepsilon_{2}\right)$, $t \in\left[t_{0}-\varepsilon_{1} ; t_{0}+\varepsilon_{1}\right],-$ однопараметрическое семейство функций (по параметру $t$ ), заданное в малой окрестности критической точки $\left(t_{0} ; \tau_{0}\right)$ типа $\mathrm{C} 1$, где $1 \gg \varepsilon_{1} \gg$ $\varepsilon_{2}>0$. В окрестности критического значения расположены две ветви кривой диаграммы Серфа. Предположим, что при каждом значении параметра $\varepsilon_{2}$ семейство функций $\lambda$ имеет особую точку типа $\Delta_{+}$при $t=t_{1}\left(\varepsilon_{2}\right)$, особую точку типа $\Delta_{-}$при $t=t_{2}\left(\varepsilon_{2}\right)$ и две особых точки типа "гибели" при значениях $t$, превышаюших $t_{2}$, причем $t_{1}\left(\varepsilon_{2}\right)>t_{2}\left(\varepsilon_{2}\right)$ при каждом $\varepsilon_{2}>0$. При $\varepsilon_{2} \rightarrow 0$ имеем $t_{1}=t_{2}=t_{0}$. Пусть при каждом $\varepsilon_{2}$ при некотором значении $t_{3}\left(\varepsilon_{2}\right)$ параметра $t$ на паре ветвей диаграммы имеется особенность типа $V_{+}$и при некотором $t_{4}$ имеется особенность $V_{-}$, причем $t_{3}>t_{1}$. В зависимости от условий $t_{4}>t_{2}$ или $t_{4}<t_{2}$ следует различать два случая. В первом случае дополнительной деформацией особенности $V_{+}$и $V_{-}$удается сократить так, что в окрестности особого значения $\left(t_{0}, \tau_{0}\right)$ особенностей типов $V_{+}$и $V_{-}$не появляется, этот случай соответствует особенности $\mathrm{C} 1$ второй разновидности. Во втором случае сократить особенности, не меняя при этом структуру самопересечения поверхности критических значений, не удается. Этот случай соответствует особенности первой разновидности.

Фактически, рассмотрение канонических семейств не приводит к потере общности. Более формально, справедливо следуюшее предложение, которое доказано в [5].

ПРЕДЛОЖЕНИЕ 8.1. Произвольнъй класс кобордизма нормальньх семейств из группь $\operatorname{ImmLM(2)~представляется~каноническим~семейством.~}$

Конструкция 8.2. Опишем ключевую конструкцию, необходимую для доказательства основной теоремы. Пусть $\Lambda(t, \tau)$ - каноническое семейство функций. Пусть $\tau_{0}$ - неособое значение параметра деформации, при котором семейство $\lambda(t)=\left.\Lambda(t ; \tau)\right|_{\tau=\tau_{0}}$ имеет особенности типов A1-А3. Определим элемент $\Phi(\tau) \in K_{2}(\mathbb{Z})$.

Пусть $t=t_{0}-$ значение параметра деформации, при котором функция $\left.\lambda(t)\right|_{t=t_{0}}$ является правильной функцией Морса. Пусть $A\left(t_{0}\right): C_{k+1} \rightarrow C_{k}$ - оператор границы в цепном комплексе $(21), \operatorname{dim}\left(C_{k}\right)=\operatorname{dim}\left(C_{k+1}\right)=s$. Определим расширения групп цепей $\bar{C}_{k+1}, \bar{C}_{k}$, добавляя две образующие, индексированные 0 и -1 . Построим оператор $\bar{A}\left(t_{0}\right): \bar{C}_{k+1} \rightarrow \bar{C}_{k}$, который будем назьвать оператором в пополненном цепном 
комплексе. Зададим матрищу оператора $\bar{A}\left(t_{0}\right)$ по формуле:

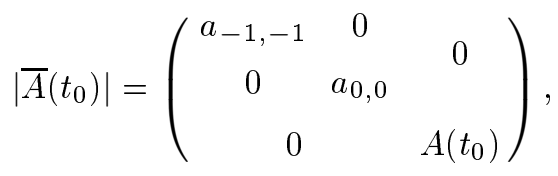

где числа $a_{-1,-1}, a_{0,0}$ принимают значения $+1,-1$ и вычисляются согласно следующему правилу. Пусть $a\left(t_{0}\right)$ - количество особенностей типа $\Sigma_{-}$семейства $\lambda(t)$ в промежутке значений параметра $0<t \leqslant t_{0}$. Пусть $b\left(t_{0}\right)-$ количество особенностей типа $\Delta$ совпадения пары критических значений при тех же значениях параметра. Заметим, что четность чисел $a\left(t_{0}\right)$ и $b\left(t_{0}\right)$ не изменится, если перейти к рассмотрению соответствующих особенностей на промежутке $t_{0}<t \leqslant 1$. Определим $a_{-1,-1}=(-1)^{b\left(t_{0}\right)}$, $a_{0,0}=(-1)^{a\left(t_{0}\right)}$.

Пусть $T=\left\{t_{1}, \ldots, t_{l}\right\}$ - множество особых значений параметра в семействе $\lambda(t)$. Опишем цепочку элементарных преобразований матриц расширенных операторов $\bar{A}(t)$ в окрестности каждого критического значения параметра из множества $T$ :

$$
\begin{aligned}
E=\bar{A}\left(t_{1}-\varepsilon\right) & \stackrel{w\left(t_{1}\right)}{\longrightarrow} \bar{A}\left(t_{1}+\varepsilon\right)=\bar{A}\left(t_{2}-\varepsilon\right) \stackrel{w\left(t_{2}\right)}{\longrightarrow} \cdots \\
& \ldots \stackrel{w\left(t_{l-1}\right)}{\longrightarrow} \bar{A}\left(t_{l-1}+\varepsilon\right)=\bar{A}\left(t_{l}-\varepsilon\right) \stackrel{w\left(t_{l}\right)}{\longrightarrow} \bar{A}\left(t_{l}+\varepsilon\right)=E
\end{aligned}
$$

Начальньй и конечньй члены цепочки образованы единичной матрицей $E$. Разберем случаи различных типов особенностей и определим преобразования $w$ для каждого типа в соответствии со следуюшими правилами.

1. Особенность типа $\Sigma$. Пусть функция $\lambda\left(t_{i}\right), t_{i} \in T$, имеет особую точку типа "рождения" пары морсовских критических точек $e_{s}, f_{s}$. В дальнейшем соответствуюший символ, например $h_{0, q}(-1)$, мы будем понимать так же, как и матрицу $\varphi\left(h_{0, q}(-1)\right)$, для сокрашения обозначений. Рассмотрим две разновидности особенности. Для особенности разновидности $\Sigma_{+}$, т.е. в случае $\operatorname{lk}\left(e_{q}, f_{q}\right)=+1$ (где $\mathrm{lk}-$ коэффициент инцидентности), определим $w\left(t_{i}\right)$ как тождественное преобразование. Для особенности разновидности $\Sigma_{-}$, т.е. в случае $\operatorname{lk}\left(e_{q}, f_{q}\right)=-1$, преобразование определим по формулам $\bar{A}\left(t_{i}+\varepsilon\right)=\bar{A}\left(t_{i}-\varepsilon\right) h_{0, q}(-1)$. Для особенности типа "гибели" пары морсовских критических точек определим $w\left(t_{i}\right)$ обратными формулами. В частности, для особенности разновидности $\Sigma_{-}$определим $\bar{A}\left(t_{i}+\varepsilon\right)=\bar{A}\left(t_{i}-\varepsilon\right) h_{0, q}^{-1}(-1)$.

2. Особенность типа $V$. Преобразование $w\left(t_{i}\right)$ определено нижеследуюшими формулами в соответствии с разновидностью особенности, подробнее см. [37].

$V_{+} \cdot \bar{A}\left(t_{i}+\varepsilon\right)=e_{j, k}^{\lambda} \bar{A}\left(t_{i}-\varepsilon\right)$.

$V_{-} . \bar{A}\left(t_{i}+\varepsilon\right)=\bar{A}\left(t_{i}-\varepsilon\right) e_{k, j}^{\lambda}$.

3. Особенность типа $\Delta$. Будем различать два типа преобразований в соответствии с разновидностями особенности $\Delta_{+}, \Delta_{-}$. Каждый тип преобразования подразделяется на две разновидности в соответствии со значением коэффициента $a_{-1,-1}$ в матрице $\bar{A}\left(t_{i}+\varepsilon\right)$ оператора.

$$
1_{(+)} \cdot \Delta_{+} \cdot m_{-1,-1}\left(t_{i}-\varepsilon\right)=1 \text {. }
$$

$$
\bar{A}\left(t_{i}+\varepsilon\right)=p_{j, j+1,-1} \bar{A}\left(t_{i}-\varepsilon\right) .
$$




$$
\begin{array}{r}
1_{(-)} \cdot \Delta_{+} \cdot m_{-1,-1}\left(t_{i}-\varepsilon\right)=-1 \\
\bar{A}\left(t_{i}+\varepsilon\right)=\left[p_{j, j+1,-1}\right]^{-1} \bar{A}\left(t_{i}-\varepsilon\right) . \\
2_{(+)} . \Delta_{-} . m_{-1,-1}\left(t_{i}-\varepsilon\right)=1 . \\
\bar{A}\left(t_{i}+\varepsilon\right)=\bar{A}\left(t_{i}-\varepsilon\right) p_{j, j+1,-1} . \\
2_{(-)} \cdot \Delta_{-} . m_{-1,-1}\left(t_{i}-\varepsilon\right)=-1 . \\
\left.\bar{A}\left(t_{i}+\varepsilon\right)=\overline{(} t_{i}-\varepsilon\right)\left[p_{j, j+1,-1}\right]^{-1} .
\end{array}
$$

Описанная цепочка преобразований (23) определяет элемент $\Phi(\tau) \in S t(\mathbb{Z})$. По построению $\varphi(\Phi(\tau))$ совпадает с единичной матрицей, в частности, $a_{-1,-1}=a_{0,0}=1$. Таким образом, $\Phi(\tau) \in K_{2}(\mathbb{Z})$. Описание конструкции завершено.

ДокАЗАТЕЛЬСтво ТЕоРЕмы 3. Изложим краткое доказательство, а полное доказательство можно найти в [8]. Согласно предложению 8.1 достаточно доказать коммутативность диаграммы (17) для случая канонического семейства $\Lambda(t, \tau)$. Рассмотрим функцию $\Phi(\tau)$, зависящую от переменной $\tau \in I(\tau)$. Изучим точки разрьва функции на всей области определения. Необходимо проверить, что функция $\Phi(t)$ непрерывна при всех значениях параметра $\tau$, за исключением критических значений с особенностями $\pi \Delta \Delta, \pi \Sigma \Sigma$.

В особых точках $\pi \Delta \Delta$ непрерывность функции зависит от разновидности особенности. В точках пересечения особых кривых $\pi \Delta_{+}$и $\pi \Delta_{-}$функция $\Phi(\tau)$ непрерьвна. В точках самопересечения особых кривых $\pi \Delta_{+}$и $\pi \Delta_{-}$функция имеет разрыв.

В особых точках $\tau$ типа $\pi \Sigma \Sigma$ функция изменяется в соответствии с формулой

$$
\Phi\left(\tau_{i}-\varepsilon\right)-\Phi\left(\tau_{i}+\varepsilon\right)=\frac{1}{2}\left[(-1)^{\left[\operatorname{sign}\left(x_{i}\right)+\operatorname{Ind}\left(x_{i}\right)\right]}-1\right] \quad(\bmod 2),
$$

где $\operatorname{sign}\left(x_{i}\right)=1$, если $x_{i} \in \pi \Sigma_{-} \odot \pi \Sigma_{-}$, и $\operatorname{sign}\left(x_{i}\right)=0$, если $x_{i} \in \pi \Sigma_{+} \odot \pi \Sigma_{+}$или $x_{i} \in \pi \Sigma_{+} \odot \pi \Sigma_{-}, \operatorname{Ind}\left(x_{i}\right)$ - индекс особой точки, определенньй в разделе 5 .

Доказательство теоремы 3 основьвается на формуле (24). Обозначим через $\operatorname{ord}[\delta(\Phi)]$ четность числа точек разрьва функции. Поскольку $\Phi(0)=\Phi(1)$, справедливо равенство ord $[\delta(\Phi)]=0$. С другой стороны, это число может быть вычислено по формуле

$$
\operatorname{ord}[\delta(\Phi)]=\sum_{i}(-1)^{\operatorname{sign}\left(x_{i}\right)+\operatorname{Ind}\left(x_{i}\right)}+\left(\delta_{++} ; \delta_{++}\right)+\left(\delta_{--} ; \delta_{--}\right) \quad(\bmod 2) .
$$

В соответствии с формулой (15) указанная сумма равна $\operatorname{Arf}(S)+\operatorname{ord}\left(\pi \Sigma \Sigma_{--}\right)$, где первое слагаемое обозначает класс кобордизма поверхности критических точек, за-

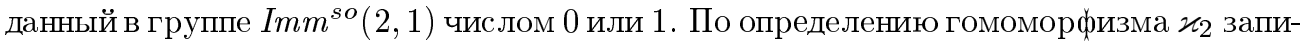
шем $\operatorname{Arf}(S)=\varkappa_{2}(\Lambda)$. Значение второго слагаемого определяется выражением $\rho_{2}(\Lambda)$ в группе кобордизма $\operatorname{Imm}^{s o}(1,1)$ и также определяется числом 0 или 1 . Запишем $\operatorname{ord}\left(\pi \Sigma \Sigma_{-,-}\right)=\rho_{2}(\Lambda)$. Коммутативность диаграммы $(17)$ доказана.

Для завершения доказательства теоремы 3 осталось воспользоваться следуюшим предложением, доказанным в [5].

ПРЕДЛОЖЕНИЕ 8.3. В диаграмме (17) отображение ћ2 является әпиморфизмом. 


\section{9. Аппроксимация вложениями отображений общего положения поверхностей в объемлющем пространстве $\mathbb{R}^{4}$. Теорема Рохлина о сигнатуре и аналоги инварианта странности для диаграммы узла}

Пусть $f: M^{2} \rightarrow \mathbb{R}^{3}$ - гладкое отображение общего положения замкнутой ориентированной поверхности. При каких условиях композиция $i \circ f: M^{2} \rightarrow \mathbb{R}^{3} \rightarrow \mathbb{R}^{4}$ аппроксимируется $\varepsilon$-близким гладким вложением $g: M^{2} \rightarrow \mathbb{R}^{4}$ для произвольного $\varepsilon>0$ ? Другими словами, когда отображение $f$ дискретно реализуется в пространстве $\mathbb{R}^{4}$ ? Следуюшее предложение дает ответ на поставленный вопрос.

ПРЕДЛОЖЕНИЕ 9.1. Отображение $f$ дискретно реализуется в пространстве $\mathbb{R}^{4}$ тогда и только тогда, когда существует гладкое вложсние $\bar{f}: M^{2} \rightarrow \mathbb{R}^{4}$ такое, что $f=\pi \circ \bar{f}$, где $\pi: \mathbb{R}^{4} \rightarrow \mathbb{R}^{3}$ - стандартная проекция.

ЗАмЕчАниЕ. Предложение 9.1 сформулировано в виде гипотезы в работе [13] . Ниже в 9.6-9.9 приводится конструкция (частичного) алгебраического препятствия к решению задачи реализации. Эта конструкция получена в совместной работе автоpa [13] и обобшает алгебраическую конструкцию из [6].

Отложим доказательство предложения 9.1 и сформулируем вначале необходимые и достаточные условия сушествования вложения $\bar{f}$. Будем говорить, что отображение $f$ является Prem-отображением (Prem = projected embedding), см. [81], если вложение $\bar{f}$ с указанньми свойствами существует. Далее в этом разделе для сокрашения будем подразумевать под поверхностью не только $M^{2}$, но и образ отображения $f$ общего положения.

Напомним структуру особенностей для поверхности обшего положения в трехмерном пространстве. Кривая $\Delta_{2}$ точек самопересечения поверхности состоит из конечного числа замкнутых компонент и некоторого числа компонент с краем, погруженных в пространство. Концевые точки незамкнутых компонент соответствуют особым точкам отображения $f$, имеюшим тип зонтика Уитни. Кривая $\Delta_{2}$, которую далее для краткости будем обозначать через $\Delta$, вообше говоря, самопересекается в конечном множестве особых точек с кратностью 3 . Эти точки самопересечения будут обозначаться через $\Delta_{3}$, они соответствуют точкам тройного самопересечения поверхности в пространстве.

ОПРЕДЕЛЕнИЕ Prem-СтРУКтуРЫ. Локальной Prem-структурой на поверхнос$m u f\left(M^{2}\right)$ назьвается ориентация $O=\left\{O_{i}\right\}$ или, точнее, набор ориентаций, заданных на каждой связной компоненте $\Delta(i)$ кривой двойных точек.

Заметим, что кривая $f^{-1}(\Delta)=\bar{\Delta}$ является погруженным многообразием в $M^{2}$ и определено двулистное накрытие $p: \bar{\Delta} \rightarrow \Delta$ всюду, за исключением граничных точек кривой $\Delta$. По предположению $M^{2}$ ориентирована и, следовательно, накрытие $p$ является тривиальным. Ориентация $O$ позволяет определить каноническое разбиение

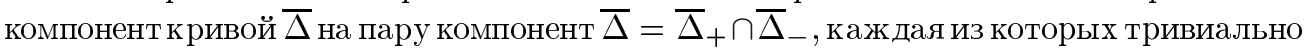
накрывает кривую $\Delta$. K компоненте $\bar{\Delta}_{+}$отнесем те ветви кривой, для которых упорядоченный базис пространства $\mathbb{R}^{3}$, составленный из векторов $\left(O(x), \xi_{+}(x), \xi_{-}(x)\right)$, правоориентирован, где $x$ точка на кривой $\Delta ; \xi_{+}(x)\left(\xi_{-}(x)\right)$ - вектор нормали к ориентированному листу поверхности, содержашему ветвь кривой $\bar{\Delta}_{+}\left(\bar{\Delta}_{-}\right.$соответственно), приложенный в точке $x$. 
Обозначим через $L_{i}, i=1,2,3$, листы поверхности $f\left(M^{2}\right)$ в малой окрестности трехкратной точки самопересечения $y \in \Delta_{3}$, упорядоченные произвольным образом. Пусть $o_{i}$ - векторы ориентации к кривой $\Delta$, приложенные в точке $y$, при этом $o_{i}$ трансверсально $L_{i}$. Точке $y$ сопоставим неупорядоченную тройку чисел, составленную из +1 или -1 . Припишем вектору $o_{i}$ значение +1 , если этот вектор сонаправлен с вектором нормали к ориентированному листу поверхности $L_{i}$, в противном случае припишем вектору значение -1 .

ОПРЕДЕЛЕНИЕ 9.2. Скажем, что точка $y \in \Delta_{3}$ uмеет mun $\mathrm{A}$, если ей приписана тройка чисел $(+1 ;+1 ;-1)$ (разновидность $\left.A_{+}\right)$или тройка чисел $(+1 ;-1 ;-1)$ (разновидность $A_{-}$). Скажем, что точка $y$ имеет mun $\mathrm{B}$, если ей приписана тройка $(+1 ;+1 ;+1)$ (разновидность $\left.B_{+}\right)$или тройка $(-1 ;-1 ;-1)$ (разновидность $\left.B_{-}\right)$.

КРИТЕРИЙ 9.3. Гладкое отображение $f$ общего положения тогда и только тогда является Prem-отображсением, когда существует ориентация $О$ на кривой $\Delta$, задающая локальную Prem-структуру, для которой все точки множества $\Delta_{3}$ имеют тип $\mathrm{A}$.

ЗАмечАниЕ. Критерий был ранее сформулирован в других терминах в работе [29]. Использование понятия локальной Prem-структуры на кривой $\Delta$, о котором сообшил автору А. Сюч, позволило сушественно упростить формулировку.

ДОКАЗАТЕЛЬСТВО КРИТЕРИЯ изЛожено в [6].

СлЕДСТВИЕ 9.4. Существует погружение $\varphi: S^{2} \rightarrow \mathbb{R}^{3}$, которое дискретно не реализуется в $\mathbb{R}^{4}$.

ДокАЗАТЕЛЬСтво. В качестве погружения $\varphi$ можно выбрать двулистное накрытие $S^{2} \rightarrow \mathbb{R P}^{2} \rightarrow \mathbb{R}^{3}$ над поверхностью Боя $\varkappa: \mathbb{R P}^{2} \rightarrow \mathbb{R}^{3}$, приведенное малой деформацией в общее положение в пространстве $\mathbb{R}^{3}$ как граница погруженной регулярной трубчатой окрестности. Подробности см. [6].

ОСНОВНОЕ СЛЕДСТВИЕ ПРЕДЛОЖЕНИЯ 9.1. Пространство $C^{0}$-отображений $\left[S^{2} ; \mathbb{R}^{3}\right]$, снабженное компактно-открытой топологией, содержит открытое и всюду плотное подпространство $\Re$, состоящее из отображсний, которые дискретно не реализуются в $\mathbb{R}^{4}$.

ДокАЗАТЕльство. Пусть $\widehat{\Re} \subset\left[S^{2} ; \mathbb{R}^{3}\right]$ - дополнение к указанному подпространству, которое описьвается как подпространство тех отображений $f: S^{2} \rightarrow \mathbb{R}^{3}$, для которых при любом $\varepsilon>0$ сушествует вложение $g: S^{2} \subset \mathbb{R}^{4}$ такое, что $\|I \circ f-g\|<\varepsilon$, $I: \mathbb{R}^{3} \rightarrow \mathbb{R}^{4}$. По определению $\bar{\Re}$ замкнуто. Действительно, если $f_{0}$ лежит в замыкании $\bar{\Re}$, то найдется последовательность $g_{i}: S^{2} \subset \mathbb{R}^{4}$ вложений, сходящихся к $f_{0}$, что доказывает $f_{0} \in \bar{\Re}$.

Пространство $\Re$ является всюду плотным. Действительно, для произвольного $f \in$ $\left[S^{2} ; \mathbb{R}^{3}\right]$ можно определить отображение $g=f \# \varkappa$, где $\varkappa$ построено в следствии 9.4. Выбирая $\operatorname{diam}(\varkappa(\varepsilon))$ достаточно малым, получим $\|f-g\|<\varepsilon$. Следствие доказано.

ЗАмЕчАниЕ. Связь предыдушего следствия с общей проблематикой вложимости полиэдров и аппроксимаций вложениями отображений полиэдров в евклидовы пространства исследуется в работах [13], [70]. 
Оказывается, с выбором произвольной локальной Рrem-структуры поверхности $f\left(M^{2}\right)$ связана квадратичная форма $q: H=H_{1}\left(M^{2} ; \mathbb{Z} / 2\right) \rightarrow \mathbb{Z} / 2$, позволяющая вычислить четность числа тройных точек типа В и препятствующая нахождению вложения $\bar{f}$, которое разрешает в 4 -пространстве особую кривую $\Delta$ в соответствии с заданной локальной Prem-структурой.

Определим квадратичную форму $q$ в соответствии со следуюшим правилом. Пусть $l: S^{1} \subset M^{2}, L=l\left(S^{1}\right)$ - вложенная петля, определяющая цикл $x \in H_{1}\left(M^{2} ; \mathbb{Z} / 2\right)$. Обозначим через $\operatorname{ord}\left(L \cap \bar{\Delta}_{+}\right)$четность числа точек пересечения кривых $L$ и $\bar{\Delta}_{+}$на поверхности $M$. Обозначим через $\operatorname{lk}(L, \psi)$ четность коэффициента самозацепления оснашенной кривой $(L, \psi)$ в пространстве, причем оснашение $\psi$ образовано векторами положительной нормали поверхности, восстановленными в точках кривой $L$.

ОпРЕДЕЛЕНИЕ 9.5. Определим значение квадратичной формы по формуле

$$
q(f, O)(x)=\operatorname{lk}(L, \psi)+\operatorname{ord}\left(L \cap \bar{\Delta}_{+}\right)
$$

Лемма 9.6. Значение $q(f, O)(\bmod 2)$ не зависит от выбора иикла в классе гомологий, и функиия q является квадратичной формой, ассочиированной с билинейной формой $\langle H, H\rangle$, определенной операцией пересечения неориентированных ииклов.

ПРЕДЛОЖЕНИЕ 9.7. Значение Arf-инварианта квадратичной формы совпадает с четностью числа особих точек $\Delta_{3}$ типа В для заданной локальной Prem-структуры.

ДОКАЗАТЕЛЬСТВО ЛЕММЫ 9.6 И ПРЕДЛОЖЕНИЯ 9.7. В СОоТветствИИ с разбИением $\bar{\Delta}=\bar{\Delta}_{+} \cup \bar{\Delta}_{-}$определено отображение $\tilde{f}: M^{2} \rightarrow R^{4}, \pi \circ \tilde{f}=f$, такое, что $\tilde{f}-$ вложение всюду, за исключением изолированных особых точек $\rho_{i} \in \mathbb{R}^{4}$, причем точки $\pi\left(\rho_{i}\right)$ совпадают с точками $\Delta_{3}$ типа В на поверхности $f\left(M^{2}\right)$. Для произвольной точки $y \in \Delta \backslash U\left(\Delta_{3}\right)$, не лежащей в малой окрестности множества $\Delta_{3}$, прообразы $\bar{y}_{1}, \bar{y}_{2} \in \bar{\Delta}$ упорядочены вдоль оси проекции $\pi$ так, что точка $\bar{y}_{2}$ с большей координатой расположена на кривой $\Delta_{+}$, а точка $\bar{y}_{1}$ с меньшей координатой расположена на ветви кривой $\Delta_{-}$. Обозначим через $\bar{U}_{i} \subset \mathbb{R}^{4}$ маленький четырехмерный шар с центром в точке $\rho_{i}$, внутри которого $\tilde{f}$ не является вложением. Шары $U_{i}, i=1, \ldots, k$, при этом взаимно однозначно соответствуют точкам $\Delta_{3}$. Определено замкнутое односвязное 4 -многообразие $K$, полученное специальной перестройкой многообразия $\mathbb{R}^{4} \cup\{\infty\} \backslash \bigcup_{i=1}^{k} \bar{U}_{i}$ вдоль границы. Коротко конструкция многообразия $K$ состоит в следующем, подробности см. [6], [13]. В результате перестройки к каждому 3 -шару $\partial \bar{U}_{i}$ приклеивается 4-многообразие $V_{i}, \partial V_{i}=S^{3}$, причем $H_{2}\left(V_{i}, \partial V_{i} ; \mathbb{Z}\right)=\mathbb{Z}^{3} \oplus \mathbb{Z}^{8}$, а симметрическая форма, определенная на этой группе гомологий по двойственности Пуанкаре, для точки типа $B_{+}$изоморфна $(+1)^{3} \oplus E_{8}$, а для точки типа $B_{-}$изоморфна $(-1)^{3} \oplus\left(-E_{8}\right)$. При этом многообразие $K^{4}$ удовлетворяет следуюшим свойствам.

1. Сигнатура $\sigma(K)$ многообразия $K^{4}$ вьгисляется по формуле:

$$
\sigma(K)=11\left(\operatorname{ord}\left(B_{+}\right)-\operatorname{ord}\left(B_{-}\right)\right) \text {. }
$$

2. Определено вложение $\alpha: M^{2} \rightarrow K^{4}$, при этом поверхность $\alpha \bar{f}(M)$ является характеристической (определение характеристической поверхности см. в [31]) и индекс 
самопересечения $\langle\alpha ; \alpha\rangle$ поверхности в многообразии $K^{4}$ вычисляется по формуле

$$
\langle\alpha ; \alpha\rangle=3\left(\operatorname{ord}\left(B_{+}\right)-\operatorname{ord}\left(B_{-}\right)\right)
$$

3. Квадратичная форма многообразия $K^{4}$, восстановленная по характеристической поверхности $\alpha(M)$, совпадает с квадратичной формой $q(f, O)$.

Свойство 3 и корректность определения квадратичной формы характеристической поверхности доказывает лемму 9.6.

По теореме Рохлина о сигнатуре, см. [71], [31], справедливо тождество:

$$
\operatorname{Arf}(q)=\frac{1}{8}(\sigma(K)-\langle\alpha, \alpha\rangle) \quad(\bmod 2)
$$

и, следовательно,

$$
\operatorname{Arf}(q)=\operatorname{ord}\left(B_{+}\right)-\operatorname{ord}\left(B_{-}\right) \quad(\bmod 2)
$$

Предложение 9.7 доказано.

Оказывается, что поверхность Константинова доставляет пример поверхности, для которой множество тройных точек типа В представлено единственной точкой при соответствуюшем выборе локальной Prem-структуры.

Пример 9.8. Рассмотрим поверхность Константинова $\varphi: T^{2} \rightarrow \mathbb{R}^{3}$, изображенную на рис. 5. Кривая $\Delta_{2}$ имеет 3 компоненты связности. Определим ориентацию $O$ на этой кривой. На одной из компонент определим ориентацию произвольньм образом и индуцируем ориентации двух оставшихся компонент посредством врашения на угол $2 \pi / 3$ вокруг оси, проходящей через центральную точку $\Delta_{3}$ перпендикулярно плоскости проекции. При этом центральная тройная точка будет иметь тип В, остальные три трехкратные точки будут иметь тип А. Для разновидностей $B_{+}, B_{-} ; A_{+}, A_{-}$ вьполнено тождество

$$
\operatorname{ord}\left(B_{+}\right)-\operatorname{ord}\left(B_{-}\right)+3 \operatorname{ord}\left(A_{+}\right)-3 \operatorname{ord}\left(A_{-}\right)=0
$$

В самом деле, равенство (25) вытекает из того факта, что ориентированньй 1-цикл $\Delta(f)$, приведенньй в общее положение с погруженной поверхностью, пересекает эту поверхность с алгебраической кратностью 0.

Разновидность центральной тройной точки зависит от выбора ориентации $O$. Если условиться о выборе ориентации инвариантной относительно поворота, как было указано вьше, то типы и разновидности трех трехкратных точек, отличных от центральной, должны совпадать из-за симметрии. Вычисление типов и разновидностей является легким упражнением на применение формулы (25), подробнее см. [4], [6].

Пусть $f: M^{2} \rightarrow \mathbb{R}^{3}$ - ориентированная поверхность общего положения, причем кривая $\Delta$ состоит из $s$ компонент. Определено $2^{s}$ попарно различных ориентаций $O$ на этой несвязной кривой. Для каждой из этих ориентаций определена квадратичная форма $q(f, O)$. 
АЛГЕБРАИЧЕСКОЕ ПРЕПЯТСТВИЕ К РЕШЕНИЮ ЗАДАЧИ РЕАЛИЗАЦИИ. Опpeделим алгебраическое препятствие (частичное) $к$ реализации отображения $I \circ f: M^{2} \rightarrow \mathbb{R}^{4}$ по формуле

$$
\beta(f)=\Pi_{j} \operatorname{Arf}\left(q\left(f, O_{j}\right)\right)(\bmod 2),
$$

где $O_{j}$ пробегает мнохество всех ориентаций, определяющих попарно различные локальные Prem-структуры.

Важно отметить, что препятствие $\beta$ определяется по отображению $f$ и не зависит от выбора локальной Prem-структуры.

В работе [13] рассматривается модификация поверхности Константинова $\psi \rightarrow \varphi$ такая, что $\beta(\varphi)=1$.

ЗАмЕчАниЕ. Сходная алгебраическая конструкция для погруженных многообразий старших размерностей $4 k+2$ имеется в работе Джонса и Рииса [42]. Она лежит в основе доказательства знаменитой теоремы Браудера о стабильно-параллелизуемых многообразиях с инвариантом Кервера 1.

В работе [49] решается задача о построении вложения в данном классе гомотопии $g: M^{2} \rightarrow N^{4}$ замкнутой ориентированной поверхности в замкнутое ориентированное 4-многообразие. Используется теория Дональдсона, и определяется алгебраическое целочисленное препятствие к решению этой задачи. Естественно поставить вопрос о нахождении целочисленного алгебраического аналога препятствия $\beta(f)$. Конструкция этого аналога приводит к интерпретации главного инварианта Васильева $V_{2}$ узлов, см. [85], как аналога инварианта странности плоских кривых, определенных для диаграмм узлов. Напомним определение инварианта странности плоской кривой, построенного В.И. Арнольдом в работе [15].

Каждой кривой $\varphi: S^{1} \rightarrow \mathbb{R}^{2}$ общего положения сопоставляется число $\operatorname{St}(\varphi)$, называемое инвариантом странности кривой $\varphi$, которое удовлетворяет следующему свойству (и определяется этим свойством). Пусть $\varphi_{t}, t \in\left[t_{0}-\varepsilon ; t_{0}+\varepsilon\right],-$ деформация кривой общего положения в классе погружений, так что при $t=t_{0}$ погружение $\varphi_{t_{0}}$ имеет тройную точку самопересечения $y$. В окрестности точки $y$ при $t=t_{0}-\varepsilon$ возникает “исчезающий треугольник” и при $t=t_{0}+\varepsilon$ рождается другой треугольник. Стороны "исчезающего треугольника" снабжены циклическим порядком, согласованным с порядком обхода вдоль кривой $\varphi\left(S^{1}\right)$ в положительном направлении. Этот порядок определяет ориентацию $O^{\prime}$ сторон “исчезающего треугольника". Пусть $q$ - число согласований ориентации $O^{\prime}$ со стандартной ориентацией $O$ сторон треугольника, индуцированной ориентацией на окружности. Значения $\operatorname{St}\left(\varphi_{t_{0}-\varepsilon}\right)$ и $\operatorname{St}\left(\varphi_{t_{0}+\varepsilon}\right)$ инвариантов странности связаны по формуле:

$$
\operatorname{St}\left(\varphi_{t_{0}-\varepsilon}\right)-\operatorname{St}\left(\varphi_{t_{0}+\varepsilon}\right)=(-1)^{q}
$$

ЗАмечАниЕ. Обозначение St для инварианта странности предложено В.И. Apнольдом в [15]. Такое же общепринятое обозначение использовалось для групшы Стейнберга, см. раздел 8. Установилась также неоднозначность, связанная с понятием псевдоизотопии. Первоначально этот термин употреблялся в геометрической топологии в том же смысле, как в первой части. После работы Серфа [17] стал 
употребляться в ином смысле, как в ч. 2. К сожалению, неоднозначности избежать здесь не удается, поскольку обозначение и термин являются в каждом контексте общепринятыми.

Поскольку произвольная погруженная в плоскость кривая связана регулярной гомотопией со стандартной кривой в данном классе регулярной гомотопии (класс регулярной гомотопии определяется индексом Уитни), формула (26) определяет значение инварианта странности для любой погруженной кривой, если задано значение инварианта на стандартной. Инвариант странности не изменяется при регулярных гомотопиях кривых без особенностей троекратного самопересечения. Нас далее будет интересовать случай индекса Уитни +1 , в этом случае класс регулярной гомотопии погруженной кривой представлен стандартной вложенной окружностью, ориентированной против часовой стрелки. На этой кривой значение инварианта St по определению равно 0. Наша задача интерпретировать инвариант Васильева $V_{2}$ узлов в $\mathbb{R}^{3}$ (см. [85]) как обобщенный инвариант странности для диаграмм проекций узлов на плоскость.

Под диаграммой узла, которую мы будем называть Prem-орнаментом (точнее, невырожденньм Рrem-орнаментом), будем понимать иммерсированную кривую $\varphi: S^{1} \rightarrow \mathbb{R}^{2}$ обшего положения вместе с функцией $O: \Delta(\varphi) \rightarrow\{+1,-1\}$, определенной на конечном множестве точек двукратного самопересечения. Понятие Prem-орнамента отличается от понятия проекции узла, так как уже в простейших конструкциях приходится рассматривать Prem-орнаменты более обшего вида, не соответствующие проекции никакого узла, которые встречаются в однопараметрических семействах общего положения. Произвольньй невырожденньй Prem-орнамент в точности соответствует проекции узла $\pi \circ \varphi: S^{1} \rightarrow \mathbb{R}^{3} \rightarrow \mathbb{R}^{2}$, при этом в точке $x_{i} \in \Delta_{2}(\varphi)$ значение функции определяется следуюшим образом. Рассмотрим базис $\xi_{1}, \xi_{2}$ на плоскости, образованньй касательньми векторами к ветвям кривой $\varphi\left(S^{1}\right)$, причем порядок векторов базиса соответствует значению координаты ветвей кривой вдоль оси проекции. Условимся, что первый вектор имеет бо́льшую координату. Значение функции $O$ определим как +1 , если базис положительно ориентирован в плоскости, и -1 в другом случае. Скажем, что Prem-орнамент является нескрученным, если индекс Уитни кривой $\varphi$ равен +1 и вьполнено тождество

$$
\sum_{i} O\left(x_{i}\right)=0, \quad x_{i} \in \Delta_{2}(\varphi)
$$

Рассмотрим следующие три преобразования Prem-орнаментов, представленные на рис. 7 в малой окрестности особых точек и неподвижные вне этих окрестностей.

Преобразования 1,2 соответствуют проекциям изотопии узлов в пространстве и назьваются преобразованиями Райдемайстера диаграмм узла. Преобразование 3 не соответствует никакой изотопии. Это преобразование будем назьвать $\delta$-движением, следуя построениям Мураками и Наканиши [60]. (См. также работу С. В. Матвеева [55], в которой аналогичные построения были проведены в частном случае.)

ПРЕДЛОЖЕНИЕ 9.9. Два произвольных нескрученных $\operatorname{Prem-орнамента~}\left(\varphi_{1}, O_{1}\right)$, $\left(\varphi_{2}, \mathrm{O}_{2}\right)$ связана между собой регулярными деформациями и преобразованиями 1-3, puc. 7 . 

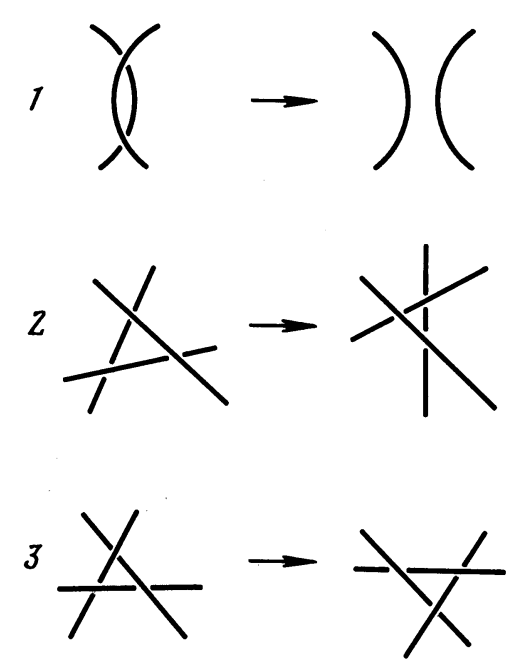

Рис. 7

Следующее предложение дает ответ на изучаемую проблему.

ПРЕДЛОЖЕНИЕ 9.10. На пространстве Prem-орнаментов определен инвариант $V_{2}$ преобразований Райдемайстера Prem-орнаментов. При обобщенном преобразовании Райдемайстера значение инварианта меняется в соответствии $c$ формулой:

$$
V_{2}\left(t_{0}+\varepsilon\right)-V_{2}\left(t_{0}-\varepsilon\right)=\operatorname{St}\left(\varphi_{t_{0}+\varepsilon}\right)-\operatorname{St}\left(\varphi_{t_{0}-\varepsilon}\right)
$$

где $\mathrm{St}$ - инвариант странности погруженной кривой, $x_{i}, i=1,2,3,-$ вериинь исчезающего треугольника.

ДокАЗАТЕЛЬСТво. Предложение вытекает из результатов работы О. Виро и М. Поляка [63], оно изложено в [7].

ПроБЛЕмА. Интерпретировать инварианты Васильева стариих порядков в терминах аналогов высших инвариантов странности Prem-орнаментов с учетом особенностей $n$-кратного самопересечения при $(n-1)$-параметрических деформациях.

ЗАмЕчАнИЕ. Решение проблемы для узлов при $n<5$ вытекает из результатов работ [63], [83]. Проблема особенно интересна для случая ориентированных многокомпонентных зацеплений. В этом случае проблему можно уточнить: можно ли частично определенные инварианты конечных порядков многокомпонентных зацеплений естественным образом доопределить до однозначных? Под естественностью понимается сохранение порядка инварианта. С проблемой доопределения частичных инвариантов Масси зацеплений до полных связаны результаты работы [72]. 


\section{СПИСОК ЛИТЕРАТУРЫ}

[1] Adachi M. Embeddings and Immersions. Providence, RI: Amer. Math. Soc., 1993.

[2] Adams J.F. The Kahn-Priddy theorem // Proc. Cambridge Philos. Soc. 1973. V. 73. P. 43-55.

[3] Adams J. F. On the non-existence of elements of Hopf-invariant one // Ann. of Math. (2). 1960. V. 72. P. 2-104.

[4] Ахметьев П. М. Гладкие погружения многообразий мальх размерностей. I // Матем. сб. 1994. Т. 185. №10. С. 3-23.

[5] Ахметьев П.М. Гладкие погружения многообразий малых размерностей. II. Группы кобордизма критических значений многопараметрических семейств функций // Матем. сб. 1995. Т. 186. № 12. C. 37-62.

[6] Ахметьев П. М. Рrem-отображения, тройные точки ориентированной поверхности и теорема Рохлина о сигнатуре // Матем. заметки. 1996. Т. 59. №6. С. 803-810.

[7] Akhmet'ev P. M. On a higher analog of the linking number of two curves // Amer. Math. Soc. Transl. Ser. 2. 1998. V. 185. P. 113-127.

[8] Ахметьев П. М. Об изотопической и дискретной реализации отображений $n$-сферы в евклидовом пространстве // Матем. сб. 1996. Т. 187. № 7. С. 3-34.

[9] Ахметьев П. М. Реализация отображений $n$-сферы в евклидовом $2 n$-пространстве // Труды МИАН. 1996. Т. 212. С. 37-45.

[10] Ахметьев П. М. Обобщенная проблема Давермана // Труды Международной математической конференции к 90-летию Л. С. Понтрягина. Т. 87. М.: ВИНИТИ, 2000. С. 1-11.

[11] Ахметьев П. М., Мелихов С. А. Об изотопической реализуемости непрерывных отображений // Уч. зап. науч. семин. ПОМИ (в печати).

[12] Akhmet'ev P. M., Szucs A. On the Hopf invariant in homotopy groups of spheres, geometric approach // Math. Slovaca. 1999. V. 49. P. 71-74.

[13] Akhmet'ev P.M., Repovs D., Skopenkov A.B. Obstruction to approximating maps of surfaces into $\mathbb{R}^{4}$ by embeddings // Preprint. Ljubljana: Ljubljana Univ., 1998; Topology Appl. (to appear).

[14] Александров П. С. О размерности замкнутых множеств // УМН. 1949. T. IV. №6. C. $17-88$.

[15] Arnol'd V. I. Topological Invariants of Plane Curves and Caustics. Providence, RI: Amer. Math. Soc., 1994.

[16] А рнольд В.И.О пространствах функций с умеренными особенностями // Функц. анализ и его прил. 1989. Т. 23. № 3. С. 1-10.

[17] Cerf J. La stratification naturelle des espaces de fonctions différentiables réeles et le théorème de la pseudo-isotopie // Inst. Hautes Études Sci. Publ. Math. 1970. V. 39. P. 5-173.

[18] Чернавский А. В. Локалная стягиваемость группы диффеоморфизмов многообразия // Матем. сб. 1969. Т. 79. №3. С. 307-356.

[19] Chess D. Singularity theory and configuration space models of $\Omega^{n} S^{n}$ of non-connected spaces // Topology Appl. 1987. V. 25. P. 313-338

[20] Cohen R. L. The immersion conjecture for differentiable manifolds // Ann. of Math. (2). 1985. V. 122. P. 237-328.

[21] Daverman R. J. Problems about finite-dimensional manifolds // Open Problems in Topology / ed. J. van Mill, G. M. Reed. Amsterdam: North-Holland, 1990.

[22] Dranishnikov A. N., Repovs D., Scepin E. V. On the intersection of compacta of complementary dimension in Euclidean space // Topology Appl. 1991. V. 38. P. 237-253.

[23] Дубровин Б. А., Новиков С. П., Фоменко А. Т. Современная геометрия. Методы теории гомологий. М.: Наука, 1982.

[24] Eccles P. J. Codimension one immersions and the Kervaire invariant one problem // Math. Proc. Cambridge Philos. Soc. 1981. V. 90. P. 483-493.

[25] Eliashberg Ya., Mishachev N. M. Wrinkling of smooth mappings and its applications. I // Invent. Math. 1997. V. 130. P. 345-369. 
[26] Fenn R. Some generalizations of the Borsuk-Ulam theorem and applications to realizing homotopy classes by embedded spheres // Proc. Cambridge Philos. Soc. 1973. V. 74. P. 251-256.

[27] Фрэнсис Дж. К. A topological Picturebook. Berlin: Springer-Verlag, 1987; Перевод: Книжка с картинками по топологии. М.: Мир, 1991.

[28] Freedman M. Quadruple points of 3-manifolds in $\mathbb{R}^{4} / /$ Comment. Math. Helv. 1978. V. 53. P. 385-394.

[29] Giller C. Towards a classical knot theory for surfaces in $\mathbb{R}^{4} / /$ Illinois J. Math. 1982. V. 26. P. 591-631.

[30] Громов М. Дифференциальные соотношения с частными производными. М.: Мир, 1990.

[31] Гийу Л., Марэн А. В поисках утраченной топологии. М.: Мир, 1986.

[32] Hatcher A. Concordance spaces, higher simple-homotopy theory, and applications // Proc. Sympos. Pure Math. 1978. V. 32. P. 3-21.

[33] Hatcher A. E., Wagoner J. B. Pseudo-isotopies of compact manifolds. I. The first obstruction for pseudo-isotopies // Astérisque. 1973. №6. P. 8-238.

[34] Heafliger A. Plongements différentiables dans le domaine stable // Comment. Math. Helv. 1962-1963. V. 37. P. 155-176.

[35] Hirsh M. W. Immersions of manifolds // Trans. Amer. Math. Soc. 1960. V. 93. P. 242-276.

[36] Hughes B., Ranicki A. Ends of Complexes. Cambridge: Cambridge Univ. Press, 1996. (Cambridge Tracts in Math. V. 123.)

[37] Гуревич В., Волмэн Г. Теория размерности. М.: ГИИЛ, 1948.

[38] Hush L.S. $\varepsilon$-maps and embeddings // General Topology and its Relations to Modern Analysis and Algebra. VI. Proceedings of the Sixth Prague Topological Symposium, 1986 / ed. Z. Frolik. Berlin: Heldermann-Verlag, 1988. P. 237-280.

[39] Igusa K. On the homotopy type of the space of generalized Morse functions // Topology. 1984. V. 23. № 2. P. 245-256.

[40] Igusa K. The space of framed functions // Trans. Amer. Math. Soc. 1987. V. 301. № 2 . P. 431-477.

[41] James I. On the iterated suspension // Quart. J. Math. Oxford Ser. (2). 1954. V. 5. P. 1-10.

[42] Jones J., Rees E. Kervaire's invariant for framed manifolds // Proc. Sympos. Pure Math. 1978. V. 32. P. 141-147.

[43] Казарян М.Э. Скрытые особенности и гомологический комплекс Василева классов особенностей // Матем. сб. 1995. Т. 186. № 12. С. 119-128.

[44] Келдыш Л. В. Топологические вложения в евклидово пространство // Труды МИАН. 1966. T. 81 . C. $3-183$.

[45] Keesling J., Wilson D. C. Embedding $T^{n}$-like continua in Euclidean space // Topology Appl. 1985. V. 21. P. 241-249.

[46] Koschorke U. Multiple points of immersions and the Kahn-Priddy theorem // Math. Z. 1979. V. 169. P. 223-236.

[47] Koschorke U., Sanderson B. Geometric interpretations of the generalized Hopf invariant // Math. Scand. 1977. V. 41. P. 199-217.

[48] Krasinkewicz J. Imbeddings into $\mathbb{R}^{n}$ and dimension of products // Fund. Math. 1986. V. 133. P. 247-253.

[49] Kronheimer P. B. An obstruction to removing intersection points in immersed surfaces // Topology. 1997. V. 36. № 4. P. 931-962.

[50] Lannes J. Sur les immersions de Boy // Lecture Notes in Math. 1984. V. 1051. P. 263-270.

[51] Laudenbach F. Formes différentielles de degrée 1 non singuliéres: classes d'homotopie de leurs noyaux // Comment. Math. Helv. 1976. V. 51. P. 447-464.

[52] Levine H. Classifying immersions into $\mathbb{R}^{4}$ over stable maps of 3-manifolds into $\mathbb{R}^{2}$. Berlin: Springer-Verlag, 1985. (Lecture Notes in Math. V. 1157.)

[53] Liulevicius A. A theorem in homological algebra and stable homotopy of projective spaces // Trans. Amer. Math. Soc. 1963. V. 109. P. 540-552.

[54] Loffler P., Ray N. A Geometrical proof of a theorem of Kahn and Priddy // J. London Math. Soc. (2). 1981. V. 24. P. 329-334. 
[55] Матвеев С. В. Обобщенные перестройки трехмерных многообразий и представления гомологических сфер // Матем. заметки. 1987. Т. 42. № 2. С. 268-278.

[56] Melikhov S. On maps with unstable singularities // Topology Appl. (to appear).

[57] Milnor J. W. Введение в алгебраическую $K$-теорию. М.: Мир, 1974.

[58] Мишачев Н. М. Отображения с простейшими особенностями // Добавление в книге Громова М. Дифференциальные соотношения с частными производными. М.: Мир, 1990.

[59] Мошер Р. С., Тангора М. Когомологические операции и их применение в теории гомотопий. М.: Мир, 1970.

[60] Murakami H., Nakanishi Y. On a certain move generating link-homology // Math. Ann. 1989. V. 284. P. 75-89.

[61] Nöbeling G. Über eine $n$-dimensionale Universalmenge im $R_{2 n+1} / /$ Math. Ann. 1930 . V. 104. P. $71-80$.

[62] Пуанкаре А. О науке. М.: Наука, 1983.

[63] Polyak M., Viro O. Gauss diagram formulas for Vassiliev invariants // Internat. Math. Res. Notices. 1994. № 11. P. 445-453.

[64] Pontryagin L.S. Sur une hypothése fondamentale de la théorie de la dimension // C. R. Acad. Sci. Paris. 1930. V. 190. P. 1105-1107.

[65] Понтрягин Л. С. Гладкие многообразия и их применение в теории гомотопий. М.: Наука, 1976.

[66] Постников М. М. Группы и алгебры Ли. М.: Наука, 1982.

[67] Quinn F. Local algebraic topology // Notices Amer. Math. Soc. 1981. V. 11. P. 895-899.

[68] Repovš D., Skopenkov A. B. Embeddability and isotopy of polyhedra in Euclidean spaces // Труды МИАН. 1996. Т. 212. С. 173-187.

[69] Repovs D., Skopenkov A. B. A deleted product criterion for approximability of maps by embeddings // Topology Appl. 1998. V. 87. P. 1-19.

[70] Реповш Д., Скопенков А. Б. Кольца Борромео и препятствия к вложимости // Труды МИАН. 1999. Т. 225. С. 331-338.

[71] Рохлин В. А. Доказательство гипотезы Гудкова // Функц. анализ и его прил. 1972. Т. 6. № 2. C. $62-64$.

[72] Rybnikov G. On the fundamental group and triple Massey's product // Препринт. М.: Московский Независимый Университет, 1998.

[73] Шепин Е. В., Штанько М. А. Спектральный критерий для вложения компактов в евклидовы пространства // Труды Ленинградской топологической международной конференции, Т. II. М.: Наука, 1983. С. 135-142.

[74] Штанько М.А. Вложение компактов в евклидово пространство // Матем. сб. 1970. T. 83. № 2. C. 234-255.

[75] Sieklucki K. The realization of mappings // Fund. Math. 1969. V. 65. P. 325-343.

[76] Sikkema C. P. Pseudo-isotopies of arcs and knots // Proc. Amer. Math. Soc. 1972. V. 31. № 2. P. $615-616$.

[77] Skopenkov A. On the deleted product criterion for embeddability in $\mathbb{R}^{m} / /$ Proc. Amer. Math. Soc. 1998. V. 126. № 8. P. 2467-2476.

[78] Smale S. The classification of immersions of spheres in Euclidean spaces // Ann. of Math. (2). 1959. V. 69. P. 327-334.

[79] Spiez S. On pairs of compacta with $\operatorname{dim}(X \times Y)<\operatorname{dim}(X)+\operatorname{dim}(Y) / /$ Fund. Math. 1990 . V. 135. P. 213-222.

[80] Szucs A. Geometrical proof of a theorem on suspension in homotopy groups of spheres // Colloq. Math. Soc. János Bolyai (Hungary). 1989. V. 55. P. 501-505.

[81] Szucs A. Cobordism groups of immersions of oriented manifolds // Acta Math. Hungar. 1994. V. 64. № 2. P. 191-230.

[82] Тода Х. Композиционные методы в теории гомотопических групп сфер. М.: Наука, 1982.

[83] Тюрина С. Д. Диаграммные формулы типа Виро-Поляка для инвариантов конечного порядка // УМН. 1998. Т. 54. № 3. 
[84] Vassiliev V.A. Lagrange and Legendre Characteristic Classes. New Yourk: Gordon \& Breach, 1993; русский перевод: Васильев В.А. Лагранжевы и лежандровы характеристические классы. М.: МЦНМО, 1999.

[85] Васильев В. А. Топология дополнений к дискриминантам. М.: Фазис, 1997.

[86] Васильев В.А. Топология пространств функций без сложных особенностей // Функц. анализ и его прил. 1989. Т. 13. № 4. С. 24-36.

[87] Weber C. Plongements de polyèdres dans le domaine métastable // Comment. Math. Helv. 1967. V. 42. P. 1-27.

[88] Wells R. Cobordism groups of immersions // Topology. 1966. V. 5. P. 281-294.

Математический институт им. В. А. Стеклова РАН

Поступила в редакцию

E-mail: pmakhmet@mi.ras.ru 